\title{
Synthesis of functionalized furans via chemoselective reduction/Wittig reaction using catalytic triethylamine and phosphine
}

Chia-Jui Lee, Tzu-Hsiu Chang, Jhen-Kuei Yu, Ganapuram Madhusudhan Reddy, Ming-Yu Hsiao and Wenwei Lin*

Department of Chemistry, National Taiwan Normal University, 88, Sec. 4, Tingchow Road, Taipei 11677, Taiwan, R.O.C.

wenweilin@ntnu.edu.tw

Table of Contents:

I. General information S2-S2

II. Mechanism study : Control experiments related to the reduction of phosphine oxide 3

S3-S4

III. Typical experimental procedures

S4-S5

IV. Analytical data for the products

S6-S34

V. References

S34-S34

VI. ${ }^{1} \mathrm{H}$ NMR and ${ }^{13} \mathrm{C}$ NMR spectra of the products

S35-S106 


\section{General information:}

All reactions were carried out under a argon atmosphere in dried Schlenk flask. The starting materials purchased from commercial sources were used without further purification. Acetonitrile were continuously refluxed and freshly distilled from potassium hydroxide under nitrogen. IR spectra were recorded on a Perkin Elmer 500 spectrometer. NMR spectra were recorded on a Bruker Avance 400/500 NMR spectrometer. Chemical shifts are reported in $\delta$ ppm referenced to an internal TMS standard for ${ }^{1} \mathrm{H}$ NMR and chloroform-d ( $\left.\delta 77.0 \mathrm{ppm}\right)$ for ${ }^{13} \mathrm{C}$ NMR. HRMS spectra were recorded on JEOL SX-102A. The X-ray diffraction measurements were carried out at $298 \mathrm{~K}$ on a KAPPA APEX II CCD area detector system equipped with a graphite monochromator and a Mo-Ka fine-focus sealed tube $(\mathrm{k}=0.71073 \AA$ A). Analytical thin layer chromatography (TLC) was performed using Merck 60 F254 precoated silica gel plate $(0.2 \mathrm{~mm}$ thickness $)$. Flash-chromatography was performed using Merck silica gel 60 (70-230 mesh). 
II. Mechanism study: Control experiments related to the reduction of phosphine oxide 3.

(1) A series of control experiments that monitored the reduction process at $30{ }^{\circ} \mathrm{C}$ by ${ }^{31} \mathbf{P}$ NMR

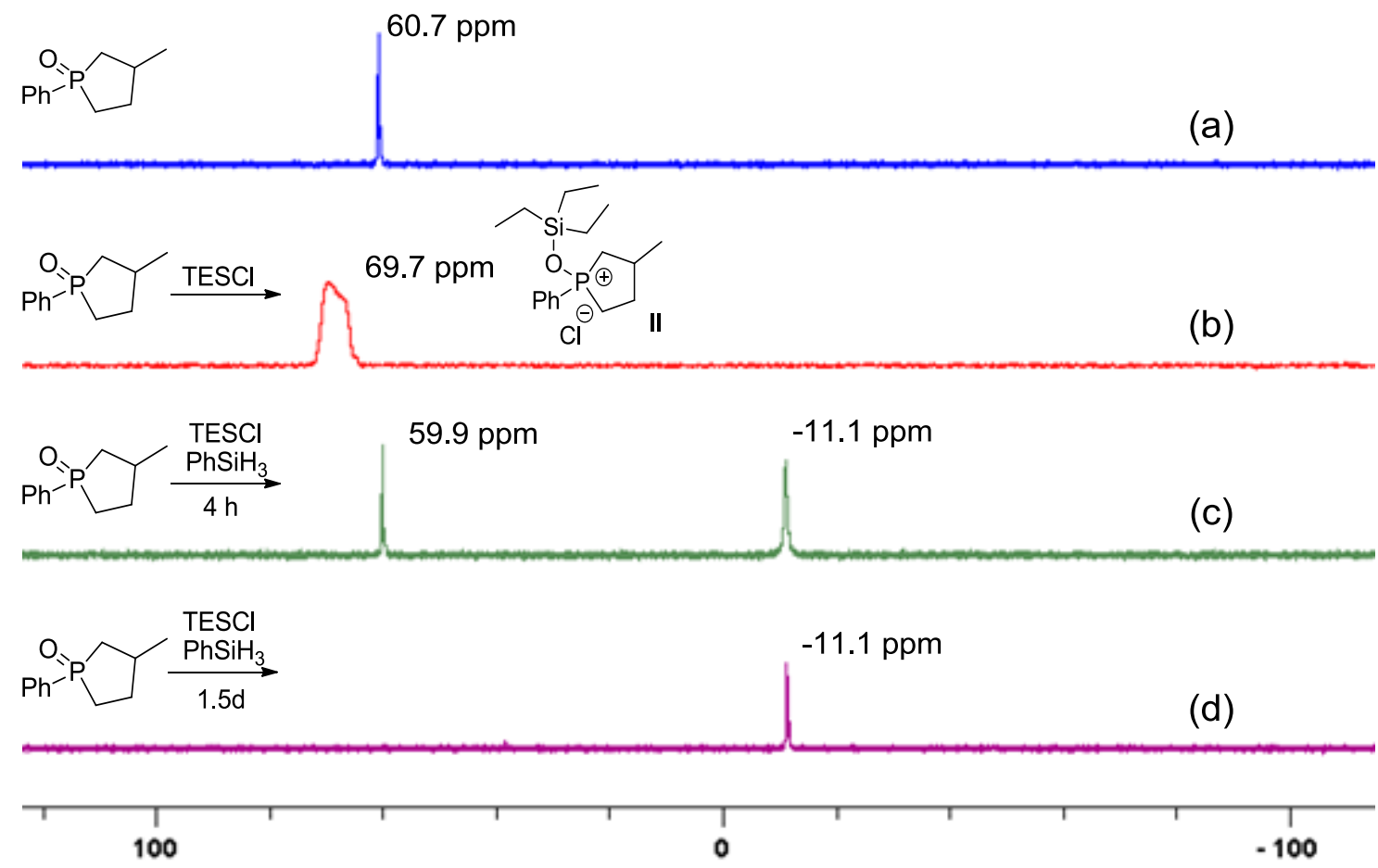

A dry and argon-flushed NMR tube equipped with a septum was charged with 3 (0.2 mmol) and $\mathrm{CDCl}_{3}(1 \mathrm{~mL})$ at $30{ }^{\circ} \mathrm{C}$. It was monitored by ${ }^{31} \mathrm{P}$ NMR (plot-a).

First of all, TESCl ( 2 equiv.) was added to the reaction mixture and the reaction progress was monitored by ${ }^{31} \mathrm{P}$ NMR (plot-b).

Then $\mathrm{PhSiH}_{3}$ (16 equiv) was added into the same reaction mixture. Four hour later ${ }^{31} \mathrm{P}$ NMR (plot-c) was recorded.

Finally, the reaction was prolonged for 1.5 days and ${ }^{31} \mathrm{P}$ NMR was recorded again (plot-d).

While phosphine oxide 3 was treated with TESCl, appearance of a broad peak at 69.7 ppm was seen (plot-b). We assume this might be a characteristic peak of intermediate II. Furthermore, when $\mathrm{PhSiH}_{3}$ was added to this reaction mixture, two peaks located at 59.9 and $-11.1 \mathrm{ppm}$ were seen in the ${ }^{31} \mathrm{P}$ NMR (plot-c). After 1.5 days, it was observed that all the phosphine oxide $\mathbf{3}$ was reduced (plot-d). 
(2) Control experiments to demonstrate the influence of TESCl towards the reduction of phosphine oxide 3 at $30{ }^{\circ} \mathrm{C}$.
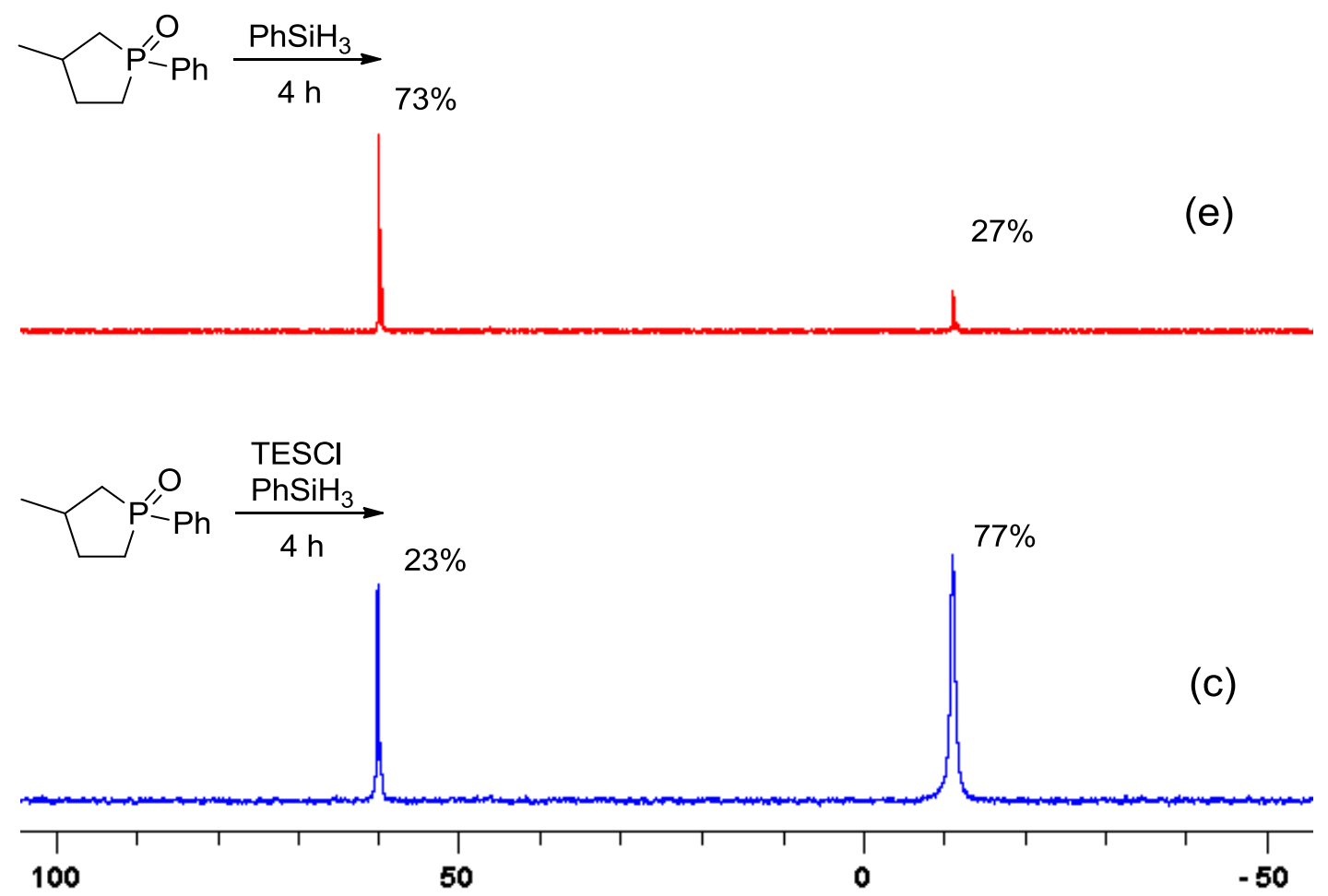

Two dry and argon-flushed NMR tubes equipped with septums were charged with $\mathbf{3}$ $(0.2 \mathrm{mmol})$ and $\mathrm{CDCl}_{3}(1 \mathrm{~mL})$ at $30{ }^{\circ} \mathrm{C}$. The first tube was treated with $\mathrm{PhSiH}_{3}(16$ equiv), while the second was was treated with TESCl (2 equiv.) and $\mathrm{PhSiH}_{3}$ (16 equiv). Four hour later, the progress of both the reactions was monitored by ${ }^{31} \mathrm{P} N \mathrm{NR}$.

From the result, we could conclude that TESCl indeed assists in the reduction process.

\section{Typical experimental procedures}

\section{TP-A (Tables 2-4 and Scheme 2):}

A dry and argon-flushed $10 \mathrm{~mL}$ Schlenk flask equipped with a magnetic stirring bar and a septum was sequentially charged with $\mathbf{1}(0.3 \mathrm{mmol})$, dry THF $(1.5 \mathrm{~mL}), \mathbf{3}(10$ mol \%), $\mathrm{Et}_{3} \mathrm{~N}$ (20 mol \%), phenylsilane (1.6 equiv), TESCl (4b) (20 mol \%) and acyl chloride 2 (1.1 equiv). The reaction mixture was stirred for corresponding time at 50 ${ }^{\circ} \mathrm{C}$. Then the solvent was removed by evaporation in vacuo and the crude mixture was purified by flash column chromatography to provide $\mathbf{5}$. 


\section{TP-B (Table 5):}

A dry and argon-flushed $10 \mathrm{~mL}$ Schlenk flask equipped with a magnetic stirring bar and a septum was sequentially charged with dry THF $(1.5 \mathrm{~mL}), 3(10 \mathrm{~mol} \%)$, phenylsilane (1.6 equiv) and with the corresponding reagents in table 5 . The reaction mixture was stirred for 4 hours at corresponding temperature and directly diluted with $\mathrm{CDCl}_{3}$ for the NMR experiments to determine the reduction of $\mathbf{3}$.

\section{TP-C (Table 6):}

A dry and argon-flushed $10 \mathrm{~mL}$ Schlenk flask equipped with a magnetic stirring bar and a septum was sequentially charged with dry THF $(1.5 \mathrm{~mL}), 3$ or tributylphoshpine oxide (10 mol \%), phenylsilane (1.8 equiv) and 2a (1.1 equiv) with the corresponding reagents in table 6 . The reaction mixture was stirred for 4 hours at $50{ }^{\circ} \mathrm{C}$ and directly diluted by $\mathrm{CDCl}_{3}$ for the NMR experiments to determine the reduction of $\mathbf{2 a}$.

\section{TP-D (Scheme 3):}

A dry and argon-flushed $10 \mathrm{~mL}$ Schlenk flask equipped with a magnetic stirring bar and a septum was sequentially charged with $1 \mathrm{a}(0.3 \mathrm{mmol})$, dry THF $(1.5 \mathrm{~mL})$, tributylphosphine oxide (10 mol \%), $\mathrm{Et}_{3} \mathrm{~N}$ (20 mol \%), phenylsilane (1.8 equiv), TESCl (4b) (20 mol \%). The reaction mixture was stirred for 24 hours at $50{ }^{\circ} \mathrm{C}$. Then the solvent was removed by evaporation in vacuo and crude mixture was purified by flash column chromatography to provide $\mathbf{1 1}$. 


\section{Analytical data for the products}

Synthesis of 4-(2-bromophenyl)-5-(4-chlorophenyl)-2-phenylfuran-3-carbonitrile $(5 \mathbf{a a})^{[\mathrm{a}]}$

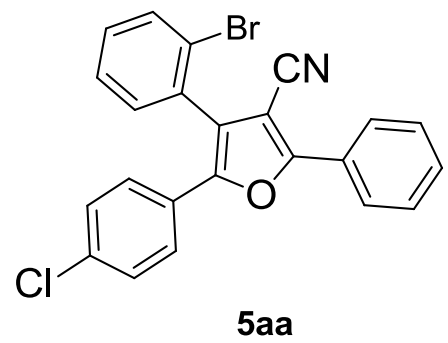

Prepared according to TP-A using $\mathbf{1 a}(93.6 \mathrm{mg}, 0.3 \mathrm{mmol}), 3(5.8 \mathrm{mg}, 0.03 \mathrm{mmol})$, $\mathrm{Et}_{3} \mathrm{~N}(8.4 \mu \mathrm{L}, 0.06 \mathrm{mmol}), \mathrm{PhSiH}_{3}(61 \mu \mathrm{L}, 0.48 \mathrm{mmol}), \mathbf{4 b}(10 \mu \mathrm{L}, 0.06 \mathrm{mmol}), \mathbf{2 a}$ $(42 \mu \mathrm{L}, 0.33 \mathrm{mmol})$ and THF $(1.5 \mathrm{~mL})$. The reaction mixture was stirred for 5 hours at $50{ }^{\circ} \mathrm{C}$. Then the solvent was removed by evaporation in vacuo, and crude mixture was purified by flash column chromatography (DCM:Hexanes $=1: 5)$ to provide 5aa as a white solid in $84 \%$ yield (109.1 mg)

mp.: 161.5-162.3 ${ }^{\circ} \mathrm{C}, \mathrm{R}_{f}=0.111 .(\mathrm{DCM}:$ Hexanes $=1: 5)$

${ }^{1} \mathbf{H}$ NMR $\left(400 \mathrm{MHz}, \mathrm{CDCl}_{3}, 25{ }^{\circ} \mathrm{C}\right) \delta / \mathrm{ppm}: 8.11(\mathrm{~d}, 2 \mathrm{H}, J=7.1 \mathrm{~Hz}), 7.77(\mathrm{~d}, 1 \mathrm{H}, J=$ 8.0 Hz), 7.57-7.42 (m, 4H), 7.41-7.33 (m, 4H), $7.28(\mathrm{~d}, 2 \mathrm{H}, J=8.8 \mathrm{~Hz})$

${ }^{13} \mathrm{C}$ NMR $\left(100 \mathrm{MHz}, \mathrm{CDCl}_{3}, 25{ }^{\circ} \mathrm{C}\right) \delta / \mathrm{ppm}: 158.1,148.0,134.7,133.6,131.8,131.3$, $130.9,130.4,129.2,129.0,128.2,127.7,127.4,126.5,125.5,124.4,123.5,113.9$, 96.3.

IR $(\mathrm{KBr}) \tilde{v}\left(\mathrm{~cm}^{-1}\right): 2232,1489,1099,730$.

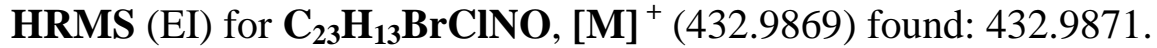

Synthesis of 4-(2-bromophenyl)-5-(4-chlorophenyl)-2-phenylfuran-3-carbonitrile (5ab)

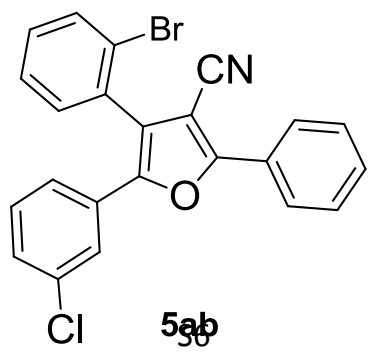


Prepared according to TP-A using 1a $(93.6 \mathrm{mg}, 0.3 \mathrm{mmol}), 3(5.8 \mathrm{mg}, 0.03 \mathrm{mmol})$, $\mathrm{Et}_{3} \mathrm{~N}(8.4 \mu \mathrm{L}, 0.06 \mathrm{mmol}), \mathrm{PhSiH}_{3}(61 \mu \mathrm{L}, 0.48 \mathrm{mmol}), \mathbf{4 b}(10 \mu \mathrm{L}, 0.06 \mathrm{mmol}), \mathbf{2 b}$ (43 $\mu \mathrm{L}, 0.33 \mathrm{mmol})$ and THF $(1.5 \mathrm{~mL})$. The reaction mixture was stirred for 6 hours at $50{ }^{\circ} \mathrm{C}$. Then the solvent was removed by evaporation in vacuo, and crude mixture was purified by flash column chromatography (DCM:Hexanes = 1:4) to provide 5ab as a white solid in $73 \%$ yield $(95.2 \mathrm{mg})$

mp.: $146.9-147.1{ }^{\circ} \mathrm{C}, \mathrm{R}_{f}=0.190 .(\mathrm{DCM}:$ Hexanes $=1: 4)$

${ }^{1} \mathbf{H}$ NMR $\left(400 \mathrm{MHz}, \mathrm{CDCl}_{3}, 25{ }^{\circ} \mathrm{C}\right) \delta / \mathrm{ppm}: 8.13(\mathrm{~d}, 2 \mathrm{H}, J=9.8 \mathrm{~Hz}), 7.78(\mathrm{~d}, 1 \mathrm{H}, J=$ $8.8 \mathrm{~Hz}), 7.54(\mathrm{t}, 2 \mathrm{H}, J=7.1 \mathrm{~Hz}), 7.52-7.41(\mathrm{~m}, 3 \mathrm{H}), 7.42-7.35(\mathrm{~m}, 2 \mathrm{H}), 7.28-7.25(\mathrm{~m}$, 1H), 7.21-7.18 (m, 2H).

${ }^{13} \mathrm{C}$ NMR (100 MHz, $\left.\mathrm{CDCl}_{3}, 25{ }^{\circ} \mathrm{C}\right) \delta / \mathrm{ppm}: 158.4,147.5,134.9,133.7,131.8,131.2$, $131.0,130.6,130.5,130.0,129.2,128.7,128.2,127.7,125.6,125.3,124.4,124.2$, 123.3, 113.8, 96.4.

IR $(\mathrm{KBr}) \tilde{v}\left(\mathrm{~cm}^{-1}\right): 2229,1491,767,688$.

HRMS (EI) for $\mathbf{C}_{\mathbf{2 3}} \mathbf{H}_{\mathbf{1 3}} \mathbf{B r C I N O}[\mathbf{M}]^{+}$(432.9869) found 432.9868 .

Synthesis of 4-(2-bromophenyl)-5-(2-chlorophenyl)-2-phenylfuran-3-carbonitrile (5ac)

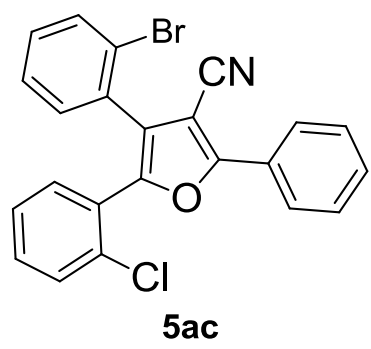

Prepared according to TP-A using $1 \mathbf{a}(93.6 \mathrm{mg}, 0.3 \mathrm{mmol}), 3(5.8 \mathrm{mg}, 0.03 \mathrm{mmol})$, $\mathrm{Et}_{3} \mathrm{~N}(8.4 \mu \mathrm{L}, 0.06 \mathrm{mmol}), \mathrm{PhSiH}_{3}(61 \mu \mathrm{L}, 0.48 \mathrm{mmol}), \mathbf{4 b}(10 \mu \mathrm{L}, 0.06 \mathrm{mmol}), \mathbf{2 c}$ $(42 \mu \mathrm{L}, 0.33 \mathrm{mmol})$ and THF $(1.5 \mathrm{~mL})$. The reaction mixture was stirred for 6 hours at $50{ }^{\circ} \mathrm{C}$. Then the solvent was removed by evaporation in vacuo, and crude mixture 
was purified by flash column chromatography $(\mathrm{DCM}$ :Hexanes $=1: 4)$ to provide 5ac as a white solid in $61 \%$ yield $(79.3 \mathrm{mg})$

mp.: 148.6-148.9 ${ }^{\circ} \mathrm{C}, \mathrm{R}_{f}=0.190 .(\mathrm{DCM}:$ Hexanes $=1: 4)$

${ }^{1} \mathbf{H}$ NMR $\left(400 \mathrm{MHz}, \mathrm{CDCl}_{3}, 25{ }^{\circ} \mathrm{C}\right) \delta / \mathrm{ppm}: 8.01(\mathrm{~d}, 2 \mathrm{H}, J=7.0 \mathrm{~Hz}), 7.66(\mathrm{~d}, 1 \mathrm{H}, J=$ $8.8 \mathrm{~Hz}), 7.41(\mathrm{t}, 2 \mathrm{H}, J=7.3 \mathrm{~Hz}), 7.38-7.32(\mathrm{~m}, 2 \mathrm{H}), 7.30-7.23(\mathrm{~m}, 4 \mathrm{H}), 7.16(\mathrm{~d}, 2 \mathrm{H}, J$ $=8.6 \mathrm{~Hz})$.

${ }^{13} \mathrm{C}$ NMR (100 MHz, $\left.\mathrm{CDCl}_{3}, 25{ }^{\circ} \mathrm{C}\right) \delta / \mathrm{ppm}: 158.2,148.0,134.7,133.7,131.8,131.4$, $130.9,130.5,129.2,129.1,128.3,127.8,127.5,126.6,125.5,124.5,123.6,114.0$, 96.4.

IR $(\mathrm{KBr}) \tilde{v}\left(\mathrm{~cm}^{-1}\right): 2228,1491,757,688$.

HRMS (EI) for $\mathbf{C}_{\mathbf{2 3}} \mathbf{H}_{\mathbf{1 3}} \mathbf{B r C I N O}[\mathbf{M}]^{+}$(432.9869) found 432.9869 .

\section{Synthesis of}

4-(2-bromophenyl)-5-(4-methoxyphenyl)-2-phenylfuran-3-carbonitrile (5ad)

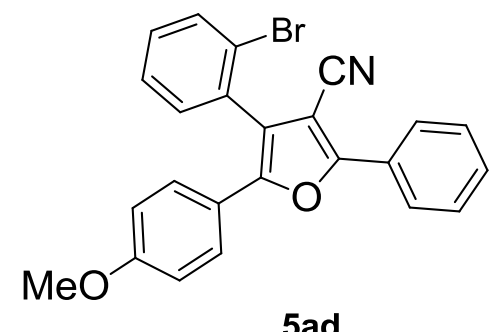

Prepared according to TP-A using 1a $(93.6 \mathrm{mg}, 0.3 \mathrm{mmol}), 3(5.8 \mathrm{mg}, 0.03 \mathrm{mmol})$, $\mathrm{Et}_{3} \mathrm{~N}(8.4 \mu \mathrm{L}, 0.06 \mathrm{mmol}), \mathrm{PhSiH}_{3}(61 \mu \mathrm{L}, 0.48 \mathrm{mmol}), \mathbf{4 b}(10 \mu \mathrm{L}, 0.06 \mathrm{mmol}), \mathbf{2 d}$ $(46 \mu \mathrm{L}, 0.33 \mathrm{mmol})$ and THF $(1.5 \mathrm{~mL})$. The reaction mixture was stirred for 12 hours at $50{ }^{\circ} \mathrm{C}$. Then the solvent was removed by evaporation in vacuo, and crude mixture was purified by flash column chromatography (DCM:Hexanes = 1:4) to provide 5ad as a white solid in $70 \%$ yield $(89.0 \mathrm{mg})$

mp.: 183.6-185.2 ${ }^{\circ} \mathrm{C}, \mathrm{R}_{f}=0.125 .(\mathrm{DCM}:$ Hexanes $=1: 4)$

${ }^{1} \mathbf{H}$ NMR $\left(400 \mathrm{MHz}, \mathrm{CDCl}_{3}, 25{ }^{\circ} \mathrm{C}\right) \delta / \mathrm{ppm}: 8.10(\mathrm{~d}, 2 \mathrm{H}, J=7.1 \mathrm{~Hz}), 7.76(\mathrm{~d}, 1 \mathrm{H}, J=$ 8.0 Hz), $7.51(\mathrm{t}, 2 \mathrm{H}, J=7.3 \mathrm{~Hz}), 7.48-7.39(\mathrm{~m}, 3 \mathrm{H}), 7.39-7.31(\mathrm{~m}, 3 \mathrm{H}), 6.83(\mathrm{~d}, 2 \mathrm{H}, J$ 
$=9.3 \mathrm{~Hz}), 3.79(\mathrm{~s}, 3 \mathrm{H})$.

${ }^{13} \mathrm{C}$ NMR (100 MHz, $\left.\mathrm{CDCl}_{3}, 25{ }^{\circ} \mathrm{C}\right) \delta / \mathrm{ppm}: 160.0,157.4,149.3,133.5,132.1,131.9$, 130.6, 130.1, 129.1, 128.1, 128.0, 127.0, 125.4, 124.8, 121.8, 121.5, 114.2, 96.1, 55.3.

IR $(\mathrm{KBr}) \tilde{v}\left(\mathrm{~cm}^{-1}\right): 2931,2227,1511,1255,767,688$.

HRMS (EI) for $\mathbf{C}_{\mathbf{2 4}} \mathbf{H}_{\mathbf{1 6}} \mathbf{B r N O}$ [M] $^{+}$(429.0364) found 429.0362 .

\section{Synthesis of 4-(2-bromophenyl)-2-phenyl-5-(p-tolyl)furan-3-carbonitrile (5ae)}

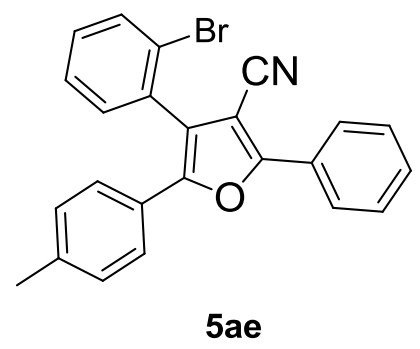

Prepared according to TP-A using $1 \mathbf{a}(93.6 \mathrm{mg}, 0.3 \mathrm{mmol}), 3(5.8 \mathrm{mg}, 0.03 \mathrm{mmol})$, $\mathrm{Et}_{3} \mathrm{~N}(8.4 \mu \mathrm{L}, 0.06 \mathrm{mmol}), \mathrm{PhSiH}_{3}(61 \mu \mathrm{L}, 0.48 \mathrm{mmol}), \mathbf{4 b}(10 \mu \mathrm{L}, 0.06 \mathrm{mmol}), \mathbf{2 e}$ $(45 \mu \mathrm{L}, 0.33 \mathrm{mmol})$ and THF $(1.5 \mathrm{~mL})$. The reaction mixture was stirred for 12 hours at $50{ }^{\circ} \mathrm{C}$. Then the solvent was removed by evaporation in vacuo, and crude mixture was purified by flash column chromatography (DCM:Hexanes = 1:5) to provide 5ae as a white solid in $77 \%$ yield $(95.6 \mathrm{mg})$

mp.: 183.6-185.2 ${ }^{\circ} \mathrm{C}, \mathrm{R}_{f}=0.250(\mathrm{DCM}:$ Hexanes $=1: 5)$

${ }^{1} \mathbf{H}$ NMR $\left(400 \mathrm{MHz}, \mathrm{CDCl}_{3}, 25{ }^{\circ} \mathrm{C}\right) \delta / \mathrm{ppm}: 8.13(\mathrm{~d}, 2 \mathrm{H}, J=7.5 \mathrm{~Hz}), 7.77(\mathrm{~d}, 1 \mathrm{H}, J=$ 8.0 Hz), $7.53(\mathrm{t}, 2 \mathrm{H}, J=7.5 \mathrm{~Hz}), 7.50-7.39(\mathrm{~m}, 3 \mathrm{H}), 7.39-7.28(\mathrm{~m}, 3 \mathrm{H}), 7.12(\mathrm{~d}, 2 \mathrm{H}, J$ $=8.2 \mathrm{~Hz}), 2.34(\mathrm{~s}, 3 \mathrm{H})$.

${ }^{13} \mathrm{C}$ NMR (100 MHz, $\left.\mathrm{CDCl}_{3}, 25{ }^{\circ} \mathrm{C}\right) \delta / \mathrm{ppm}: 157.6,149.3,138.9,133.4,131.9,131.8$, $130.6,130.1,129.4,129.1,128.0,127.9,126.2,125.4,125.3,125.2,124.6,122.3$, 114.2, 96.1, 21.3.

IR $(\mathrm{KBr}) \tilde{v}\left(\mathrm{~cm}^{-1}\right): 2227,1491,819,767,687$.

HRMS (EI) for $\mathbf{C}_{\mathbf{2 4}} \mathbf{H}_{\mathbf{1 6}} \mathbf{B r N O}[\mathbf{M}]^{+}$(413.0415) found 413.0417. 


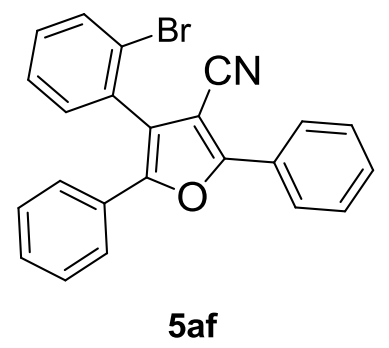

Prepared according to TP-A using 1a (93.6 mg, $0.3 \mathrm{mmol}), 3$ (5.8 mg, $0.03 \mathrm{mmol})$, $\mathrm{Et}_{3} \mathrm{~N}(8.4 \mu \mathrm{L}, 0.06 \mathrm{mmol}), \mathrm{PhSiH}_{3}(61 \mu \mathrm{L}, 0.48 \mathrm{mmol}), \mathbf{4 b}(10 \mu \mathrm{L}, 0.06 \mathrm{mmol}), \mathbf{2 f}(38$ $\mu \mathrm{L}, 0.33 \mathrm{mmol})$ and THF $(1.5 \mathrm{~mL})$. The reaction mixture was stirred for 12 hours at $50{ }^{\circ} \mathrm{C}$. Then the solvent was removed by evaporation in vacuo, and crude mixture was purified by flash column chromatography (DCM:Hexanes $=1: 5)$ to provide 5af as a white solid in $80 \%$ yield $(95.7 \mathrm{mg})$

mp.: 127.3-128.5 ${ }^{\circ} \mathrm{C}, \mathrm{R}_{f}=0.225 .(\mathrm{DCM}:$ Hexanes $=1: 5)$

${ }^{1} \mathbf{H}$ NMR $\left(400 \mathrm{MHz}, \mathrm{CDCl}_{3}, 25{ }^{\circ} \mathrm{C}\right) \delta / \mathrm{ppm}: 8.16(\mathrm{~d}, 2 \mathrm{H}, J=7.5 \mathrm{~Hz}), 7.80(\mathrm{~d}, 1 \mathrm{H}, J=$ $7.9 \mathrm{~Hz}), 7.56(\mathrm{t}, 2 \mathrm{H}, J=7.5 \mathrm{~Hz}), 7.53-7.36(\mathrm{~m}, 6 \mathrm{H}), 7.36-7.30(\mathrm{~m}, 3 \mathrm{H})$.

${ }^{13} \mathrm{C}$ NMR (100 MHz, $\left.\mathrm{CDCl}_{3}, 25{ }^{\circ} \mathrm{C}\right) \delta / \mathrm{ppm}: 157.7,148.8,133.4,131.8,131.5,130.6$, $130.1,129.0,128.8,128.6,128.0,127.7,125.3,125.2,124.4,122.9,114.0,96.1$.

IR $(\mathrm{KBr}) \tilde{v}\left(\mathrm{~cm}^{-1}\right): 2364,2227,1557,1491,767,687$.

HRMS (EI) for $\mathbf{C}_{\mathbf{2 3}} \mathbf{H}_{\mathbf{1 4}} \mathbf{B r N O}[\mathbf{M}]^{+}$(399.0259) found 399.0258.

Synthesis of 4-(2-bromophenyl)-5-cyclohexyl-2-phenylfuran-3-carbonitrile (5ag)

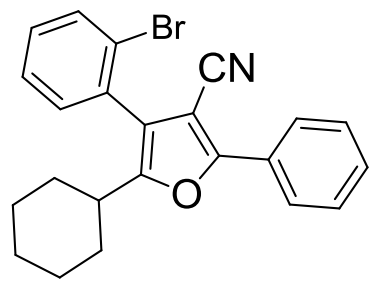

5 ag

Prepared according to TP-A using 1a $(93.6 \mathrm{mg}, 0.3 \mathrm{mmol}), 3(5.8 \mathrm{mg}, 0.03 \mathrm{mmol})$, $\mathrm{Et}_{3} \mathrm{~N}(8.4 \mu \mathrm{L}, 0.06 \mathrm{mmol}), \mathrm{PhSiH}_{3}(61 \mu \mathrm{L}, 0.48 \mathrm{mmol}), \mathbf{4 b}(10 \mu \mathrm{L}, 0.06 \mathrm{mmol}), \mathbf{2 g}$ $(46 \mu \mathrm{L}, 0.33 \mathrm{mmol})$ and THF $(1.5 \mathrm{~mL})$. The reaction mixture was stirred for 20 hours S10 
at $50{ }^{\circ} \mathrm{C}$. Then the solvent was removed by evaporation in vacuo, and crude mixture was purified by flash column chromatography (DCM:Hexanes = 1:3) to provide 5ag as a white solid in $33 \%$ yield $(39.8 \mathrm{mg})$

mp.: 107.0-108.2 ${ }^{\circ} \mathrm{C}, \mathrm{R}_{f}=0.250(\mathrm{DCM}:$ Hexanes $=1: 3)$

${ }^{1} \mathbf{H}$ NMR $\left(400 \mathrm{MHz}, \mathrm{CDCl}_{3}, 25{ }^{\circ} \mathrm{C}\right) \delta / \mathrm{ppm}: 8.02(\mathrm{~d}, 2 \mathrm{H}, J=8.4 \mathrm{~Hz}), 7.70(\mathrm{~d}, 1 \mathrm{H}, J=$ $8.0 \mathrm{~Hz}), 7.48(\mathrm{t}, 2 \mathrm{H}, J=7.5 \mathrm{~Hz}), 7.43(\mathrm{~d}, 1 \mathrm{H}, J=7.5 \mathrm{~Hz}), 7.39(\mathrm{~d}, 1 \mathrm{H}, J=7.0 \mathrm{~Hz})$, 7.34-7.24 (m, 2H), $2.56(\mathrm{tt}, 1 \mathrm{H}, J=11.9,3.2 \mathrm{~Hz}), 1.98-1.90(\mathrm{~m}, 1 \mathrm{H}), 1.84-1.73(\mathrm{~m}$, $3 \mathrm{H}), 1.73-1.53(\mathrm{~m}, 3 \mathrm{H}), 1.34-1.19(\mathrm{~m}, 3 \mathrm{H})$.

${ }^{13} \mathrm{C}$ NMR (100 MHz, $\left.\mathrm{CDCl}_{3}, 25{ }^{\circ} \mathrm{C}\right) \delta / \mathrm{ppm}: 157.4,156.8,133.2,132.1,131.5,130.2$, $129.8,129.0,128.4,127.5,125.2,124.8,121.5,114.7,94.3,36.6,31.6,30.8,26.1$, $26.0,25.7$.

IR $(\mathrm{KBr}) \tilde{v}\left(\mathrm{~cm}^{-1}\right): 2931,2855,2226,1561,767,690$.

HRMS (EI) for $\mathbf{C}_{23} \mathbf{H}_{20} \mathbf{B r N O}[\mathbf{M}]^{+}$(405.0728) found 405.0730

\section{Synthesis of 4-(2-bromophenyl)-2-phenyl-5-propylfuran-3-carbonitrile (5ah)}

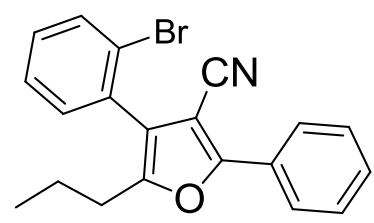

$5 \mathrm{ah}$

Prepared according to TP-A using $\mathbf{1 a}(93.6 \mathrm{mg}, 0.3 \mathrm{mmol}), 3(5.8 \mathrm{mg}, 0.03 \mathrm{mmol})$, $\mathrm{Et}_{3} \mathrm{~N}(8.4 \mu \mathrm{L}, 0.06 \mathrm{mmol}), \mathrm{PhSiH}_{3}(61 \mu \mathrm{L}, 0.48 \mathrm{mmol}), \mathbf{4 b}(10 \mu \mathrm{L}, 0.06 \mathrm{mmol}), \mathbf{2 h}$ $(35 \mu \mathrm{L}, 0.33 \mathrm{mmol})$ and THF $(1.5 \mathrm{~mL})$. The reaction mixture was stirred for 14 hours at $50{ }^{\circ} \mathrm{C}$. Then the solvent was removed by evaporation in vacuo, and crude mixture was purified by flash column chromatography (DCM:Hexanes $=1: 4)$ to provide 5ah as a white liquid in $66 \%$ yield $(70.9 \mathrm{mg})$.

mp.: 107.0-108.2 ${ }^{\circ} \mathrm{C}, \mathrm{R}_{f}=0.250 .(\mathrm{DCM}:$ Hexanes $=1: 4)$ 
${ }^{\mathbf{1}} \mathbf{H}$ NMR $\left(400 \mathrm{MHz}, \mathrm{CDCl}_{3}, 25{ }^{\circ} \mathrm{C}\right) \delta / \mathrm{ppm}: 8.02(\mathrm{~d}, 2 \mathrm{H}, J=8.8 \mathrm{~Hz}), 7.71(\mathrm{~d}, 1 \mathrm{H}, J=$ $8.0 \mathrm{~Hz}), 7.48(\mathrm{t}, 2 \mathrm{H}, J=7.5 \mathrm{~Hz}), 7.42(\mathrm{~d}, 1 \mathrm{H}, J=7.1 \mathrm{~Hz}), 7.39(\mathrm{~d}, 1 \mathrm{H}, J=7.1 \mathrm{~Hz})$, 7.36-7.24 (m, 2H), 2.69-2.49 (m, 2H), 1.77-1.62 (m, 2H), $0.93(\mathrm{t}, 3 \mathrm{H}, J=7.5 \mathrm{~Hz})$.

${ }^{13} \mathrm{C}$ NMR (100 MHz, $\left.\mathrm{CDCl}_{3}, 25{ }^{\circ} \mathrm{C}\right) \delta / \mathrm{ppm}: 157.8,153.2,133.2,132.1,131.2,130.2$, $129.8,129.0,128.3,127.6,125.2,124.7,123.0,114.7,94.2,28.6,21.2,13.7$.

IR $(\mathrm{KBr}) \tilde{v}\left(\mathrm{~cm}^{-1}\right):$ 2936, 2226, 1491, 767, 689.

HRMS (EI) for $\mathbf{C}_{\mathbf{2 0}} \mathbf{H}_{16} \mathbf{B r N O}[\mathbf{M}]^{+}$(365.0415) found 365.0416.

Synthesis of 4-(4-bromophenyl)-5-(4-chlorophenyl)-2-phenylfuran-3-carbonitrile (5ba)

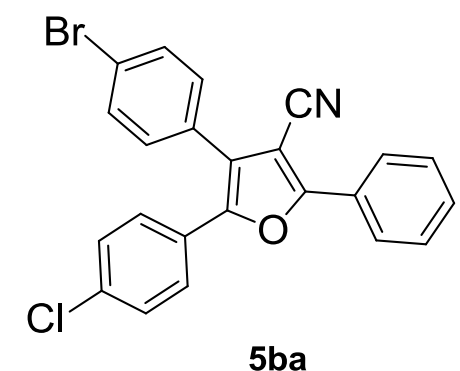

Prepared according to TP-A using $\mathbf{1 b}(93.6 \mathrm{mg}, 0.3 \mathrm{mmol}), \mathbf{3}(5.8 \mathrm{mg}, 0.03 \mathrm{mmol})$, $\mathrm{Et}_{3} \mathrm{~N}(8.4 \mu \mathrm{L}, 0.06 \mathrm{mmol}), \mathrm{PhSiH}_{3}(61 \mu \mathrm{L}, 0.48 \mathrm{mmol}), 4 \mathbf{b}(10 \mu \mathrm{L}, 0.06 \mathrm{mmol}), \mathbf{2 a}$ $(42 \mu \mathrm{L}, 0.33 \mathrm{mmol})$ and THF $(1.5 \mathrm{~mL})$. The reaction mixture was stirred for 6 hours at $50{ }^{\circ} \mathrm{C}$. Then the solvent was removed by evaporation in vacuo, and crude mixture was purified by flash column chromatography (DCM:Hexanes = 1:5) to provide $\mathbf{5 b a}$ as a white solid in $85 \%$ yield $(111.0 \mathrm{mg})$.

mp.: 187.5-188. ${ }^{\circ} \mathrm{C}, \mathrm{R}_{f}=0.350 .(\mathrm{DCM}:$ Hexanes $=1: 5)$

${ }^{\mathbf{1}} \mathbf{H}$ NMR $\left(400 \mathrm{MHz}, \mathrm{CDCl}_{3}, 25{ }^{\circ} \mathrm{C}\right) \delta / \mathrm{ppm}: 8.08(\mathrm{~d}, 2 \mathrm{H}, J=6.6 \mathrm{~Hz}), 7.61(\mathrm{~d}, 2 \mathrm{H}, J=$ 8.4 Hz), 7.56-7.47 (m, 3H), $7.44(\mathrm{~d}, 2 \mathrm{H}, J=8.4 \mathrm{~Hz}), 7.37-7.28(\mathrm{~m}, 4 \mathrm{H})$.

${ }^{13} \mathrm{C}$ NMR (100 MHz, $\left.\mathrm{CDCl}_{3}, 25{ }^{\circ} \mathrm{C}\right) \delta / \mathrm{ppm}: 158.8,147.6,134.9,132.5,130.8,130.5$, $129.2,129.1,128.8,127.6,127.4,127.2,125.5,123.3,123.2,114.2,95.4$.

IR $(\mathrm{KBr}) \tilde{v}\left(\mathrm{~cm}^{-1}\right): 2360,1492,831,769,687$.

HRMS (EI) for $\mathbf{C}_{23} \mathbf{H}_{13} \mathbf{B r C I N O}[\mathbf{M}]^{+}$(432.9869) found 432.9870 . 
Synthesis of 4-(2-chlorophenyl)-5-(4-chlorophenyl)-2-phenylfuran-3-carbonitrile (5ca)<smiles>N#Cc1c(-c2ccccc2)oc(-c2ccc(Cl)cc2)c1-c1ccccc1Cl</smiles>

Prepared according to TP-A using 1c $(80.3 \mathrm{mg}, 0.3 \mathrm{mmol}), 3(5.8 \mathrm{mg}, 0.03 \mathrm{mmol})$, $\mathrm{Et}_{3} \mathrm{~N}(8.4 \mu \mathrm{L}, 0.06 \mathrm{mmol}), \mathrm{PhSiH}_{3}(61 \mu \mathrm{L}, 0.48 \mathrm{mmol}), \mathbf{4 b}(10 \mu \mathrm{L}, 0.06 \mathrm{mmol}), \mathbf{2 a}$ $(42 \mu \mathrm{L}, 0.33 \mathrm{mmol})$ and THF $(1.5 \mathrm{~mL})$. The reaction mixture was stirred for 6 hours at $50{ }^{\circ} \mathrm{C}$. Then the solvent was removed by evaporation in vacuo, and crude mixture was purified by flash column chromatography (DCM:Hexanes $=1: 5)$ to provide $\mathbf{5 c a}$ as a white solid in $94 \%$ yield (109.7 mg).

mp.: 169.3-169.4 ${ }^{\circ} \mathrm{C}, \mathrm{R}_{f}=0.175 .(\mathrm{DCM}:$ Hexanes $=1: 5)$

${ }^{1} \mathbf{H}$ NMR $\left(400 \mathrm{MHz}, \mathrm{CDCl}_{3}, 25{ }^{\circ} \mathrm{C}\right) \delta / \mathrm{ppm}: 8.12(\mathrm{~d}, 2 \mathrm{H}, J=7.1 \mathrm{~Hz}), 7.59(\mathrm{~d}, 1 \mathrm{H}, J=$ $8.0 \mathrm{~Hz}), 7.54(\mathrm{~d}, 2 \mathrm{H}, J=7.3 \mathrm{~Hz}), 7.51-7.42(\mathrm{~m}, 2 \mathrm{H}), 7.42-7.39(\mathrm{~m}, 2 \mathrm{H}), 7.37(\mathrm{~d}, 2 \mathrm{H}, J$ $=8.8 \mathrm{~Hz}), 7.28(\mathrm{~d}, 2 \mathrm{H}, J=8.8 \mathrm{~Hz})$.

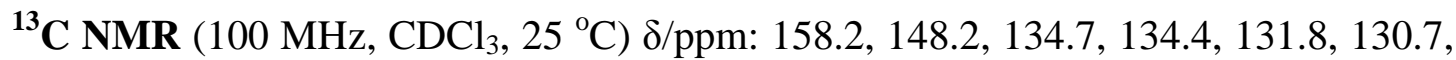
$130.4,129.2,129.1,129.0,127.7,127.6,127.5,126.5,125.5,121.7,113.9,96.4$.

IR $(\mathrm{KBr}) \tilde{v}\left(\mathrm{~cm}^{-1}\right): 2229,1492,831,755,687$.

HRMS (EI) for $\mathbf{C}_{23} \mathbf{H}_{13} \mathbf{C l}_{2} \mathbf{N O}$ [M] ${ }^{+}$(389.0374) found 389.0375.

Synthesis of 5-(4-chlorophenyl)-4-(4-nitrophenyl)-2-phenylfuran-3-carbonitrile $(\mathbf{5 d a})^{[\mathrm{a}]}$<smiles>N#Cc1c(-c2ccccc2)oc(-c2ccc(Cl)cc2)c1-c1ccc([N+](=O)[O-])cc1</smiles> 
Prepared according to TP-A using $\mathbf{1 d}(83.4 \mathrm{mg}, 0.3 \mathrm{mmol}), 3$ (5.8 mg, $0.03 \mathrm{mmol})$, $\mathrm{Et}_{3} \mathrm{~N}(8.4 \mu \mathrm{L}, 0.06 \mathrm{mmol}), \mathrm{PhSiH}_{3}(61 \mu \mathrm{L}, 0.48 \mathrm{mmol}), \mathbf{4 b}(10 \mu \mathrm{L}, 0.06 \mathrm{mmol}), \mathbf{2 a}$ $(42 \mu \mathrm{L}, 0.33 \mathrm{mmol})$ and THF $(1.5 \mathrm{~mL})$. The reaction mixture was stirred for 4 hours at $50{ }^{\circ} \mathrm{C}$. Then the solvent was removed by evaporation in vacuo, and crude mixture was purified by flash column chromatography (DCM:Hexanes $=1: 3)$ to provide 5da as a white solid in $98 \%$ yield $(117.9 \mathrm{mg})$.

mp.: 241.1-241.6 ${ }^{\circ} \mathrm{C}, \mathrm{R}_{f}=0.146 .(\mathrm{DCM}:$ Hexanes $=1: 3)$

${ }^{1} \mathbf{H}$ NMR $\left(400 \mathrm{MHz}, \mathrm{CDCl}_{3}, 25{ }^{\circ} \mathrm{C}\right) \delta / \mathrm{ppm}: 8.25(\mathrm{~d}, 2 \mathrm{H}, J=8.8 \mathrm{~Hz}), 8.01(\mathrm{~d}, 2 \mathrm{H}, J=$ $7.5 \mathrm{~Hz}), 7.59(\mathrm{~d}, 2 \mathrm{H}, J=8.8 \mathrm{~Hz}), 7.50-7.40(\mathrm{~m}, 3 \mathrm{H}), 7.35(\mathrm{~d}, 2 \mathrm{H}, J=8.8 \mathrm{~Hz}), 7.26(\mathrm{~d}$, $2 \mathrm{H}, J=8.4 \mathrm{~Hz})$.

${ }^{13} \mathrm{C}$ NMR (100 MHz, $\left.\mathrm{CDCl}_{3}, 25{ }^{\circ} \mathrm{C}\right) \delta / \mathrm{ppm}: 159.4,148.6,147.9,136.7,135.6,130.8$, $130.2,129.3,129.2,127.8,127.3,126.7,125.6,124.5,122.1,113.9,94.9$.

IR $(\mathrm{KBr}) \tilde{v}\left(\mathrm{~cm}^{-1}\right): 2227,1519,1492,1345,769,688$.

HRMS (EI) for $\mathbf{C}_{23} \mathbf{H}_{13} \mathbf{C I N}_{2} \mathbf{O}_{3}[\mathbf{M}]^{+}$(400.0615) found 400.0616 .

\section{Synthesis of 5-(4-chlorophenyl)-2,4-diphenylfuran-3-carbonitrile (5ea)}

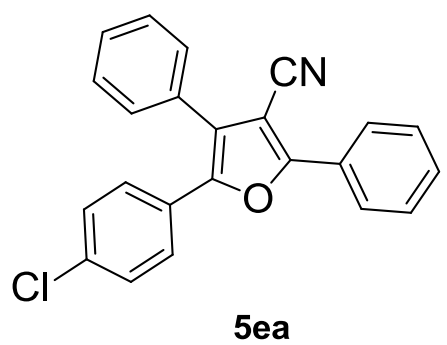

Prepared according to TP-A using 1e (70.0 mg, $0.3 \mathrm{mmol}), 3(5.8 \mathrm{mg}, 0.03 \mathrm{mmol})$, $\mathrm{Et}_{3} \mathrm{~N}(8.4 \mu \mathrm{L}, 0.06 \mathrm{mmol}), \mathrm{PhSiH}_{3}(61 \mu \mathrm{L}, 0.48 \mathrm{mmol}), \mathbf{4 b}(10 \mu \mathrm{L}, 0.06 \mathrm{mmol}), \mathbf{2 a}$ $(42 \mu \mathrm{L}, 0.33 \mathrm{mmol})$ and $\mathrm{THF}(1.5 \mathrm{~mL})$. The reaction mixture was stirred for 12 hours at $50{ }^{\circ} \mathrm{C}$. Then the solvent was removed by evaporation in vacuo, and crude mixture was purified by flash column chromatography (DCM:Hexanes $=1: 5)$ to provide 5ea as a white solid in $93 \%$ yield (100.1 mg). 
mp.: 161.3-161. ${ }^{\circ} \mathrm{C}, \mathrm{R}_{f}=0.250(\mathrm{DCM}:$ Hexanes $=1: 5)$

${ }^{1} \mathbf{H}$ NMR $\left(400 \mathrm{MHz}, \mathrm{CDCl}_{3}, 25{ }^{\circ} \mathrm{C}\right) \delta / \mathrm{ppm}: 8.09(\mathrm{~d}, 2 \mathrm{H}, J=8.4 \mathrm{~Hz}), 7.51(\mathrm{~d}, 2 \mathrm{H}, J=$ $7.1 \mathrm{~Hz}), 7.48-7.41(\mathrm{~m}, 8 \mathrm{H}), 7.27(\mathrm{~d}, 2 \mathrm{H}, J=8.8 \mathrm{~Hz})$.

${ }^{13} \mathrm{C}$ NMR (100 MHz, $\left.\mathrm{CDCl}_{3}, 25{ }^{\circ} \mathrm{C}\right) \delta / \mathrm{ppm}: 158.5,147.4,134.5,130.3,129.9,129.2$, $129.1,128.9,127.8,127.5,127.3,125.5,124.5,114.4,95.7$.

IR $(\mathrm{KBr}) \tilde{v}\left(\mathrm{~cm}^{-1}\right): 2362,2226,1479,831,768,688$.

HRMS (EI) for $\mathbf{C}_{\mathbf{2 3}} \mathbf{H}_{\mathbf{1 4}}$ CINO [M] ${ }^{+}$(355.0764) found 355.0764.

\section{Synthesis of 5-(4-chlorophenyl)-2-phenyl-4-(thiophen-2-yl)furan-3-carbonitrile} $(\mathbf{5 f a})^{[\mathrm{a}]}$

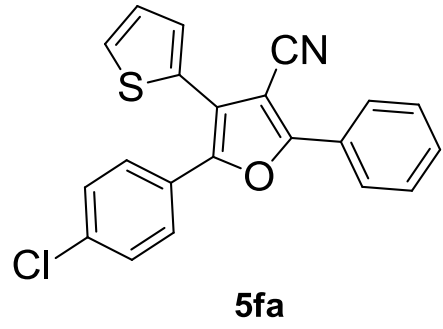

Prepared according to TP-A using $\mathbf{1 f}(71.8 \mathrm{mg}, 0.3 \mathrm{mmol}), 3(5.8 \mathrm{mg}, 0.03 \mathrm{mmol})$, $\mathrm{Et}_{3} \mathrm{~N}(8.4 \mu \mathrm{L}, 0.06 \mathrm{mmol}), \mathrm{PhSiH}_{3}(61 \mu \mathrm{L}, 0.48 \mathrm{mmol}), 4 \mathbf{b}(10 \mu \mathrm{L}, 0.06 \mathrm{mmol}), \mathbf{2 a}$ $(42 \mu \mathrm{L}, 0.33 \mathrm{mmol})$ and THF $(1.5 \mathrm{~mL})$. The reaction mixture was stirred for 12 hours at $50{ }^{\circ} \mathrm{C}$. Then the solvent was removed by evaporation in vacuo, and crude mixture was purified by flash column chromatography (DCM:Hexanes $=1: 4)$ to provide $\mathbf{5 f a}$ as a white solid in $89 \%$ yield (96.1 mg).

mp.: 1676-168. ${ }^{\circ} \mathrm{C}, \mathrm{R}_{f}=0.350 .(\mathrm{DCM}:$ Hexanes $=1: 4)$

${ }^{1} \mathbf{H}$ NMR $\left(400 \mathrm{MHz}, \mathrm{CDCl}_{3}, 25{ }^{\circ} \mathrm{C}\right) \delta / \mathrm{ppm}: 8.10(\mathrm{~d}, 2 \mathrm{H}, J=7.5 \mathrm{~Hz}), 7.56-7.48(\mathrm{~m}$, $6 \mathrm{H}), 7.32(\mathrm{~d}, 2 \mathrm{H}, J=8.8 \mathrm{~Hz}), 7.29-7.24(\mathrm{~m}, 1 \mathrm{H}), 7.18-7.13(\mathrm{~m}, 1 \mathrm{H})$.

${ }^{13} \mathrm{C}$ NMR (100 MHz, $\left.\mathrm{CDCl}_{3}, 25{ }^{\circ} \mathrm{C}\right) \delta / \mathrm{ppm}: 158.6,148.5,135.1,130.5,129.8,129.2$, $129.0,128.6,127.9,127.8,127.6,127.5,127.1,125.5,117.8,114.2,96.2$.

IR $(\mathrm{KBr}) \tilde{v}\left(\mathrm{~cm}^{-1}\right):$ 2361, 2227,484, 1096, 830, 768, 686.

HRMS (EI) for $\mathbf{C}_{\mathbf{2 1}} \mathbf{H}_{\mathbf{1 2}} \mathbf{C I N O S}[\mathbf{M}]^{+}$(361.0328) found 361.0330 . 


\section{Synthesis of}

5-(4-chlorophenyl)-4-(4-methoxyphenyl)-2-phenylfuran-3-carbonitrile (5ga)

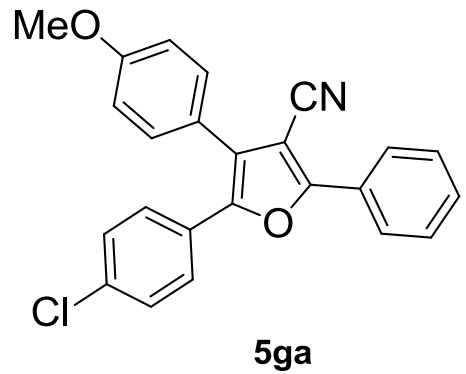

Prepared according to TP-A using $1 \mathbf{g}(78.9 \mathrm{mg}, 0.3 \mathrm{mmol}), 3(5.8 \mathrm{mg}, 0.03 \mathrm{mmol})$, $\mathrm{Et}_{3} \mathrm{~N}(8.4 \mu \mathrm{L}, 0.06 \mathrm{mmol}), \mathrm{PhSiH}_{3}(61 \mu \mathrm{L}, 0.48 \mathrm{mmol}), \mathbf{4 b}(10 \mu \mathrm{L}, 0.06 \mathrm{mmol}), \mathbf{2 a}$ $(42 \mu \mathrm{L}, 0.33 \mathrm{mmol})$ and THF $(1.5 \mathrm{~mL})$. The reaction mixture was stirred for 12 hours at $50{ }^{\circ} \mathrm{C}$. Then the solvent was removed by evaporation in vacuo, and crude mixture was purified by flash column chromatography (DCM:Hexanes = 1:5) to provide 5ga as a yellow solid in $63 \%$ yield $(73.0 \mathrm{mg})$.

mp.: 1953-196.5 ${ }^{\circ} \mathrm{C} ; \mathrm{R}_{f}=0.125$ (DCM:Hexanes $\left.=1: 5\right)$

${ }^{1} \mathbf{H}$ NMR $\left(400 \mathrm{MHz}, \mathrm{CDCl}_{3}, 25{ }^{\circ} \mathrm{C}\right) \delta / \mathrm{ppm}: 8.10(\mathrm{~d}, 2 \mathrm{H}, J=7.1 \mathrm{~Hz}), 7.54-7.44(\mathrm{~d}, 5 \mathrm{H}$, $J=8.4 \mathrm{~Hz}), 7.38(\mathrm{~d}, 2 \mathrm{H}, J=8.8 \mathrm{~Hz}), 7.28(\mathrm{~d}, 2 \mathrm{H}, J=8.8 \mathrm{~Hz}), 7.00(\mathrm{~d}, 2 \mathrm{H}, J=8.8$ $\mathrm{Hz}), 3.87$ (s, 3H).

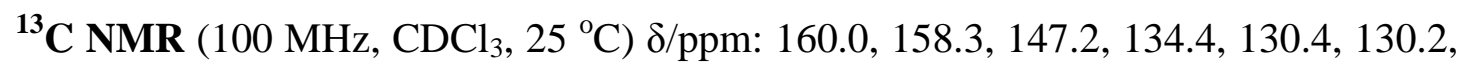
129.1, 128.9, 127.9, 127.7, 127.1, 125.4, 124.3, 121.9, 114.7, 114.6, 95.9, 55.3.

IR $(\mathrm{KBr}) \tilde{v}\left(\mathrm{~cm}^{-1}\right): 2227,1513,1250,832,770,687$.

HRMS (EI) for $\mathbf{C}_{\mathbf{2 4}} \mathbf{H}_{\mathbf{1 6}} \mathbf{C I N O}_{\mathbf{2}}[\mathbf{M}]^{+}$(385.0870) found 385.0870.

Synthesis of ethyl 5-(4-chlorophenyl)-4-(4-nitrophenyl)-2-phenylfuran-3carboxylate $(7 \mathbf{a a})^{[\mathrm{a}]}$<smiles>CCOC(=O)c1c(-c2ccccc2)oc(-c2ccc(Cl)cc2)c1-c1ccc([18O])cc1</smiles> 
Prepared according to TP-A using $6 \mathbf{a}(97.6 \mathrm{mg}, 0.3 \mathrm{mmol}), 3(5.8 \mathrm{mg}, 0.03 \mathrm{mmol})$, $\mathrm{Et}_{3} \mathrm{~N}(8.4 \mu \mathrm{L}, 0.06 \mathrm{mmol}), \mathrm{PhSiH}_{3}(61 \mu \mathrm{L}, 0.48 \mathrm{mmol}), 4 \mathbf{b}(10 \mu \mathrm{L}, 0.06 \mathrm{mmol}), \mathbf{2 a}$ (42 $\mu \mathrm{L}, 0.33 \mathrm{mmol})$ and THF $(1.5 \mathrm{~mL})$. The reaction mixture was stirred for 9 hours at $50{ }^{\circ} \mathrm{C}$. Then the solvent was removed by evaporation in vacuo, and crude mixture was purified by flash column chromatography (DCM:Hexs $=1: 5)$ to provide 7aa as a yellow solid in $85 \%$ yield $(79.6 \mathrm{mg})$.

mp.: $172.9-173.5^{\circ} \mathrm{C}, \mathrm{R}_{f}=0.100(\mathrm{DCM}: \mathrm{Hexs}=1: 5)$

${ }^{1} \mathbf{H}$ NMR (400 MHz, $\left.\mathrm{CDCl}_{3}, 25{ }^{\circ} \mathrm{C}\right) \delta / \mathrm{ppm}: 8.29(\mathrm{~d}, 2 \mathrm{H}, J=8.7 \mathrm{~Hz}), 7.90(\mathrm{~d}, 2 \mathrm{H}, J=$ $7.5 \mathrm{~Hz}), 7.56(\mathrm{~d}, 2 \mathrm{H}, J=8.7 \mathrm{~Hz}), 7.50-7.44(\mathrm{~m}, 3 \mathrm{H}), 7.30-7.24(\mathrm{~m}, 4 \mathrm{H}), 4.09(\mathrm{q}, 2 \mathrm{H}$, $J=7.2 \mathrm{~Hz}), 0.99(\mathrm{t}, 3 \mathrm{H}, J=7.2 \mathrm{~Hz})$.

${ }^{13} \mathrm{C}$ NMR (100 MHz, $\left.\mathrm{CDCl}_{3}, 25{ }^{\circ} \mathrm{C}\right) \delta / \mathrm{ppm}: 163.3,156.0,147.9,147.5,140.4,134.4$, 131.1, 129.7, 129.2, 128.9, 128.3, 128.2, 127.7, 127.2, 123.7, 121.9, 116.1, 60.8, 13.6.

IR (KBr) $\tilde{v}\left(\mathrm{~cm}^{-1}\right): 2980(\mathrm{w}), 1719(\mathrm{~s}), 1517(\mathrm{~s}), 1346(\mathrm{~s}), 1230(\mathrm{~m}), 1119(\mathrm{~m}), 855$ (m), $691(\mathrm{~m})$.

HRMS (EI) for $\mathbf{C}_{25} \mathbf{H}_{18} \mathbf{N O}_{\mathbf{5}} \mathbf{C l},[\mathbf{M}]^{+}$(447.0874) found: 447.0877.

Synthesis of ethyl 5-(3-chlorophenyl)-4-(4-nitrophenyl)-2-phenylfuran-3carboxylate $(7 \mathbf{a b})^{[\mathrm{a}]}$

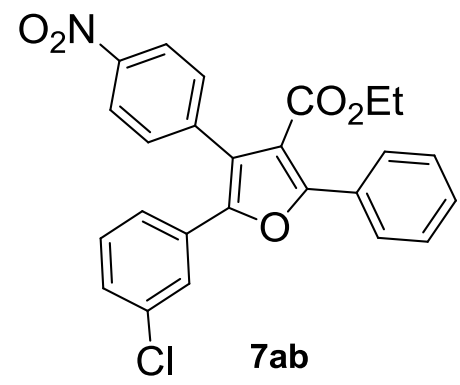

Prepared according to TP-A using 6a (97.6 mg, $0.3 \mathrm{mmol}), 3(5.8 \mathrm{mg}, 0.03 \mathrm{mmol})$, $\mathrm{Et}_{3} \mathrm{~N}(8.4 \mu \mathrm{L}, 0.06 \mathrm{mmol}), \mathrm{PhSiH}_{3}(61 \mu \mathrm{L}, 0.48 \mathrm{mmol}), \mathbf{4 b}(10 \mu \mathrm{L}, 0.06 \mathrm{mmol}), \mathbf{2 b}$ $(43 \mu \mathrm{L}, 0.33 \mathrm{mmol})$ and THF $(1.5 \mathrm{~mL})$. The reaction mixture was stirred for 7 hours 
at $50{ }^{\circ} \mathrm{C}$. Then the solvent was removed by evaporation in vacuo, and crude mixture was purified by flash column chromatography (DCM:Hexs $=1: 5)$ to provide 7ab as a yellow solid in $92 \%$ yield $(123.4 \mathrm{mg})$.

mp.: 133.6-134.1 ${ }^{\circ} \mathrm{C}, \mathrm{R}_{f}=0.100(\mathrm{DCM}: \mathrm{Hexs}=1: 5)$

${ }^{1} \mathbf{H}$ NMR $\left(400 \mathrm{MHz}, \mathrm{CDCl}_{3}, 25{ }^{\circ} \mathrm{C}\right) \delta / \mathrm{ppm}: 8.31(\mathrm{~d}, 2 \mathrm{H}, J=8.6 \mathrm{~Hz}), 7.92(\mathrm{~d}, 2 \mathrm{H}, J=$ $6.3 \mathrm{~Hz}), 7.57(\mathrm{~d}, 2 \mathrm{H}, J=8.6 \mathrm{~Hz}), 7.51-7.46(\mathrm{~m}, 4 \mathrm{H}), 7.26-7.23(\mathrm{~m}, 1 \mathrm{H}), 7.17(\mathrm{t}, 1 \mathrm{H}, J$ $=7.8 \mathrm{~Hz}), 7.10(\mathrm{~d}, 1 \mathrm{H}, J=7.8 \mathrm{~Hz}), 4.10(\mathrm{q}, 2 \mathrm{H}, J=7.1 \mathrm{~Hz}), 1.00(\mathrm{t}, 3 \mathrm{H}, J=7.1 \mathrm{~Hz})$. ${ }^{13} \mathrm{C}$ NMR (100 MHz, $\left.\mathrm{CDCl}_{3}, 25{ }^{\circ} \mathrm{C}\right) \delta / \mathrm{ppm}: 163.3,156.2,147.5,147.4,140.2,134.8$, $131.1,130.9,129.9,129.8,129.1,128.4,128.3,128.2,126.0,124.0,123.7,122.5$, $116.1,60.9,13.6$.

IR $(\mathrm{KBr}) \tilde{v}\left(\mathrm{~cm}^{-1}\right): 2984,1719,1520,1346,1232,1105,851,691$.

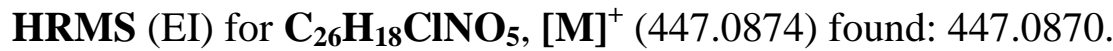

\section{Synthesis of ethyl 5-(2-chlorophenyl)-4-(4-nitrophenyl)-2-phenylfuran-3-} carboxylate $(7 \mathbf{a c})^{[\mathrm{a}]}$

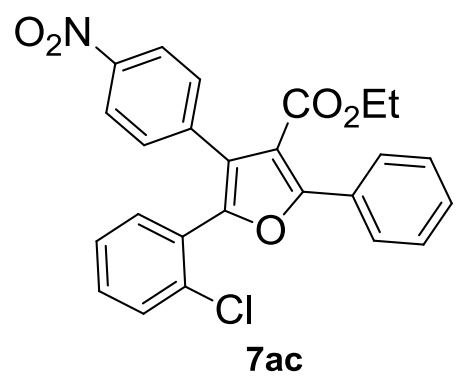

Prepared according to TP-A using 6a $(97.6 \mathrm{mg}, 0.3 \mathrm{mmol}), 3(5.8 \mathrm{mg}, 0.03 \mathrm{mmol})$, $\mathrm{Et}_{3} \mathrm{~N}(8.4 \mu \mathrm{L}, 0.06 \mathrm{mmol}), \mathrm{PhSiH}_{3}(61 \mu \mathrm{L}, 0.48 \mathrm{mmol}), \mathbf{4 b}(10 \mu \mathrm{L}, 0.06 \mathrm{mmol}), \mathbf{2 c}$ (42 $\mu \mathrm{L}, 0.33 \mathrm{mmol})$ and THF $(1.5 \mathrm{~mL})$. The reaction mixture was stirred for 9 hours at $50{ }^{\circ} \mathrm{C}$. Then the solvent was removed by evaporation in vacuo, and crude mixture was purified by flash column chromatography (DCM:Hexs $=1: 5)$ to provide 7ac as a yellow solid in $88 \%$ yield $(117.7 \mathrm{mg})$.

mp.: $110.4-110.9^{\circ} \mathrm{C}, \mathrm{R}_{f}=0.100(\mathrm{DCM}: \mathrm{Hexs}=1: 5)$

${ }^{1} \mathbf{H}$ NMR $\left(400 \mathrm{MHz}, \mathrm{CDCl}_{3}, 25{ }^{\circ} \mathrm{C}\right) \delta / \mathrm{ppm}: 8.15(\mathrm{~d}, 2 \mathrm{H}, J=8.8 \mathrm{~Hz}), 7.92(\mathrm{~d}, 2 \mathrm{H}, J=$ $\mathrm{S} 18$ 
$8.0 \mathrm{~Hz}), 7.47-7.42(\mathrm{~m}, 6 \mathrm{H}), 7.32(\mathrm{t}, 1 \mathrm{H}, J=8.6 \mathrm{~Hz}), 7.26-7.20(\mathrm{~m}, 2 \mathrm{H}), 4.18(\mathrm{q}, 2 \mathrm{H}, J$ $=7.0 \mathrm{~Hz}), 1.07(\mathrm{t}, 3 \mathrm{H}, J=7.0 \mathrm{~Hz})$.

${ }^{13} \mathrm{C}$ NMR (100 MHz, $\left.\mathrm{CDCl}_{3}, 25{ }^{\circ} \mathrm{C}\right) \delta / \mathrm{ppm}: 163.7,156.7,147.9,147.0,139.6,134.1$, $132.2,130.8,130.6,130.4,129.6,129.3,128.4,128.3,128.2,126.8,124.2,123.2$, $114.5,61.0,13.7$.

IR $(\mathrm{KBr}) \tilde{v}\left(\mathrm{~cm}^{-1}\right): 2984,1721,1520,1346,1230,1099,855,730$.

HRMS (MALDI) for $\mathbf{C}_{26} \mathbf{H}_{19} \mathbf{C I N O}_{5}$, [M+H] $^{+}$(448.0952) found: 448.0965 .

\section{Synthesis of ethyl 5-(4-methoxyphenyl)-4-(4-nitrophenyl)-2-phenylfuran-3-} carboxylate $(7 \mathbf{a d})^{[\mathrm{a}]}$

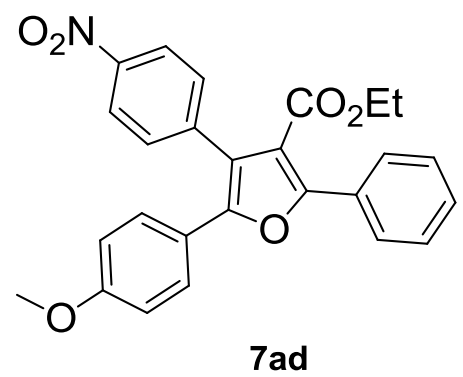

Prepared according to TP-A using $\mathbf{6 a}(97.6 \mathrm{mg}, 0.3 \mathrm{mmol}), 3(5.8 \mathrm{mg}, 0.03 \mathrm{mmol})$, $\mathrm{Et}_{3} \mathrm{~N}(8.4 \mu \mathrm{L}, 0.06 \mathrm{mmol}), \mathrm{PhSiH}_{3}(61 \mu \mathrm{L}, 0.48 \mathrm{mmol}), \mathbf{4 b}(10 \mu \mathrm{L}, 0.06 \mathrm{mmol}), \mathbf{2 d}$ $(46 \mu \mathrm{L}, 0.33 \mathrm{mmol})$ and $\mathrm{THF}(1.5 \mathrm{~mL})$. The reaction mixture was stirred for 18 hours at $50{ }^{\circ} \mathrm{C}$. Then the solvent was removed by evaporation in vacuo, and crude mixture was purified by flash column chromatography (DCM:Hexs $=1: 1)$ to provide 7ad as a yellow solid in $62 \%$ yield $(81.9 \mathrm{mg})$.

mp.: 151.1-151.6 ${ }^{\circ} \mathrm{C}, \mathrm{R}_{f}=0.190(\mathrm{DCM}: \mathrm{Hexs}=1: 1)$

${ }^{1} \mathbf{H}$ NMR $\left(400 \mathrm{MHz}, \mathrm{CDCl}_{3}, 25{ }^{\circ} \mathrm{C}\right) \delta / \mathrm{ppm}: 8.27(\mathrm{~d}, 2 \mathrm{H}, J=8.6 \mathrm{~Hz}), 7.92(\mathrm{~d}, 2 \mathrm{H}, J=$ $7.2 \mathrm{~Hz}), 7.55(\mathrm{~d}, 2 \mathrm{H}, J=8.6 \mathrm{~Hz}), 7.49-7.43(\mathrm{~m}, 3 \mathrm{H}), 7.30(\mathrm{~d}, 2 \mathrm{H}, J=8.7 \mathrm{~Hz}), 6.81(\mathrm{~d}$, $2 \mathrm{H}, J=8.7 \mathrm{~Hz}), 4.09(\mathrm{q}, 2 \mathrm{H}, J=7.1 \mathrm{~Hz}), 3.78(\mathrm{~s}, 3 \mathrm{H}), 1.00(\mathrm{t}, 3 \mathrm{H}, J=7.1 \mathrm{~Hz})$.

${ }^{13} \mathrm{C}$ NMR $\left(100 \mathrm{MHz}, \mathrm{CDCl} 3,25{ }^{\circ} \mathrm{C}\right) \delta / \mathrm{ppm}: 163.5,159.7,155.1,149.2,147.1,140.9$, $131.2,129.5,129.3,128.2,128.0,127.6,123.5,121.8,119.9,115.8,114.1,60.6,55.2$ 
13.6.

IR (KBr) $\tilde{v}\left(\mathrm{~cm}^{-1}\right):$ 2980, 2962, 1715, 1515, 1349, 1254, 1180, 1112, 833, 693.

HRMS (EI) for $\mathbf{C}_{26} \mathbf{H}_{21} \mathbf{N O}_{6}$, [M] $]^{+}$(443.1369) found: 443.1373.

Synthesis of ethyl 4-(4-nitrophenyl)-2-phenyl-5-p-tolylfuran-3-carboxylate (7ae):

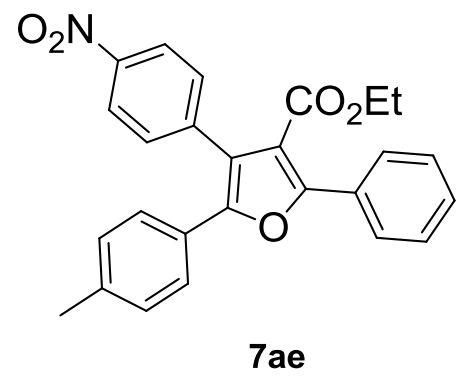

Prepared according to TP-A using 6a (97.6 mg, $0.3 \mathrm{mmol}), 3(5.8 \mathrm{mg}, 0.03 \mathrm{mmol})$, $\mathrm{Et}_{3} \mathrm{~N}(8.4 \mu \mathrm{L}, 0.06 \mathrm{mmol}), \mathrm{PhSiH}_{3}(61 \mu \mathrm{L}, 0.48 \mathrm{mmol}), \mathbf{4 b}(10 \mu \mathrm{L}, 0.06 \mathrm{mmol}), \mathbf{2 e}$ $(45 \mu \mathrm{L}, 0.33 \mathrm{mmol})$ and THF $(1.5 \mathrm{~mL})$. The reaction mixture was stirred for 15 hours at $50{ }^{\circ} \mathrm{C}$. Then the solvent was removed by evaporation in vacuo, and crude mixture was purified by flash column chromatography (DCM:Hexs $=1: 5)$ to provide 7ae as a yellow solid in $73 \%$ yield $(93.8 \mathrm{mg})$.

mp.: $145.4-146.2{ }^{\circ} \mathrm{C}, \mathrm{R}_{f}=0.100(\mathrm{DCM}: \mathrm{Hexs}=1: 5)$

${ }^{\mathbf{1}} \mathbf{H}$ NMR $\left(400 \mathrm{MHz}, \mathrm{CDCl}_{3}, 25{ }^{\circ} \mathrm{C}\right) \delta / \mathrm{ppm}: 8.28(\mathrm{~d}, 2 \mathrm{H}, J=8.7 \mathrm{~Hz}), 7.92(\mathrm{~d}, 2 \mathrm{H}, J=$ $8.2 \mathrm{~Hz}), 7.56(\mathrm{~d}, 2 \mathrm{H}, J=8.7 \mathrm{~Hz}), 7.50-7.44(\mathrm{~m}, 3 \mathrm{H}), 7.25(\mathrm{~d}, 2 \mathrm{H}, J=7.8 \mathrm{~Hz}), 7.09(\mathrm{~d}$, $2 \mathrm{H}, J=8.2 \mathrm{~Hz}), 4.09(\mathrm{q}, 2 \mathrm{H}, J=7.1 \mathrm{~Hz}), 2.33(\mathrm{~s}, 3 \mathrm{H}), 1.00(\mathrm{t}, 3 \mathrm{H}, J=7.1 \mathrm{~Hz})$.

${ }^{13} \mathrm{C}$ NMR (100 MHz, CDCl3, $\left.25{ }^{\circ} \mathrm{C}\right) \delta / \mathrm{ppm}: 163.6,155.4,149.4,147.3,140.9,138.7$, $131.3,129.6,129.5,129.4,128.3,128.2,126.5,126.2,123.6,120.8,115.9,60.7,21.3$, 13.7.

IR $(\mathrm{KBr}) \tilde{v}\left(\mathrm{~cm}^{-1}\right): 2980,1715,1517,1344,1230,1114,853,691$.

HRMS (EI) for $\mathbf{C}_{26} \mathbf{H}_{21} \mathbf{N O}_{5},[M]^{+}$(427.1420) found: 427.1428 . 


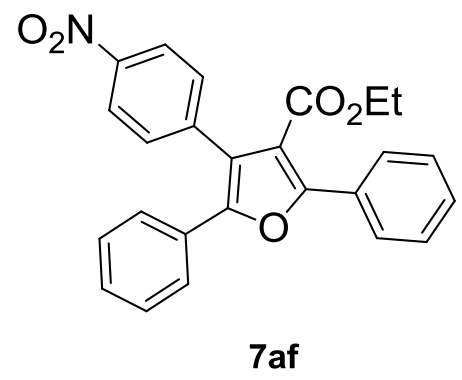

Prepared according to TP-A using $6 \mathbf{a}(97.6 \mathrm{mg}, 0.3 \mathrm{mmol}), 3(5.8 \mathrm{mg}, 0.03 \mathrm{mmol})$, $\mathrm{Et}_{3} \mathrm{~N}(8.4 \mu \mathrm{L}, 0.06 \mathrm{mmol}), \mathrm{PhSiH}_{3}(61 \mu \mathrm{L}, 0.48 \mathrm{mmol}), \mathbf{4 b}(10 \mu \mathrm{L}, 0.06 \mathrm{mmol}), 2 \mathbf{f}(38$ $\mu \mathrm{L}, 0.33 \mathrm{mmol})$ and THF $(1.5 \mathrm{~mL})$. The reaction mixture was stirred for 12 hours at $50{ }^{\circ} \mathrm{C}$. Then the solvent was removed by evaporation in vacuo, and crude mixture was purified by flash column chromatography (DCM:Hexs $=1: 5$ ) to provide 7af as a yellow solid in $82 \%$ yield $(101.6 \mathrm{mg})$.

mp.: $181.8-182.4{ }^{\circ} \mathrm{C}, \mathrm{R}_{f}=0.100(\mathrm{DCM}: \mathrm{Hexs}=1: 5)$

${ }^{1} \mathbf{H}$ NMR $\left(400 \mathrm{MHz}, \mathrm{CDCl}_{3}, 25{ }^{\circ} \mathrm{C}\right) \delta / \mathrm{ppm}: 8.28(\mathrm{~d}, 2 \mathrm{H}, J=8.6 \mathrm{~Hz}), 7.92(\mathrm{~d}, 2 \mathrm{H}, J=$ $7.8 \mathrm{~Hz}), 7.57(\mathrm{~d}, 2 \mathrm{H}, J=8.6 \mathrm{~Hz}), 7.49-7.48(\mathrm{~m}, 3 \mathrm{H}), 7.38-7.35(\mathrm{~m}, 2 \mathrm{H})$, 7.29-7.27 (m, $3 \mathrm{H}), 4.10(\mathrm{q}, 2 \mathrm{H}, J=7.1 \mathrm{~Hz}), 1.01(\mathrm{t}, 3 \mathrm{H}, J=7.1 \mathrm{~Hz})$.

${ }^{13} \mathrm{C}$ NMR $\left(100 \mathrm{MHz}, \mathrm{CDCl} 3,25{ }^{\circ} \mathrm{C}\right) \delta / \mathrm{ppm}: 163.5,155.7,149.1,147.4,140.8,131.2$, $129.6,129.4,129.3,128.7,128.5,128.3,128.2,126.2,123.6,121.5,116.0,60.8,13.7$.

IR $(\mathrm{KBr}) \tilde{v}\left(\mathrm{~cm}^{-1}\right): 2984,1717,1520,1346,1230,1116,855,691$.

HRMS (EI) for $\mathbf{C}_{25} \mathbf{H}_{19} \mathbf{N O}_{5}$, [M] $]^{+}$(413.1263) found: 413.1256 .

Synthesis of ethyl 4,5-bis(4-nitrophenyl)-2-phenylfuran-3-carboxylate $\left(\right.$ 7ai) ${ }^{[\mathrm{a}]}$<smiles>CCOC(=O)c1c(-c2ccccc2)oc(-c2ccc([N+](=O)[O-])cc2)c1-c1ccc([N+](=O)[O-])cc1</smiles> 
Prepared according to TP-A using $6 \mathbf{a}(97.6 \mathrm{mg}, 0.3 \mathrm{mmol}), 3(5.8 \mathrm{mg}, 0.03 \mathrm{mmol})$, $\mathrm{Et}_{3} \mathrm{~N}(8.4 \mu \mathrm{L}, 0.06 \mathrm{mmol}), \mathrm{PhSiH}_{3}(61 \mu \mathrm{L}, 0.48 \mathrm{mmol}), \mathbf{4 b}(10 \mu \mathrm{L}, 0.06 \mathrm{mmol}), 2 \mathbf{i}$ (63 $\mathrm{mg}, 0.33 \mathrm{mmol})$ and THF $(1.5 \mathrm{~mL})$. The reaction mixture was stirred for 9 hours at 50 ${ }^{\circ} \mathrm{C}$. Then the solvent was removed by evaporation in vacuo, and crude mixture was purified by flash column chromatography (DCM:Hexs $=1: 1)$ to provide 7ai as a yellow solid in $91 \%$ yield $(125.2 \mathrm{mg})$.

mp.: 219.5-220.0 ${ }^{\circ} \mathrm{C}, \mathrm{R}_{f}=0.190(\mathrm{DCM}: \mathrm{Hexs}=1: 1)$

${ }^{1} \mathbf{H}-\mathbf{N M R}\left(400 \mathrm{MHz}, \mathrm{CDCl} 3,25^{\circ} \mathrm{C}\right) \delta / \mathrm{ppm}: 8.34(\mathrm{~d}, 2 \mathrm{H}, J=8.7 \mathrm{~Hz}), 8.13(\mathrm{~d}, 2 \mathrm{H}, J=$ 9.0 Hz), 7.94-7.92 (m, 2H), $7.59(\mathrm{~d}, 2 \mathrm{H}, J=8.7 \mathrm{~Hz}), 7.52-7.50(\mathrm{~m}, 5 \mathrm{H}), 4.10(\mathrm{q}, 2 \mathrm{H}$, $J=7.1 \mathrm{~Hz}), 1.00(\mathrm{t}, 3 \mathrm{H}, J=7.1 \mathrm{~Hz})$.

${ }^{13}$ C-NMR $\left(100 \mathrm{MHz}, \mathrm{CDCl} 3,25{ }^{\circ} \mathrm{C}\right) \delta / \mathrm{ppm}: 162.9,157.3,147.8,147.0,146.4,139.7$, 135.1, 131.0, 130.2, 128.8, 128.5, 128.4, 126.1, 125.0, 124.1, 124.0, 116.7, 61.0, 13.6. MS (20 eV, EI) m/z (\%): $458[\mathrm{M}]^{+}(100), 431(26)$.

IR $(\mathrm{KBr}) \tilde{v}\left(\mathrm{~cm}^{-1}\right):$ 2989, 1721, 1596, 1520, 1344, 1232, 1110, 851, 693.

HRMS (EI) for $\mathbf{C}_{25} \mathbf{H}_{18} \mathbf{N}_{2} \mathbf{O}_{7},[\mathbf{M}]^{+}$(458.1114) found: 458.1107

\section{Synthesis of ethyl ethyl 3-(4-nitrophenyl)-5-phenyl-[2,2'-bifuran]-4-carboxylate} (7aj)

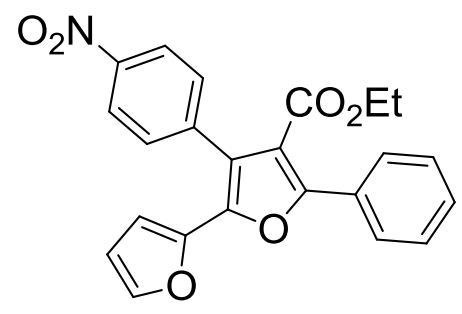

7aj

Prepared according to TP-A using $\mathbf{6 a}(97.6 \mathrm{mg}, 0.3 \mathrm{mmol}), 3$ (5.8 mg, $0.03 \mathrm{mmol})$, $\mathrm{Et}_{3} \mathrm{~N}(8.4 \mu \mathrm{L}, 0.06 \mathrm{mmol}), \mathrm{PhSiH}_{3}(61 \mu \mathrm{L}, 0.48 \mathrm{mmol}), 4 \mathbf{b}$ (10 $\left.\mu \mathrm{L}, 0.06 \mathrm{mmol}\right), 2 \mathbf{j}$ (27 $\mu \mathrm{L}, 0.33 \mathrm{mmol})$ and THF $(1.5 \mathrm{~mL})$. The reaction mixture was stirred for 15 hours at $50{ }^{\circ} \mathrm{C}$. Then the solvent was removed by evaporation in vacuo, and crude mixture was 
purified by flash column chromatography (DCM:Hexs $=1: 5)$ to provide 7aj as a yellow solid in $76 \%$ yield $(63.8 \mathrm{mg})$.

$\mathrm{mp}: 163.2-164.0{ }^{\circ} \mathrm{C}, \mathrm{R}_{f}=0.075(\mathrm{DCM}: \mathrm{Hexs}=1: 5)$

${ }^{1} \mathbf{H}$ NMR $\left(400 \mathrm{MHz}, \mathrm{CDCl} 3,25{ }^{\circ} \mathrm{C}\right) \delta / \mathrm{ppm}: 8.28(\mathrm{~d}, 2 \mathrm{H}, J=8.8 \mathrm{~Hz}), 7.90(\mathrm{~d}, 2 \mathrm{H}, J=$ $8.0 \mathrm{~Hz}), 7.60(\mathrm{~m}, 2 \mathrm{H}, J=8.4 \mathrm{~Hz}), 7.51-7.41(\mathrm{~m}, 3 \mathrm{H}), 7.34(\mathrm{~s}, 1 \mathrm{H}), 6.46-6.37(\mathrm{~m}, 2 \mathrm{H})$, $4.10(\mathrm{q}, 2 \mathrm{H}, J=7.2 \mathrm{~Hz}), 1.00(\mathrm{t}, 3 \mathrm{H}, J=7.1 \mathrm{~Hz})$.

${ }^{13} \mathrm{C}$ NMR (100 MHz, $\left.\mathrm{CDCl} 3,25{ }^{\circ} \mathrm{C}\right) \delta / \mathrm{ppm}: 163.3,155.8,147.3,144.5,142.9,141.9$, 139.5, 131.1, 129.7, 129.1, 128.3, 128.2, 123.1, 120.9, 115.5, 111.4, 108.6, 60.8, 13.6. IR $(\mathrm{KBr}) \tilde{v}\left(\mathrm{~cm}^{-1}\right): 1717,1517,1345,1234,1095,853,735,691$.

HRMS (EI) for $\mathbf{C}_{23} \mathbf{H}_{\mathbf{1 7}} \mathbf{N O}_{\mathbf{6}},[\mathbf{M}]^{+}$(403.1056) found: 403.1057 .

\section{Synthesis of ethyl 5-(4-chlorophenyl)-4-(4-cyanophenyl)-2-phenylfuran-3-} carboxylate $(7 \mathbf{b a})^{[\mathrm{a}]}$

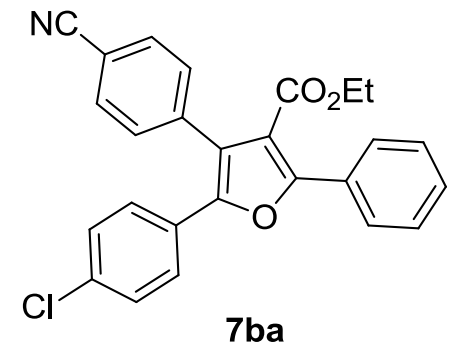

Prepared according to TP-A using $\mathbf{6 b}(91.6 \mathrm{mg}, 0.3 \mathrm{mmol}), 3(5.8 \mathrm{mg}, 0.03 \mathrm{mmol})$, $\mathrm{Et}_{3} \mathrm{~N}(8.4 \mu \mathrm{L}, 0.06 \mathrm{mmol}), \mathrm{PhSiH}_{3}(61 \mu \mathrm{L}, 0.48 \mathrm{mmol}), \mathbf{4 b}(10 \mu \mathrm{L}, 0.06 \mathrm{mmol})$ and $\mathbf{2 a}$ $(42 \mu \mathrm{L}, 0.33 \mathrm{mmol})$ in THF $(1.5 \mathrm{~mL})$. The reaction mixture was stirred for 10 hours at $50{ }^{\circ} \mathrm{C}$. Then the solvent was removed by evaporation in vacuo and the crude mixture was purified by flash column chromatography $(\mathrm{DCM}$ :Hexanes $=1: 4)$ to provide $7 \mathbf{b a}$ as a white solid in $90 \%$ yield $(115.5 \mathrm{mg})$

${ }^{1} \mathbf{H}$ NMR $\left(400 \mathrm{MHz}, \mathrm{CDCl}_{3}, 25{ }^{\circ} \mathrm{C}\right) \delta / \mathrm{ppm}: 7.90(\mathrm{~d}, 2 \mathrm{H}, J=7.1 \mathrm{~Hz}), 7.71(\mathrm{~d}, 2 \mathrm{H} J=$ $8.0 \mathrm{~Hz}), 7.56-7.40(\mathrm{~m}, 5 \mathrm{H}), 7.32-7.20(\mathrm{~m}, 4 \mathrm{H}), 4.08(\mathrm{q}, 2 \mathrm{H}, J=7.2 \mathrm{~Hz}), 0.98(\mathrm{t}, 3 \mathrm{H}, J$ $=7.2 \mathrm{~Hz})$ 
${ }^{13} \mathrm{C}$ NMR (100 MHz, $\left.\mathrm{CDCl}_{3}, 25{ }^{\circ} \mathrm{C}\right) \delta / \mathrm{ppm:} \mathrm{163.3,} \mathrm{155.8,} \mathrm{147.7,} \mathrm{138.3,} \mathrm{134.3,} \mathrm{132.2,}$ $130.9,129.6,129.2,128.9,128.3,128.1,127.7,127.1,122.2,118.6,116.1,111.7$ $60.7,13.5$.

\section{Synthesis of}

(5-(4-chlorophenyl)-4-(4-nitrophenyl)-2-phenylfuran-3-yl)(phenyl)methanone $(9 \mathbf{a a})^{[\mathrm{a}]}$

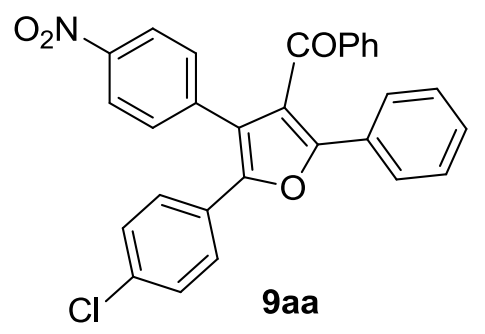

Prepared according to TP-A using $8 \mathbf{a}(107.2 \mathrm{mg}, 0.3 \mathrm{mmol}), \mathbf{3}$ (5.8 mg, $0.03 \mathrm{mmol})$, $\mathrm{Et}_{3} \mathrm{~N}(8.4 \mu \mathrm{L}, 0.06 \mathrm{mmol}), \mathrm{PhSiH}_{3}(61 \mu \mathrm{L}, 0.48 \mathrm{mmol}), \mathbf{4 b}(10 \mu \mathrm{L}, 0.06 \mathrm{mmol})$ and $\mathbf{2 a}$ $(42 \mu \mathrm{L}, 0.33 \mathrm{mmol})$ in THF $(1.5 \mathrm{~mL})$. The reaction mixture was stirred for 10 hours at $50{ }^{\circ} \mathrm{C}$. Then the solvent was removed by evaporation in vacuo, and the crude mixture was purified by flash column chromatography (DCM: Hexanes $=1: 4$ ) to provide 9aa as a yellow solid in $92 \%$ yield $(132.2 \mathrm{mg})$.

${ }^{1} \mathbf{H}$ NMR $\left(400 \mathrm{MHz}, \mathrm{CDCl}_{3}, 25{ }^{\circ} \mathrm{C}\right) \delta / \mathrm{ppm}: 8.11(\mathrm{~d}, 2 \mathrm{H}, J=8.5 \mathrm{~Hz}), 7.81(\mathrm{~d}, 2 \mathrm{H}, J=$ $7.9 \mathrm{~Hz}), 7.62-7.55(\mathrm{~m}, 2 \mathrm{H}), 7.49-7.38(\mathrm{~m}, 3 \mathrm{H}), 7.41(\mathrm{~d}, 2 \mathrm{H}, J=8.7 \mathrm{~Hz}), 7.34-7.26(\mathrm{~m}$, $7 \mathrm{H})$.

${ }^{13} \mathrm{C}$ NMR (100 MHz, $\left.\mathrm{CDCl}_{3}, 25{ }^{\circ} \mathrm{C}\right) \delta / \mathrm{ppm}: 192.4,152.4,148.1,147.3,139.1,137.0$, 134.6, 133.8, 130.7, 129.7, 129.1, 128.9, 128.7, 128.6, 127.8, 127.6, 126.6, 124.0, 123.0, 122.4

\section{Synthesis of}

(5-(3-chlorophenyl)-4-(4-nitrophenyl)-2-phenylfuran-3-yl)(phenyl)methanone 
(9ab)

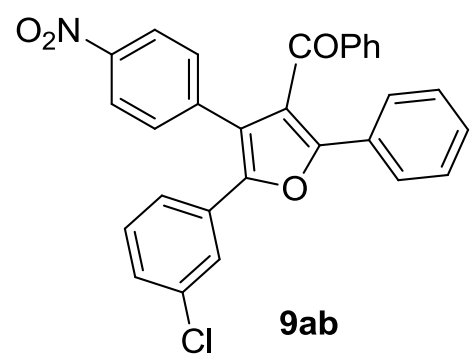

Prepared according to TP-A using $\mathbf{8 a}(107.2 \mathrm{mg}, 0.3 \mathrm{mmol}), \mathbf{3}$ (5.8 mg, $0.03 \mathrm{mmol})$, $\mathrm{Et}_{3} \mathrm{~N}(8.4 \mu \mathrm{L}, 0.06 \mathrm{mmol}), \mathrm{PhSiH}_{3}(61 \mu \mathrm{L}, 0.48 \mathrm{mmol}), 4 \mathbf{b}(10 \mu \mathrm{L}, 0.06 \mathrm{mmol})$ and 2b $(43 \mu \mathrm{L}, 0.33 \mathrm{mmol})$ in THF $(1.5 \mathrm{~mL})$. The reaction mixture was stirred for 10 hours at $50{ }^{\circ} \mathrm{C}$. Then the solvent was removed by evaporation in vacuo, and the crude mixture was purified by flash column chromatography (DCM: Hexanes $=1: 4)$ to provide 9ab as a yellow solid in $97 \%$ yield (139.4 $\mathrm{mg})$.

mp: $175.5-176.2{ }^{\circ} \mathrm{C} ; \mathrm{R}_{f}=0.125$ (dichloromethane $:$ Hexanes $=1: 4$ )

${ }^{1} \mathbf{H}$ NMR $\left(400 \mathrm{MHz}, \mathrm{CDCl}_{3}, 25{ }^{\circ} \mathrm{C}\right) \delta / \mathrm{ppm}: 8.12(\mathrm{~d}, 2 \mathrm{H}, J=8.7 \mathrm{~Hz}), 7.80(\mathrm{~d}, 2 \mathrm{H}, J=$ 7.5 Hz), 7.64-7.54 (m, 3H), 7.49-7.39 (m, 3H), 7.35-7.18 (m, 8H).

${ }^{13} \mathrm{C}$ NMR (100 MHz, $\left.\mathrm{CDCl}_{3}, 25{ }^{\circ} \mathrm{C}\right) \delta / \mathrm{ppm}: 192.3,152.6,147.6,147.3,138.9,137.0$, $134.9,133.8,131.0,130.7,130.0,129.7,129.2,128.8,128.7,128.63,128.60,126.7$, $126.3,124.3,123.9,123.0,122.9$.

IR $(\mathrm{KBr}) \tilde{v}\left(\mathrm{~cm}^{-1}\right): 1661,1597,1519,11343,693$

HRMS (EI) for $\mathbf{C}_{29} \mathbf{H}_{18} \mathbf{C I N O}_{4},[\mathbf{M}]^{+}$(479.0924) found: 479.0923

\section{Synthesis of}

(5-(2-chlorophenyl)-4-(4-nitrophenyl)-2-phenylfuran-3-yl)(phenyl)methanone

(9ac)

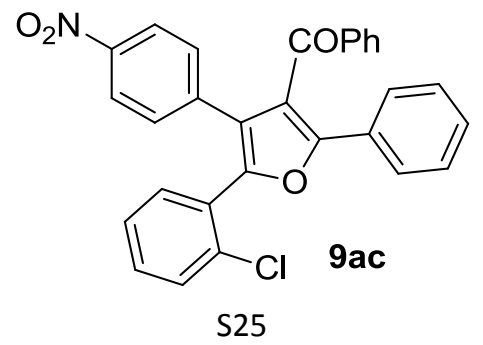


Prepared according to TP-A using $8 \mathbf{a}(107.2 \mathrm{mg}, 0.3 \mathrm{mmol}), \mathbf{3}(5.8 \mathrm{mg}, 0.03 \mathrm{mmol})$, $\mathrm{Et}_{3} \mathrm{~N}(8.4 \mu \mathrm{L}, 0.06 \mathrm{mmol}), \mathrm{PhSiH}_{3}(61 \mu \mathrm{L}, 0.48 \mathrm{mmol}), \mathbf{4 b}(10 \mu \mathrm{L}, 0.06 \mathrm{mmol})$ and $2 \mathbf{c}$ $(42 \mu \mathrm{L}, 0.33 \mathrm{mmol})$ in THF $(1.5 \mathrm{~mL})$. The reaction mixture was stirred for 10 hours at $50{ }^{\circ} \mathrm{C}$. Then the solvent was removed by evaporation in vacuo, and the crude mixture was purified by flash column chromatography (DCM: Hexanes $=1: 4)$ to provide 9ac as a pale yellow solid in $94 \%$ yield $(135.1 \mathrm{mg})$.

mp: $77-77.3{ }^{\circ} \mathrm{C} ; \mathrm{R}_{f}=0.1$ (dichloromethane : Hexanes $=1: 4$ )

${ }^{1} \mathbf{H}$ NMR $\left(400 \mathrm{MHz}, \mathrm{CDCl}_{3}, 25{ }^{\circ} \mathrm{C}\right) \delta / \mathrm{ppm}: 8.12(\mathrm{~d}, 2 \mathrm{H}, J=8.7 \mathrm{~Hz}), 7.80(\mathrm{~d}, 2 \mathrm{H}, J=$ 7.5 Hz), 7.64-7.54 (m, 3H), 7.49-7.39 (m, 3H), 7.35-7.18 (m, 8H).

${ }^{13} \mathrm{C}$ NMR (100 MHz, $\left.\mathrm{CDCl}_{3}, 25{ }^{\circ} \mathrm{C}\right) \delta / \mathrm{ppm}: 192.3,152.6,147.6,147.3,138.9,137.0$, $134.9,133.8,131.0,130.7,130.0,129.7,129.2,128.8,128.7,128.63,128.60,126.7$, $126.3,124.3,123.9,123.0,122.9$.

IR $(\mathrm{KBr}) \tilde{v}\left(\mathrm{~cm}^{-1}\right): 1661,1598,1519,1341,692$

HRMS (EI) for $\mathbf{C}_{29} \mathbf{H}_{18} \mathbf{C I N O}_{4}$, [M] ${ }^{+}$(479.0924) found: 479.0925.

\section{Synthesis of}

(5-(4-methoxyphenyl)-4-(4-nitrophenyl)-2-phenylfuran-3-yl)(phenyl)methanone $(\mathbf{9 a d})^{[\mathrm{a}]}$

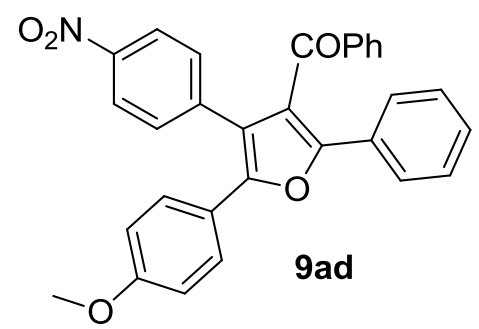

Prepared according to TP-A using $8 \mathbf{8}(107.2 \mathrm{mg}, 0.3 \mathrm{mmol}), 3$ (5.8 mg, $0.03 \mathrm{mmol})$, $\mathrm{Et}_{3} \mathrm{~N}(8.4 \mu \mathrm{L}, 0.06 \mathrm{mmol}), \mathrm{PhSiH}_{3}(61 \mu \mathrm{L}, 0.48 \mathrm{mmol}), \mathbf{4 b}(10 \mu \mathrm{L}, 0.06 \mathrm{mmol})$ and 2d $(46 \mu \mathrm{L}, 0.33 \mathrm{mmol})$ in $\mathrm{THF}(1.5 \mathrm{~mL})$. The reaction mixture was stirred for 10 hours at $50{ }^{\circ} \mathrm{C}$. Then the solvent was removed by evaporation in vacuo, and the crude 
mixture was purified by flash column chromatography (DCM: Hexanes $=1: 4)$ to provide 9ad as a yellow solid in 92\% yield $(131.1 \mathrm{mg})$

${ }^{1} \mathbf{H}$ NMR $\left(400 \mathrm{MHz}, \mathrm{CDCl}_{3}, 25{ }^{\circ} \mathrm{C}\right) \delta / \mathrm{ppm}: 8.09(\mathrm{~d}, 2 \mathrm{H}, J=8.1 \mathrm{~Hz}), 7.82(\mathrm{~d}, 2 \mathrm{H}, J=$ $7.3 \mathrm{~Hz}), 7.58(\mathrm{~d}, 2 \mathrm{H}, J=7.7 \mathrm{~Hz}), 7.48-7.37(\mathrm{~m}, 5 \mathrm{H}), 7.34-7.23(\mathrm{~m}, 5 \mathrm{H}), 6.86(\mathrm{~d}, 2 \mathrm{H}, J$ $=8.8 \mathrm{~Hz}), 3.82(\mathrm{~s}, 3 \mathrm{H})$.

${ }^{13} \mathrm{C}$ NMR (100 MHz, $\left.\mathrm{CDCl}_{3}, 25{ }^{\circ} \mathrm{C}\right) \delta / \mathrm{ppm}: 192.8,160.0,151.7,149.5,147.0,139.8$, $137.2,133.7,130.7,129.7,129.2,128.7,128.6,128.5,128.1,126.5,123.8,122.8$, $121.9,114.3,55.3$

Synthesis of (4-(4-nitrophenyl)-2,5-diphenylfuran-3-yl)(phenyl)methanone (9af) ${ }^{[\mathrm{a}]}$

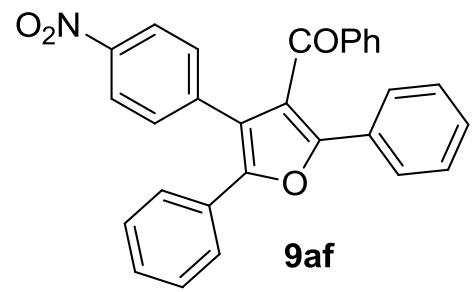

Prepared according to TP-A using $\mathbf{8 a}(107.2 \mathrm{mg}, 0.3 \mathrm{mmol}), 3$ (5.8 mg, $0.03 \mathrm{mmol})$, $\mathrm{Et}_{3} \mathrm{~N}(8.4 \mu \mathrm{L}, 0.06 \mathrm{mmol}), \mathrm{PhSiH}_{3}(61 \mu \mathrm{L}, 0.48 \mathrm{mmol}), \mathbf{4 b}(10 \mu \mathrm{L}, 0.06 \mathrm{mmol})$ and $\mathbf{2 f}$ $(34 \mu \mathrm{L}, 0.33 \mathrm{mmol})$ in THF $(1.5 \mathrm{~mL})$. The reaction mixture was stirred for 10 hours at $50{ }^{\circ} \mathrm{C}$. Then the solvent was removed by evaporation in vacuo, and the crude mixture was purified by flash column chromatography (DCM: Hexanes =1:4) to provide 9af as a yellow solid in $91 \%$ yield $(121.5 \mathrm{mg})$.

${ }^{1} \mathbf{H}$ NMR $\left(400 \mathrm{MHz}, \mathrm{CDCl}_{3}, 25{ }^{\circ} \mathrm{C}\right) \delta / \mathrm{ppm}: 8.10(\mathrm{~d}, 2 \mathrm{H}, J=8.5 \mathrm{~Hz}), 7.82(\mathrm{~d}, 2 \mathrm{H}, J=$ 7.8 Hz), 7.60-7.56 (m, 2H), 7.54-7.40 (m, 5H), 7.38-7.26 (m, 8H).

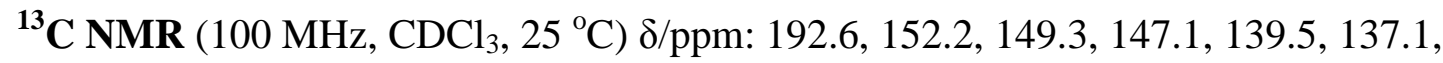
$133.8,130.7,129.7,129.3,129.0,128.9,128.8,128.7,128.6,128.5,126.6,126.5$, $123.8,122.9,122.0$ 
Synthesis of (4,5-bis(4-nitrophenyl)-2-phenylfuran-3-yl)(phenyl)methanone

(9ai) ${ }^{[\mathrm{a}]}$

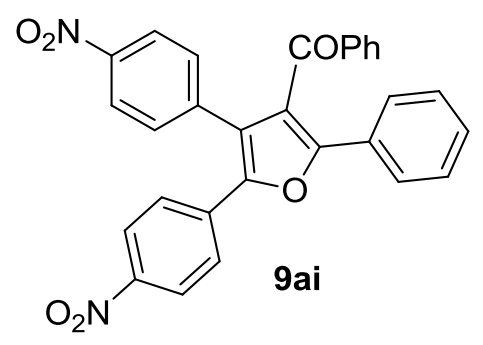

Prepared according to TP-A using $8 \mathbf{a}(107.2 \mathrm{mg}, 0.3 \mathrm{mmol}), \mathbf{3}$ (5.8 mg, $0.03 \mathrm{mmol})$, $\mathrm{Et}_{3} \mathrm{~N}(8.4 \mu \mathrm{L}, 0.06 \mathrm{mmol}), \mathrm{PhSiH}_{3}(61 \mu \mathrm{L}, 0.48 \mathrm{mmol}), \mathbf{4 b}(10 \mu \mathrm{L}, 0.06 \mathrm{mmol})$ and $2 \mathbf{i}$ (63 mg, $0.33 \mathrm{mmol})$ in THF $(1.5 \mathrm{~mL})$. The reaction mixture was stirred for 10 hours at $50{ }^{\circ} \mathrm{C}$. Then the solvent was removed by evaporation in vacuo, and the crude mixture was purified by flash column chromatography (DCM: Hexanes $=1: 4)$ to provide 9ai as a yellow solid in $89 \%$ yield $(130.9 \mathrm{mg})$.

${ }^{1} \mathbf{H}$ NMR $\left(400 \mathrm{MHz}, \mathrm{CDCl}_{3}, 25{ }^{\circ} \mathrm{C}\right) \delta / \mathrm{ppm}: 8.22-8.14(\mathrm{~m}, 4 \mathrm{H}), 7.81(\mathrm{~d}, 2 \mathrm{H}, J=7.5$ $\mathrm{Hz})$, 7.66-7.58 (m, 4H), 7.54-7.41 (m, 3H), 7.37-7.27 (m, 5H).

${ }^{13} \mathrm{C}$ NMR (100 MHz, $\left.\mathrm{CDCl}_{3}, 25{ }^{\circ} \mathrm{C}\right) \delta / \mathrm{ppm}: 191.9,153.6,147.7,147.0,146.5,138.4$, $136.8,135.1,134.0,130.7,129.7,129.6,128.8,128.7,128.4,126.8,126.3,125.4$, 124.2, 123.6 .

Synthesis of (3-(4-nitrophenyl)-5-phenyl-[2,2'-bifuran]-4-yl)(phenyl)methanone $(\mathbf{9 a j})^{[\mathrm{a}]}$

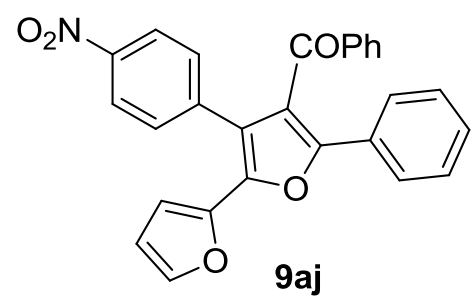

Prepared according to TP-A using $8 \mathbf{a}(107.2 \mathrm{mg}, 0.3 \mathrm{mmol}), 3$ (5.8 mg, $0.03 \mathrm{mmol})$, $\mathrm{Et}_{3} \mathrm{~N}(8.4 \mu \mathrm{L}, 0.06 \mathrm{mmol}), \mathrm{PhSiH}_{3}(61 \mu \mathrm{L}, 0.48 \mathrm{mmol}), \mathbf{4 b}(10 \mu \mathrm{L}, 0.06 \mathrm{mmol})$ and $\mathbf{2 j}$ 
$(27 \mu \mathrm{L}, 0.33 \mathrm{mmol})$ in THF $(1.5 \mathrm{~mL})$. The reaction mixture was stirred for 7 hours at $50{ }^{\circ} \mathrm{C}$. Then the solvent was removed by evaporation in vacuo, and the crude mixture was purified by flash column chromatography (DCM: Hexanes = 1:4) to provide 9aj as a yellow solid in $89 \%$ yield (116.2 $\mathrm{mg})$.

${ }^{1} \mathbf{H}$ NMR $\left(400 \mathrm{MHz}, \mathrm{CDCl}_{3}, 25{ }^{\circ} \mathrm{C}\right) \delta / \mathrm{ppm}: 8.11(\mathrm{~d}, 2 \mathrm{H}, J=8.6 \mathrm{~Hz}), 7.81(\mathrm{~d}, 2 \mathrm{H}, J=$ 7.7 Hz), 7.63-7.57 (m, 2H), $7.51(\mathrm{~d}, 2 \mathrm{H}, J=8.8 \mathrm{~Hz}), 7.44(\mathrm{t}, 1 \mathrm{H}, J=7.4 \mathrm{~Hz})$,

7.41-7.38 (m, 1H), 7.34-7.24 (m, 5H), $6.64(\mathrm{~d}, 1 \mathrm{H}, J=3.5 \mathrm{~Hz}), 6.48\left(\mathrm{dd}, 1 \mathrm{H}, J^{1}=3.5\right.$ $\left.\mathrm{Hz}, J^{2}=1.8 \mathrm{~Hz}\right)$

${ }^{13} \mathrm{C}$ NMR (100 MHz, $\left.\mathrm{CDCl}_{3}, 25{ }^{\circ} \mathrm{C}\right)$ d/ppm: 192.3, 152.3, 147.1, 144.8, 143.0, 141.7, $138.4,137.0,133.8,130.7,129.7,129.1,128.8,128.6,128.5,126.6,123.3,122.5$, 121.6, 111.6, 108.9.

\section{Synthesis of}

\section{(5-(4-chlorophenyl)-2-phenyl-4-(thiophen-2-yl)furan-3-yl)(phenyl)methanone}

(9ba)

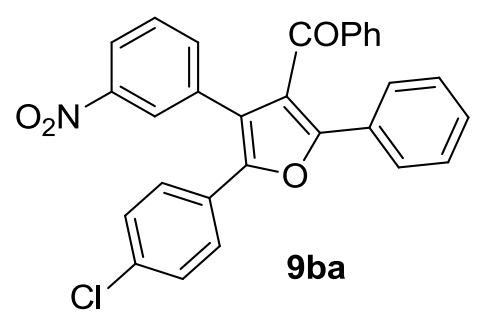

Prepared according to TP-A using $\mathbf{8 b}$ (107.2 mg, $0.3 \mathrm{mmol}), 3$ (5.8 mg, $0.03 \mathrm{mmol}$ ), $\mathrm{Et}_{3} \mathrm{~N}(8.4 \mu \mathrm{L}, 0.06 \mathrm{mmol}), \mathrm{PhSiH}_{3}(61 \mu \mathrm{L}, 0.48 \mathrm{mmol}), \mathbf{4 b}(10 \mu \mathrm{L}, 0.06 \mathrm{mmol})$ and $\mathbf{2 a}$ $(42 \mu \mathrm{L}, 0.33 \mathrm{mmol})$ in THF $(1.5 \mathrm{~mL})$. The reaction mixture was stirred for 36 hours at $50{ }^{\circ} \mathrm{C}$. Then the solvent was removed by evaporation in vacuo, and the crude mixture was purified by flash column chromatography (DCM: Hexanes $=1: 4)$ to provide 9 ba as a yellow solid in $97 \%$ yield $(139.4 \mathrm{mg})$.

mp: 164.4-164.5 ${ }^{\circ} \mathrm{C} ; \mathrm{R}_{f}: 0.13$ (dichloromethane : Hexanes = 1:4)

${ }^{1}$ H NMR $\left(400 \mathrm{MHz}, \mathrm{CDCl}_{3}, 25^{\circ} \mathrm{C}\right) \delta / \mathrm{ppm}: 8.15(\mathrm{~s}, 1 \mathrm{H}), 8.09(\mathrm{~d}, 1 \mathrm{H}, J=8.4 \mathrm{~Hz})$, 
$7.81(\mathrm{~d}, 2 \mathrm{H}, J=7.5 \mathrm{~Hz}), 7.67-7.56(\mathrm{~m}, 3 \mathrm{H}), 7.49-7.37(\mathrm{~m}, 4 \mathrm{H}), 7.34-7.22(\mathrm{~m}, 7 \mathrm{H})$.

${ }^{13} \mathrm{C}$ NMR (100 MHz, $\left.\mathrm{CDCl}_{3}, 25{ }^{\circ} \mathrm{C}\right) \delta / \mathrm{ppm:} \mathrm{192.4,} \mathrm{152.4,} \mathrm{148.3,} \mathrm{148.0,} \mathrm{137.1,} \mathrm{136.1,}$ $134.4,133.9,133.7,129.7,129.6,129.1,128.9,128.6,128.5,127.7,127.2,126.7$, $124.8,123.2,122.8,122.1$

IR $(\mathrm{KBr}) \tilde{v}\left(\mathrm{~cm}^{-1}\right): 1660,1530,1493,1348,69$

HRMS (EI) for $\mathbf{C}_{29} \mathbf{H}_{18} \mathrm{CINO}_{4},[\mathbf{M}]^{+}$(479.0924) found: 479.0925

\section{Synthesis of}

(5-(4-chlorophenyl)-4-(2-nitrophenyl)-2-phenylfuran-3-yl)(phenyl)methanone (9ca)

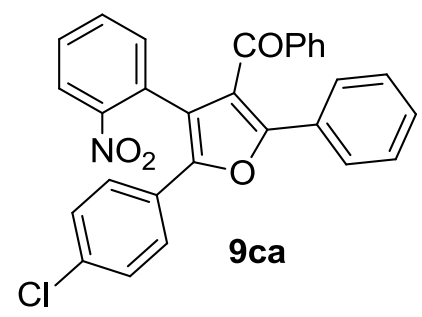

Prepared according to TP-A using $\mathbf{8 c}(107.2 \mathrm{mg}, 0.3 \mathrm{mmol}), \mathbf{3}$ (5.8 mg, $0.03 \mathrm{mmol})$, $\mathrm{Et}_{3} \mathrm{~N}(8.4 \mu \mathrm{L}, 0.06 \mathrm{mmol}), \mathrm{PhSiH}_{3}(61 \mu \mathrm{L}, 0.48 \mathrm{mmol}), \mathbf{4 b}(10 \mu \mathrm{L}, 0.06 \mathrm{mmol})$ and $\mathbf{2 a}$ $(42 \mu \mathrm{L}, 0.33 \mathrm{mmol})$ in THF $(1.5 \mathrm{~mL})$. The reaction mixture was stirred for 10 hours at $50{ }^{\circ} \mathrm{C}$. Then the solvent was removed by evaporation in vacuo, and crude mixture was purified by flash column chromatography (DCM: Hexanes $=1: 4)$ to provide 9ca as a yellow solid in $90 \%$ yield $(129.3 \mathrm{mg})$.

mp: $164.3-165.2{ }^{\circ} \mathrm{C} ; \mathrm{R}_{f}: 0.1$ (dichloromethane : Hexanes $=1: 4$ )

${ }^{1} \mathbf{H}$ NMR $\left(400 \mathrm{MHz}, \mathrm{CDCl}_{3}, 25{ }^{\circ} \mathrm{C}\right) \delta / \mathrm{ppm}: 8.03(\mathrm{~d}, 2 \mathrm{H}, J=8.4 \mathrm{~Hz}), 7.77(\mathrm{~d}, 2 \mathrm{H}, J=$ $8.8 \mathrm{~Hz}), 7.60(\mathrm{t}, 1 \mathrm{H}, J=7.1 \mathrm{~Hz}), 7.51(\mathrm{t}, 2 \mathrm{H}, J=8.0 \mathrm{~Hz}), 7.48-7.43(\mathrm{~m}, 2 \mathrm{H}), 7.35(\mathrm{t}$, $1 \mathrm{H}, J=7.5 \mathrm{~Hz}), 7.30(\mathrm{~d}, 2 \mathrm{H}, J=8.4 \mathrm{~Hz}), 7.24(\mathrm{~d}, 2 \mathrm{H}, J=8.8 \mathrm{~Hz}), 7.22-7.16(\mathrm{~m}, 4 \mathrm{H})$. ${ }^{13} \mathrm{C}$ NMR (100 MHz, $\left.\mathrm{CDCl}_{3}, 25{ }^{\circ} \mathrm{C}\right) \delta / \mathrm{ppm}: 191.8,153.6,149.0,147.8,136.9,134.1$, $133.4,133.3,133.1,129.7,129.4,128.9,128.9,128.3,128.2,127.9,127.5,127.5$, 
$126.7,124.8,123.2,120.5$.

IR $(\mathrm{KBr}) \tilde{v}\left(\mathrm{~cm}^{-1}\right): 1655,1650,1527,1494,1348,739,693$

HRMS (EI) for $\mathbf{C}_{29} \mathbf{H}_{18} \mathbf{C I N O}_{4},[\mathbf{M}]^{+}$(479.0924) found: 479.0925

\section{Synthesis of}

\section{(5-(4-chlorophenyl)-2-phenyl-4-(thiophen-2-yl)furan-3-yl)(phenyl)methanone}

(9da)

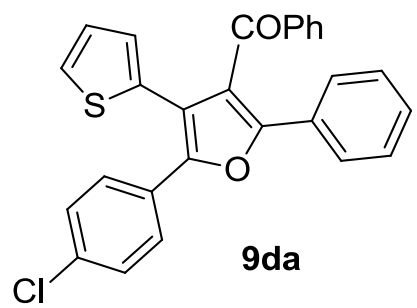

Prepared according to TP-A using $\mathbf{8 d}(97.5 \mathrm{mg}, 0.3 \mathrm{mmol}), 3(5.8 \mathrm{mg}, 0.03 \mathrm{mmol})$, $\mathrm{Et}_{3} \mathrm{~N}(8.4 \mu \mathrm{L}, 0.06 \mathrm{mmol}), \mathrm{PhSiH}_{3}(61 \mu \mathrm{L}, 0.48 \mathrm{mmol}), \mathbf{4 b}(10 \mu \mathrm{L}, 0.06 \mathrm{mmol})$ and $\mathbf{2 a}$ (42 $\mu \mathrm{L}, 0.33 \mathrm{mmol})$ in THF $(1.5 \mathrm{~mL})$. The reaction mixture was stirred for 24 hours at $50{ }^{\circ} \mathrm{C}$. Then the solvent was removed by evaporation in vacuo, and the crude mixture was purified by flash column chromatography $(\mathrm{DCM}$ : Hexanes $=1: 4)$ to provide 9da as a yellow solid in $85 \%$ yield $(112.4 \mathrm{mg})$.

mp: $141.0-141.8^{\circ} \mathrm{C} ; \mathrm{R}_{f}: 0.26$ (dichloromethane : Hexanes = 1:4)

${ }^{1} \mathbf{H}$ NMR $\left(400 \mathrm{MHz}, \mathrm{CDCl}_{3}, 25{ }^{\circ} \mathrm{C}\right) \delta / \mathrm{ppm}: 7.86(\mathrm{~d}, 2 \mathrm{H}, J=7.5 \mathrm{~Hz}), 7.60(\mathrm{~d}, 2 \mathrm{H}, J=$ $8.4 \mathrm{~Hz}), 7.54(\mathrm{~d}, 2 \mathrm{H}, J=8.8 \mathrm{~Hz}), 7.43(\mathrm{t}, 1 \mathrm{H}, J=7.5 \mathrm{~Hz}), 7.34-7.21(\mathrm{~m}, 7 \mathrm{H}), 7.20(\mathrm{~d}$, $1 \mathrm{H}, J=5.3 \mathrm{~Hz}), 6.94(\mathrm{~d}, 1 \mathrm{H}, J=3.5 \mathrm{~Hz}), 6.88\left(\mathrm{dd}, 1 \mathrm{H}, J^{1}=5.3 \mathrm{~Hz}, J^{2}=3.5 \mathrm{~Hz}\right)$.

${ }^{13} \mathbf{C}$ NMR $\left(100 \mathrm{MHz}, \mathrm{CDCl}_{3}, 25{ }^{\circ} \mathrm{C}\right) \delta / \mathrm{ppm}: 192.8,150.9,148.4,137.2,134.0,133.5$, $131.7,129.7,129.1,128.7,128.7,128.6,128.6,128.6,128.4,128.1,127.3,127.3$, $126.9,126.1,124.0,117.1$

IR $(\mathrm{KBr}) \tilde{v}\left(\mathrm{~cm}^{-1}\right): 1663,1483,1234,1098,691$

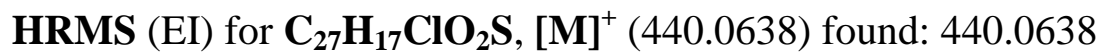




\section{Synthesis of ethyl 4-benzoyl-2-(4-chlorophenyl)-5-phenylfuran-3-carboxylate}

(9ea)

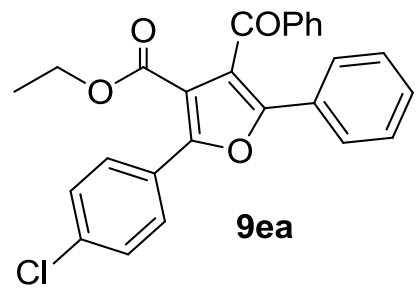

Prepared according to TP-A using $8 \mathrm{e}(92.5 \mathrm{mg}, 0.3 \mathrm{mmol}), 3(5.8 \mathrm{mg}, 0.03 \mathrm{mmol})$, $\mathrm{Et}_{3} \mathrm{~N}(8.4 \mu \mathrm{L}, 0.06 \mathrm{mmol}), \mathrm{PhSiH}_{3}(61 \mu \mathrm{L}, 0.48 \mathrm{mmol}), \mathbf{4 b}(10 \mu \mathrm{L}, 0.06 \mathrm{mmol})$ and $\mathbf{2 a}$ $(42 \mu \mathrm{L}, 0.33 \mathrm{mmol})$ in THF $(1.5 \mathrm{~mL})$. The reaction mixture was stirred for 7 hours at $50{ }^{\circ} \mathrm{C}$. Then the solvent was removed by evaporation in vacuo, and the crude mixture was purified by flash column chromatography (DCM: Hexanes $=1: 4)$ to provide 9ea as a white solid in $99 \%$ yield $(127.7 \mathrm{mg})$.

mp: 103.3-103.4 ${ }^{\circ} \mathrm{C} ; \mathrm{R}_{f}:$ 0.11(dichloromethane : Hexanes $\left.=1: 4\right)$

${ }^{1} \mathbf{H}$ NMR $\left(400 \mathrm{MHz}, \mathrm{CDCl}_{3}, 25{ }^{\circ} \mathrm{C}\right) \delta / \mathrm{ppm}: 8.04(\mathrm{~d}, 2 \mathrm{H}, J=8.8 \mathrm{~Hz}), 7.98(\mathrm{~d}, 2 \mathrm{H}, J=$ 7.1 Hz), 7.68-7.60 (m, 2H), 7.56 (t, 1H, J= 7.1 Hz), 7.50-7.40 (m, 4H), 7.36-7.25 (m, $3 \mathrm{H}), 3.96(\mathrm{q}, 2 \mathrm{H}, J=7.1 \mathrm{~Hz}), 0.87(\mathrm{t}, 3 \mathrm{H}, J=7.1 \mathrm{~Hz})$.

${ }^{13} \mathrm{C}$ NMR $\left(100 \mathrm{MHz}, \mathrm{CDCl}_{3}, 25{ }^{\circ} \mathrm{C}\right) \delta / \mathrm{ppm}: 192.0,162.3,154.7,150.4,137.5,135.8$ $133.6,129.7,129.3,129.0,128.7,128.7,128.6,128.5,127.3,126.0,122.0,115.8$, $61.0,13.2$.

IR $(\mathrm{KBr}) \tilde{v}\left(\mathrm{~cm}^{-1}\right): 3062,2982,1721,1673,1227,691$

HRMS (EI) for $\mathbf{C}_{26} \mathbf{H}_{19} \mathbf{C l O}_{4}$, [M] ${ }^{+}$(430.0972) found: 430.0970 .

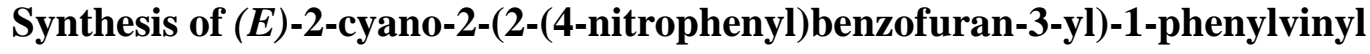
pivalate (10) ${ }^{[b]}$

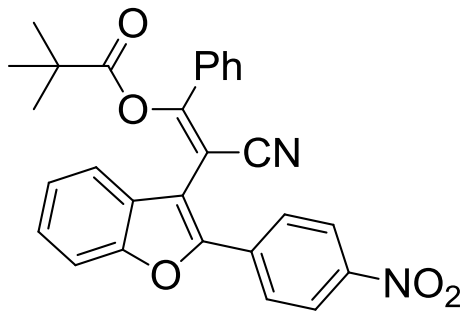


Prepared according to TP-A using $\mathbf{1 h}(119.5 \mathrm{mg}, 0.3 \mathrm{mmol}), \mathbf{3}$ (5.8 mg, $0.03 \mathrm{mmol})$, $\mathrm{Et}_{3} \mathrm{~N}(8.4 \mu \mathrm{L}, 0.06 \mathrm{mmol}), \mathrm{PhSiH}_{3}(61 \mu \mathrm{L}, 0.48 \mathrm{mmol}), \mathbf{4 b}(10 \mu \mathrm{L}, 0.06 \mathrm{mmol}), \mathbf{2 k}$ $(41 \mu \mathrm{L}, 0.33 \mathrm{mmol})$ and THF $(1.5 \mathrm{~mL})$. The reaction mixture was stirred for 15 hours at $50{ }^{\circ} \mathrm{C}$. Then the solvent was removed by evaporation in vacuo, and crude mixture was purified by flash column chromatography (DCM:Hexs $=1: 5$ ) to provide $\mathbf{1 0}$ as a yellow solid in $68 \%$ yield $(95.1 \mathrm{mg})$.

$\mathrm{mp}: 171.5-172.5^{\circ} \mathrm{C}, \mathrm{R}_{f}=0.100(\mathrm{DCM}: \mathrm{Hexs}=1: 5)$

${ }^{1} \mathbf{H}$ NMR $\left(400 \mathrm{MHz}, \mathrm{CDCl} 3,25{ }^{\circ} \mathrm{C}\right) \delta / \mathrm{ppm}: 8.37(\mathrm{~d}, 2 \mathrm{H}, J=8.8 \mathrm{~Hz}), 8.15(\mathrm{~d}, 2 \mathrm{H}, J=$ $8.8 \mathrm{~Hz}), 7.89(\mathrm{~d}, 2 \mathrm{H}, J=7.5 \mathrm{~Hz}), 7.36-7.52(\mathrm{~m}, 5 \mathrm{H}), 7.43(\mathrm{t}, 1 \mathrm{H}, J=7.7 \mathrm{~Hz}), 7.36(\mathrm{t}$, $1 \mathrm{H}, J=7.5 \mathrm{~Hz}), 0.81(\mathrm{~s}, 9 \mathrm{H})$.

${ }^{13} \mathrm{C}$ NMR (100 MHz, CDCl3, $\left.25^{\circ} \mathrm{C}\right) \delta / \mathrm{ppm}: 174.6,163.6,154.0,150.5,147.8,135.3$, $132.1,132.0,129.1,127.8,127.6,126.6,124.2,124.0,120.9,116.3,111.7,110.5$, 94.7, 39.1, 26.3.

HRMS (ESI) for $\mathbf{C}_{28} \mathbf{H}_{22} \mathbf{N}_{2} \mathbf{O}_{5} \mathbf{N a},[\mathbf{M}+\mathbf{N a}]^{+}$(489.1426) found: 489.1421.

\section{Synthesis of 2-(2-bromobenzyl)-3-oxo-3-phenylpropanenitrile (11):}<smiles>N#CC(Cc1ccccc1Br)C(=O)c1ccccc1</smiles>

11

Prepared according to TP-D using $\mathbf{1 a}(93.6 \mathrm{mg}, 0.3 \mathrm{mmol}), \mathrm{P}(\mathrm{O}) \mathrm{Bu}_{3}(6.5 \mathrm{mg}, 0.03$ mmol), $\mathrm{Et}_{3} \mathrm{~N}(8.4 \mu \mathrm{L}, 0.06 \mathrm{mmol}), \mathrm{PhSiH}_{3}(61 \mu \mathrm{L}, 0.48 \mathrm{mmol}), 4 \mathbf{b}(10 \mu \mathrm{L}, 0.06 \mathrm{mmol})$ and THF $(1.5 \mathrm{~mL})$. The reaction mixture was stirred for 24 hours at $50{ }^{\circ} \mathrm{C}$. Then the solvent was removed by evaporation in vacuo, and crude mixture was purified by flash column chromatography (EA:Hexs $=1: 27$ ) to provide $\mathbf{1 1}$ as a colorless liquid. $\mathrm{R}_{f}=0.4(\mathrm{EA}: \operatorname{Hexs}=1: 8)$ 
${ }^{1} \mathbf{H}$ NMR $\left(400 \mathrm{MHz}, \mathrm{CDCl} 3,25{ }^{\circ} \mathrm{C}\right) \delta / \mathrm{ppm}: 8.02(\mathrm{~d}, 2 \mathrm{H}, J=7.1 \mathrm{~Hz}), 7.65(\mathrm{t}, 1 \mathrm{H}, J=$ $7.5 \mathrm{~Hz}), 7.58(\mathrm{~d}, 1 \mathrm{H}, J=8.0 \mathrm{~Hz}), 7.52(\mathrm{t}, 2 \mathrm{H}, J=7.4 \mathrm{~Hz}), 7.41(\mathrm{~d}, 1 \mathrm{H}, J=7.5 \mathrm{~Hz})$, $7.31(\mathrm{t}, 1 \mathrm{H}, J=7.5 \mathrm{~Hz}), 7.17(\mathrm{t}, 1 \mathrm{H}, J=7.7 \mathrm{~Hz}), 4.81(\mathrm{dd}, 1 \mathrm{H}, J=9.6,6.0 \mathrm{~Hz}), 3.54$ $(\mathrm{dd}, 1 \mathrm{H}, J=13.9,6.0 \mathrm{~Hz}), 3.33-3.21(\mathrm{dd}, 1 \mathrm{H}, J=13.9,9.6 \mathrm{~Hz})$.

${ }^{13} \mathrm{C}$ NMR $\left(100 \mathrm{MHz}, \mathrm{CDCl}_{3}, 25{ }^{\circ} \mathrm{C}\right) \delta / \mathrm{ppm}: 190,135.2,134.7,134.1,133.1,132.3$, $129.6,129.1,128.9,128.1,124.2,116.6,39.2,36.0$.

IR $(\mathrm{KBr}) \tilde{v}\left(\mathrm{~cm}^{-1}\right):$ 3062, 2922, 1597, 1448, 1260, 1027, 752, 694.

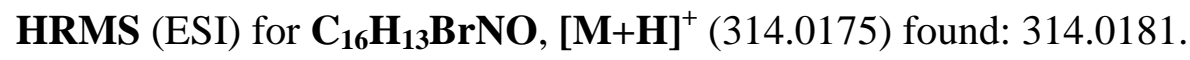

\section{REFERENCES}

[a] T.-T. Kao, S.-E. Syu, Y.-W. Jhang, W. Lin, Org. Lett. 2010, 12, 3066.

[b] Y.-T. Lee, Y.-T. Lee, C.-J. Lee, C.-N. Sheu, B.-Y. Lin, J.-H. Wang, W. Lin, Org. Biomol. Chem. 2013, 11, 5156. 
VI. ${ }^{1} \mathrm{H}$ NMR and ${ }^{13} \mathrm{C}$ NMR spectra of the products
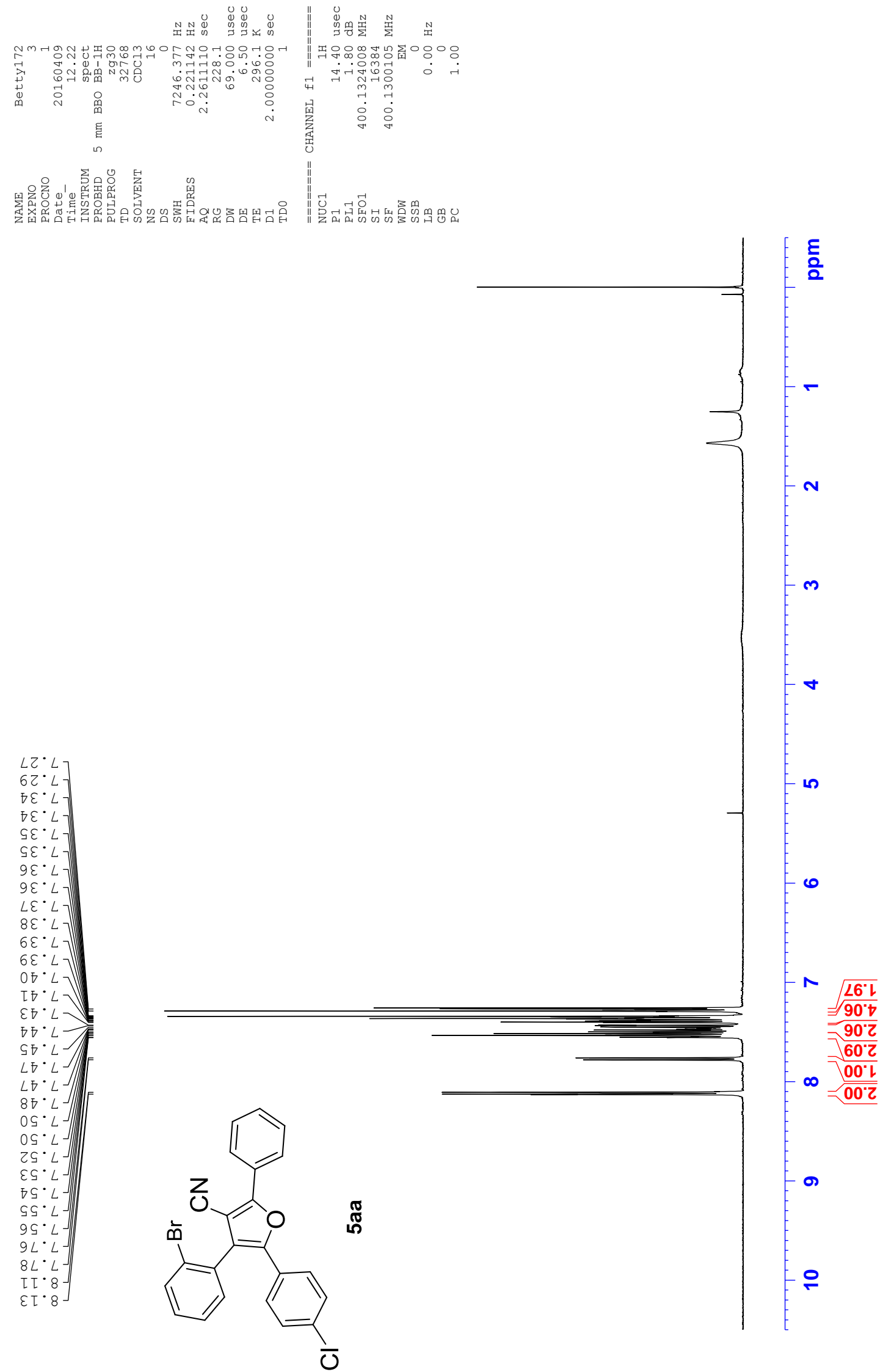

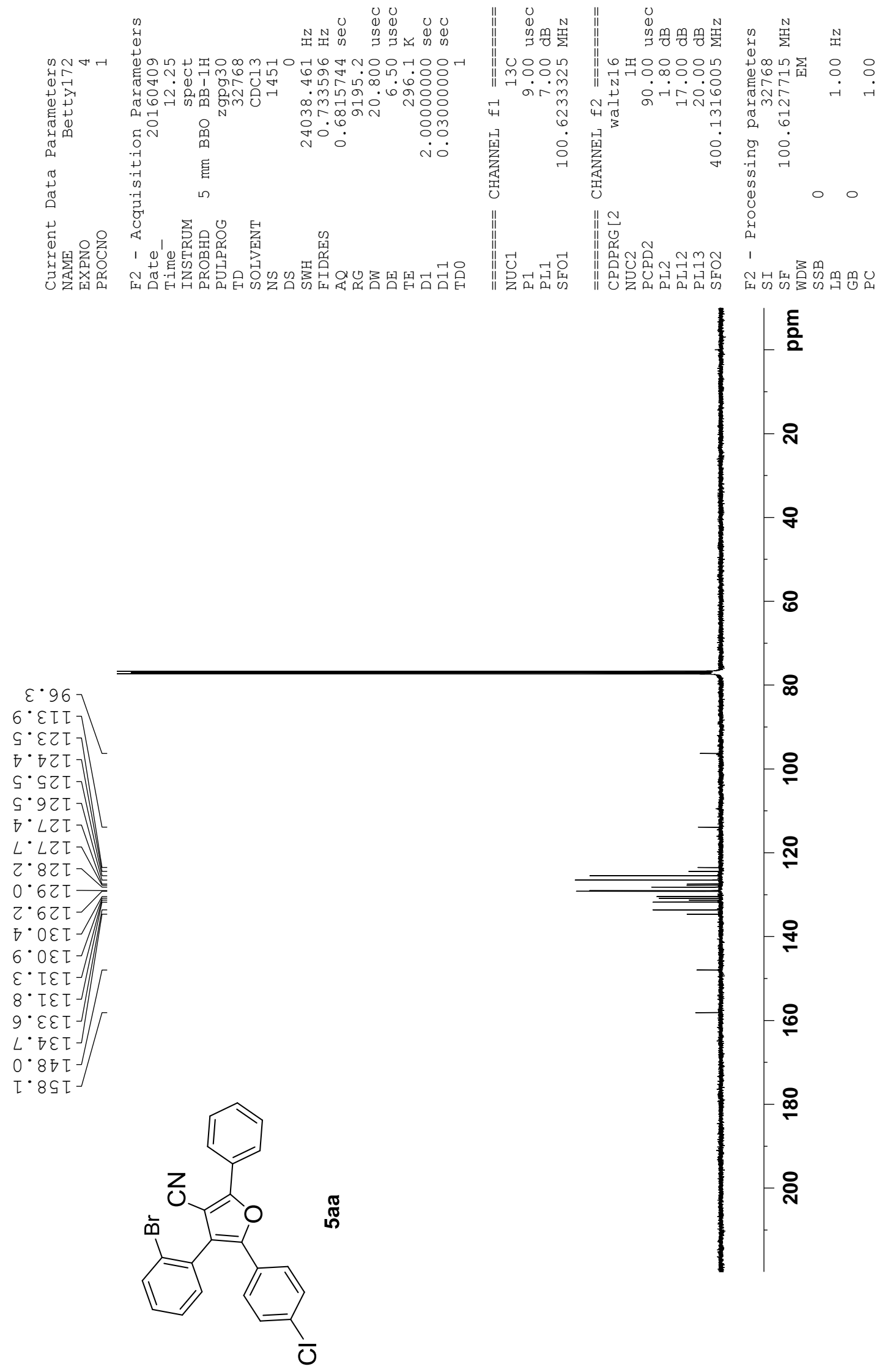

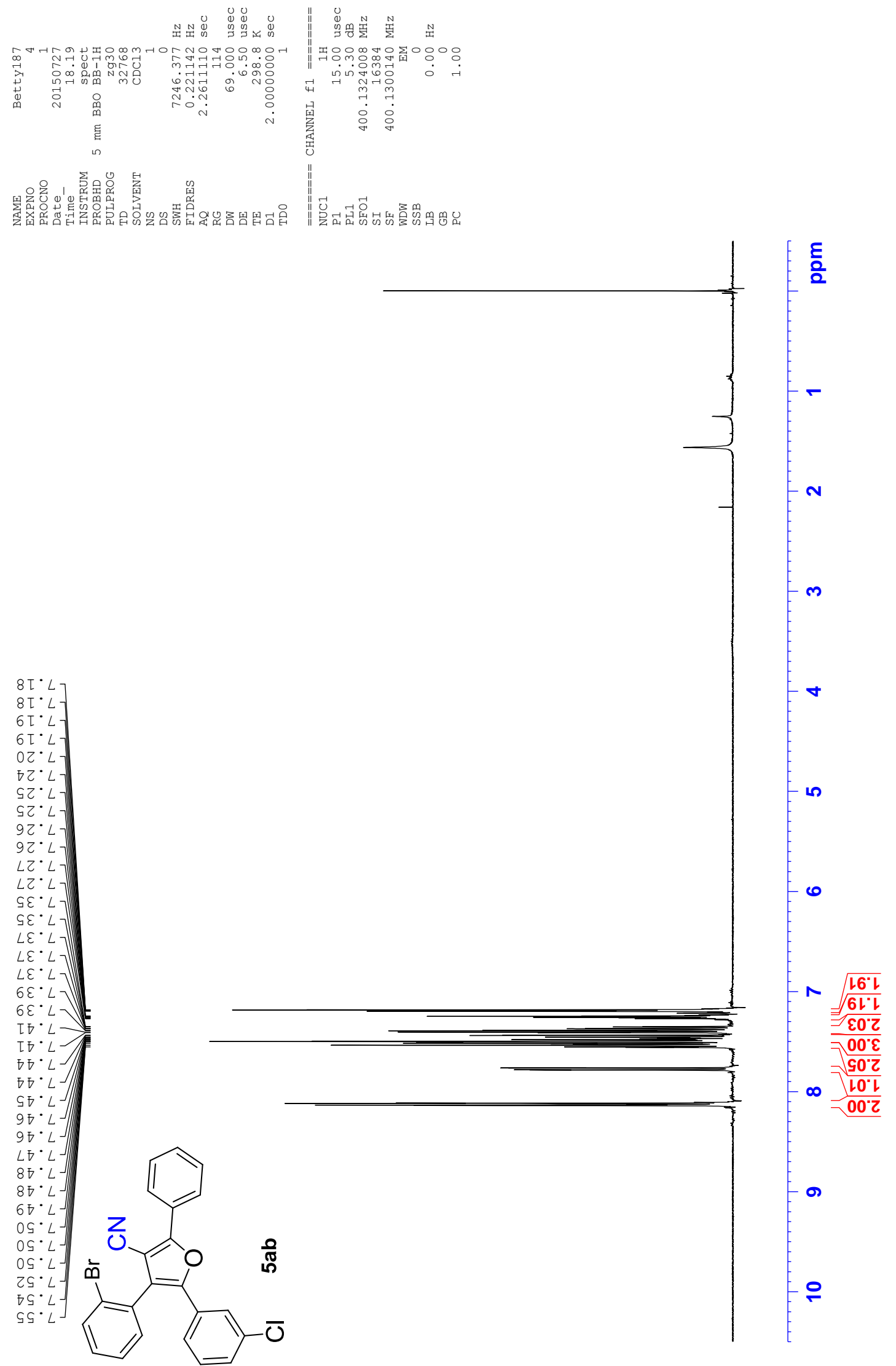

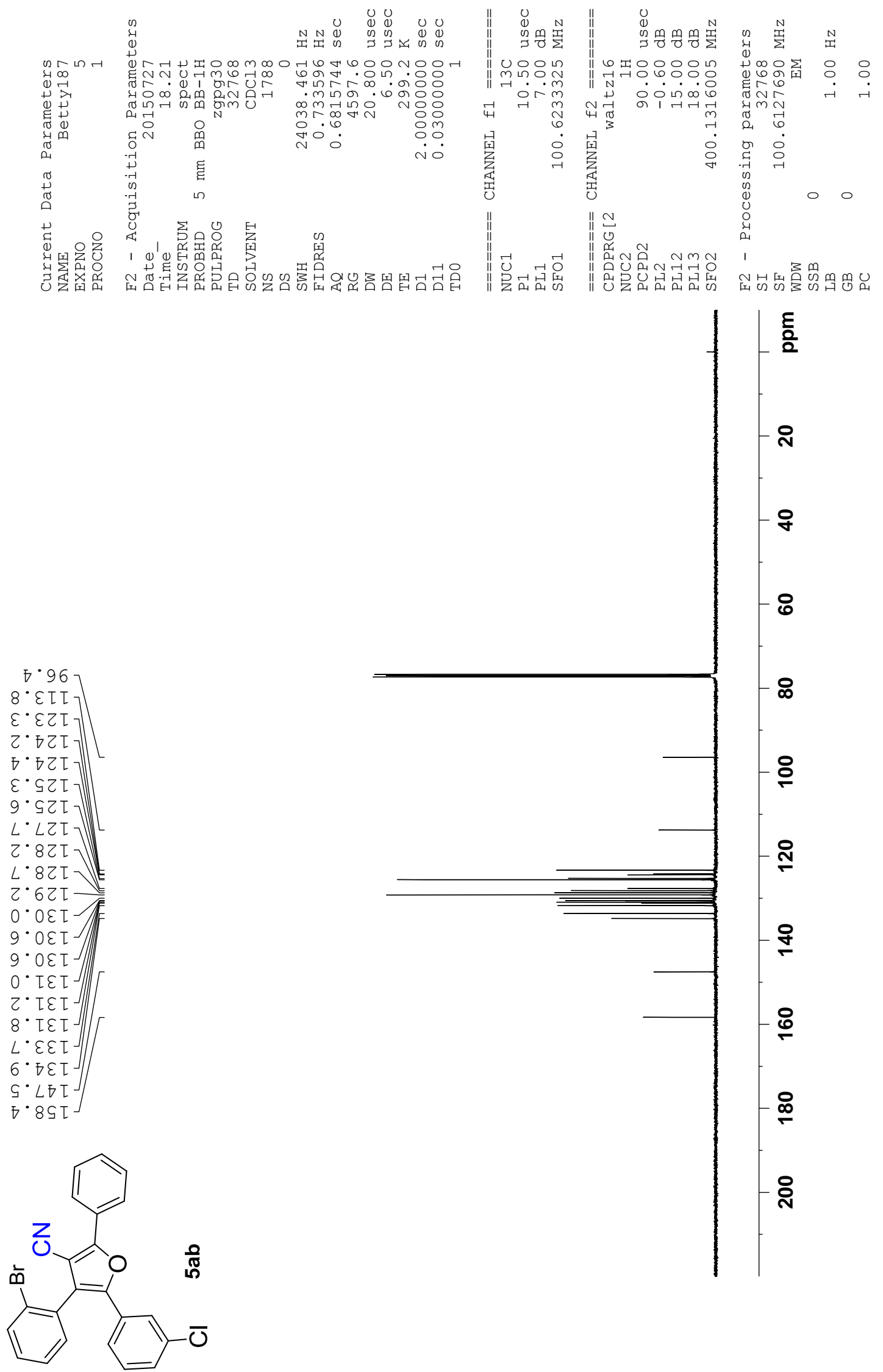

등

군

q

8

ㅇ

우

ิㅗ

q

$\stackrel{\circ}{-}$

$\stackrel{\circ}{\circ}$

จั 

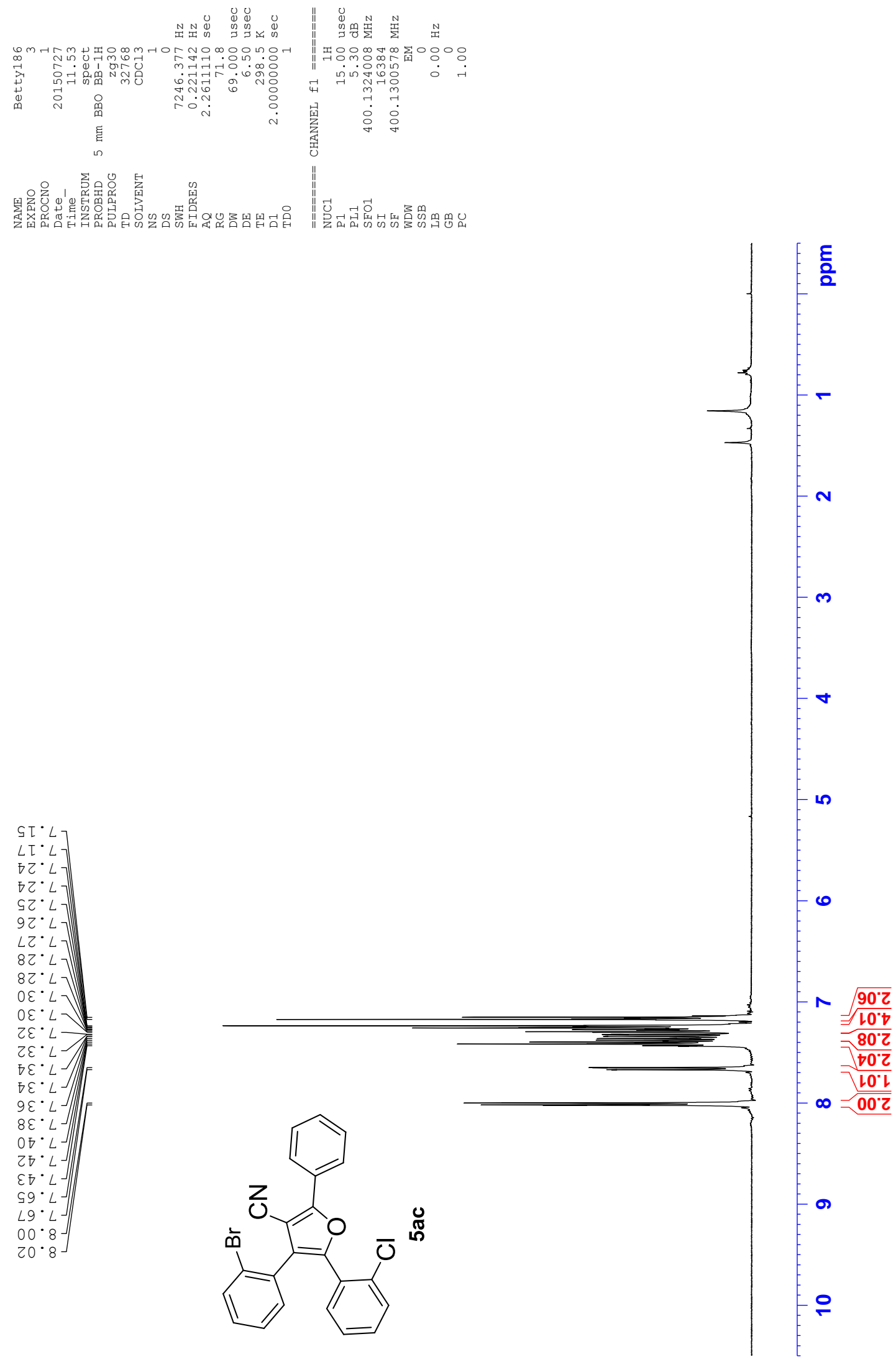

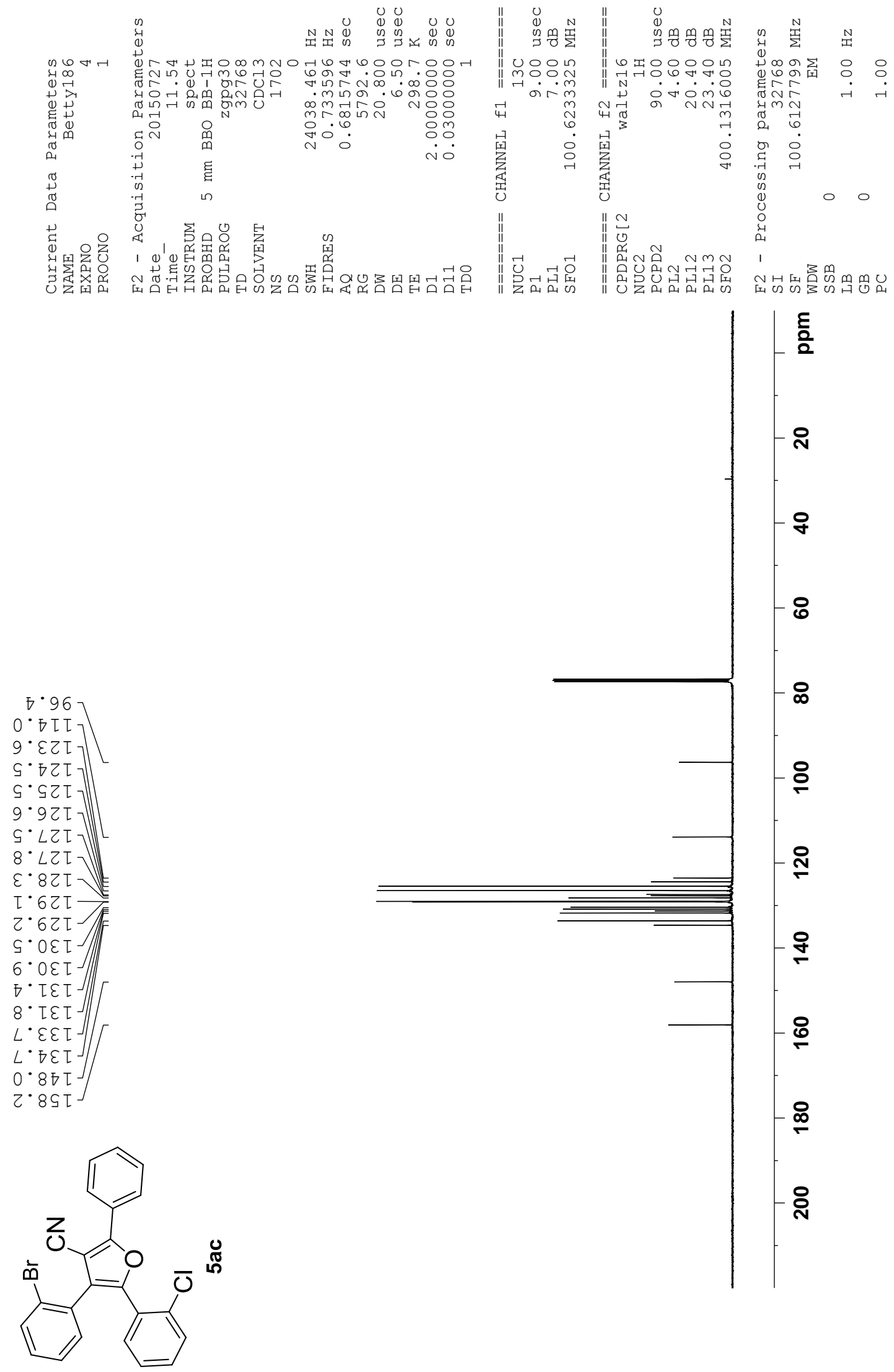

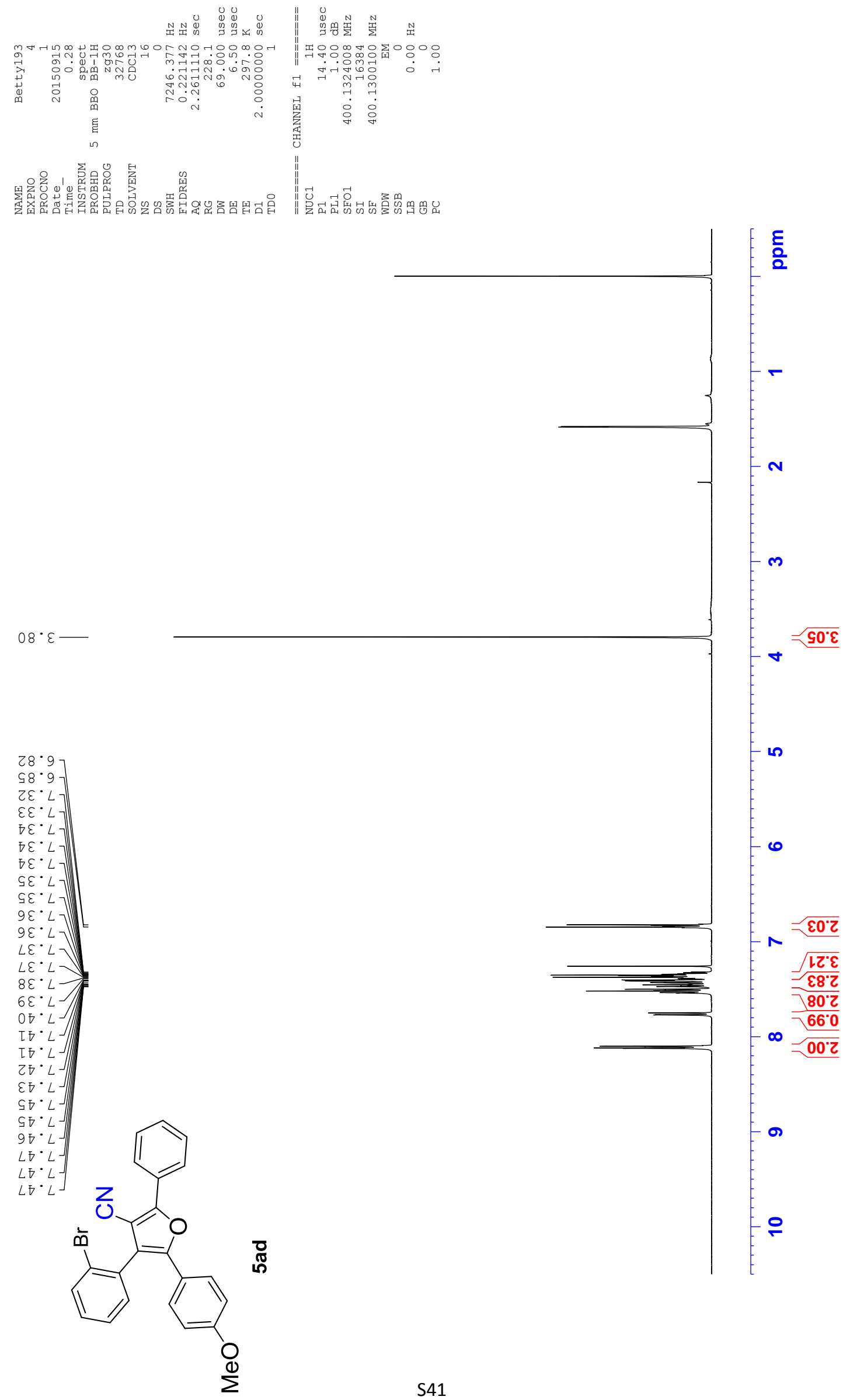

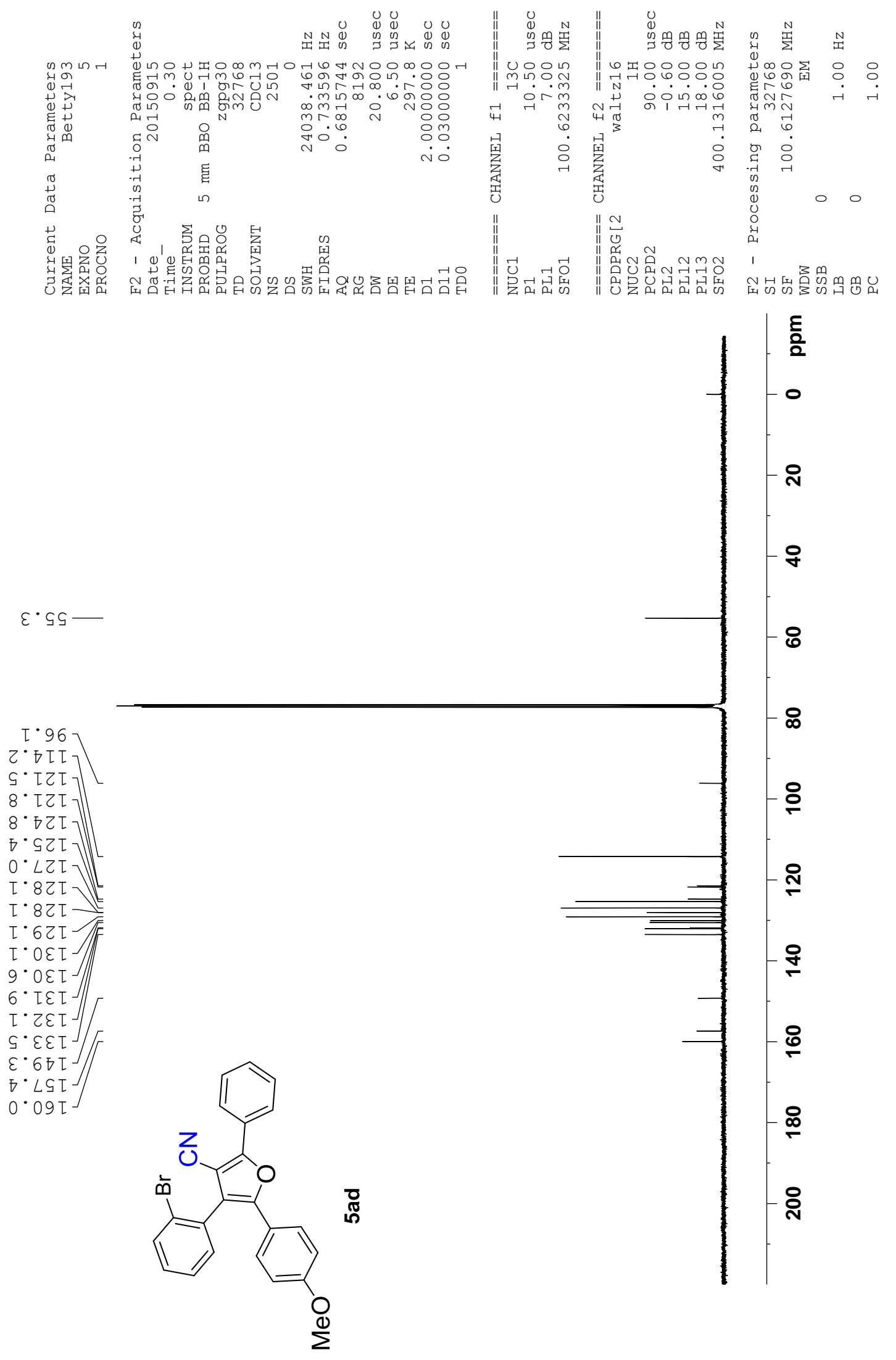

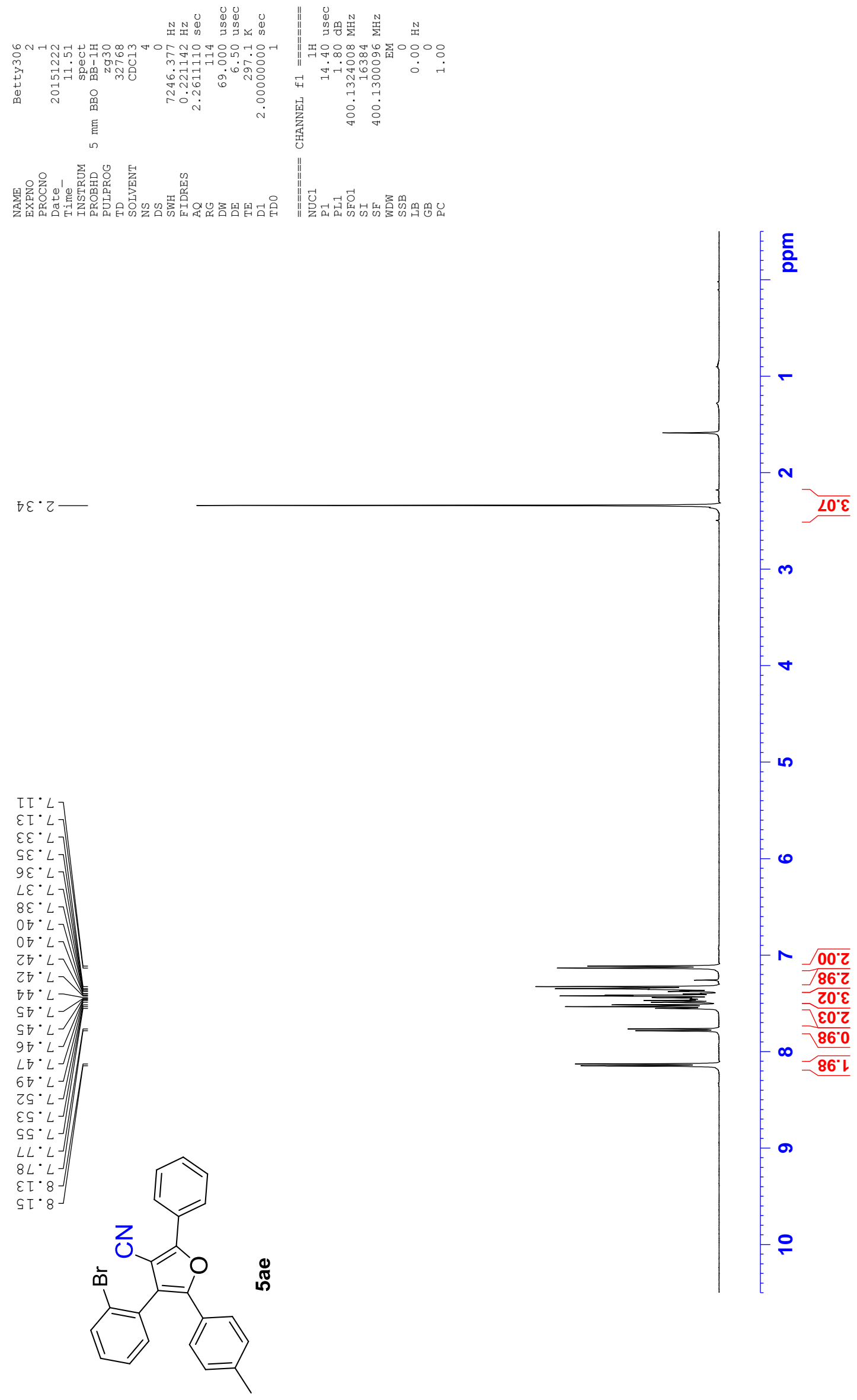

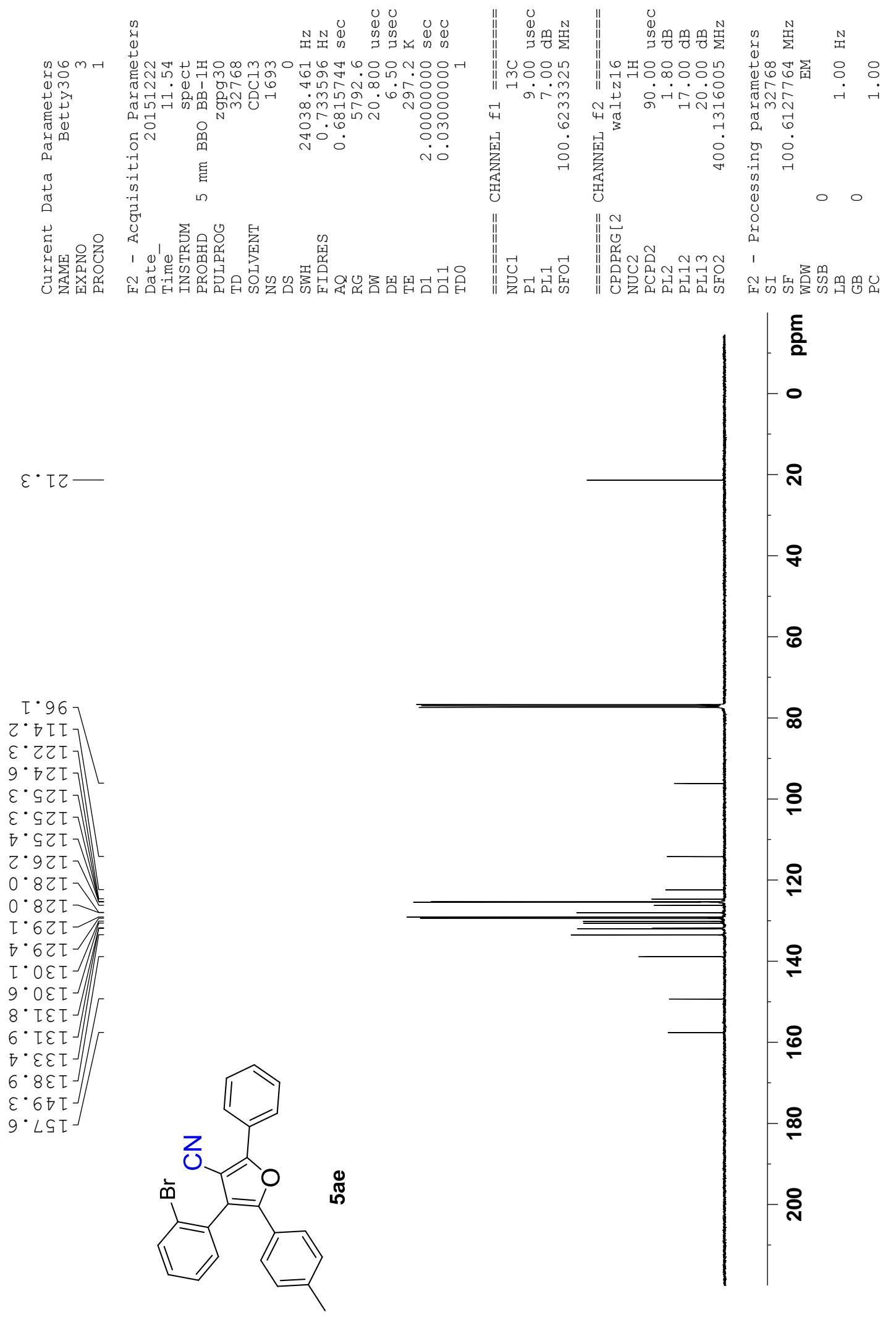

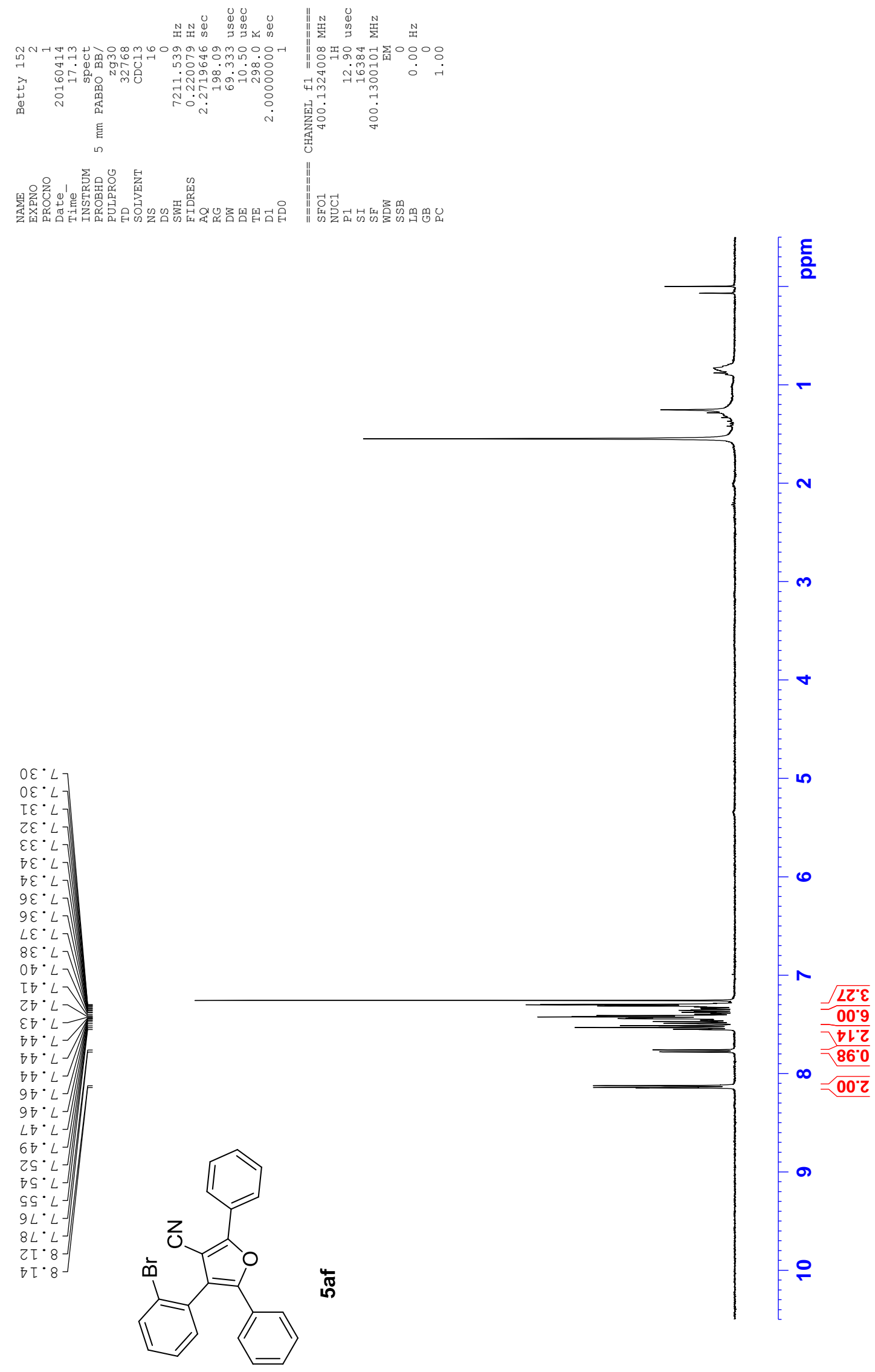

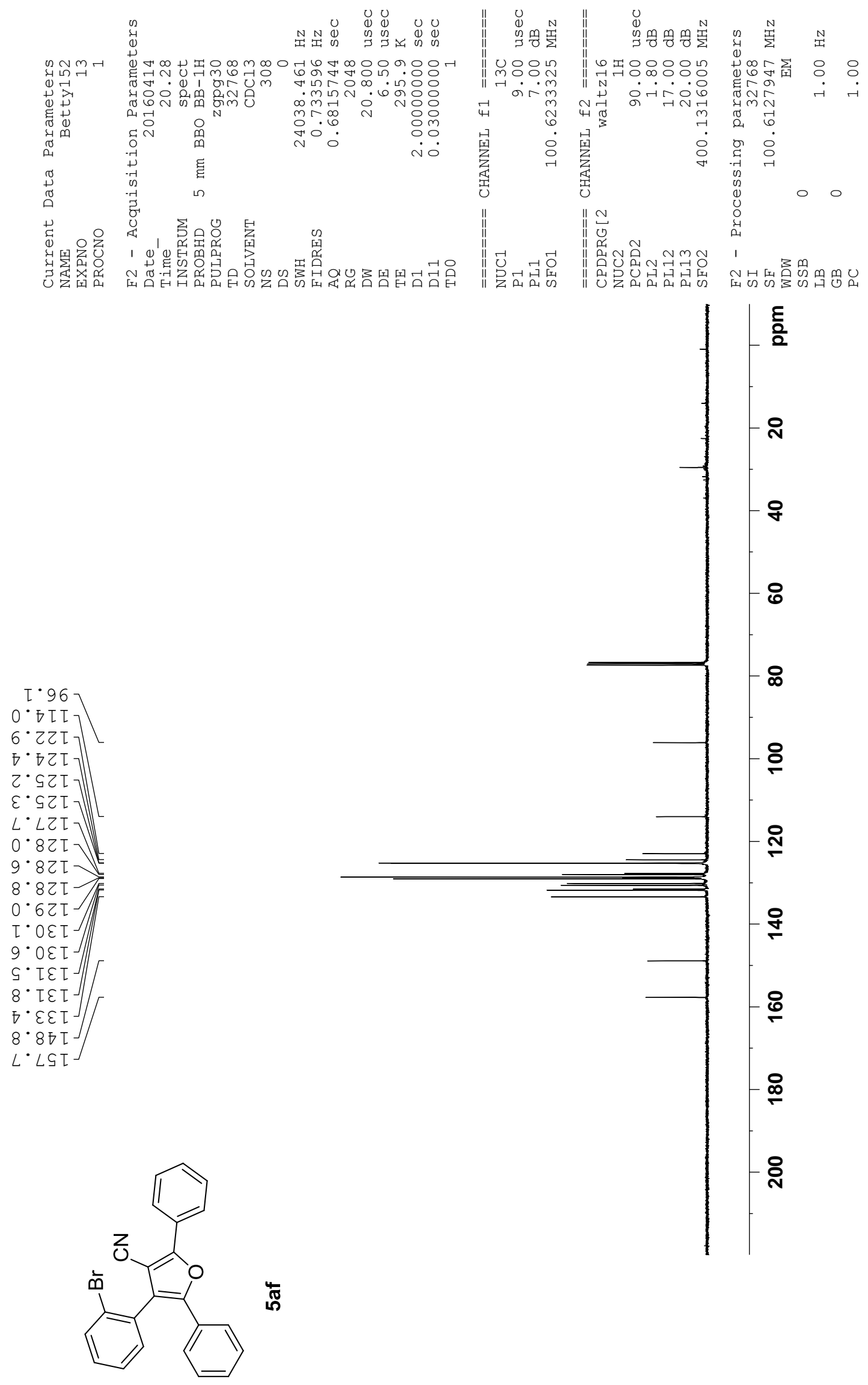

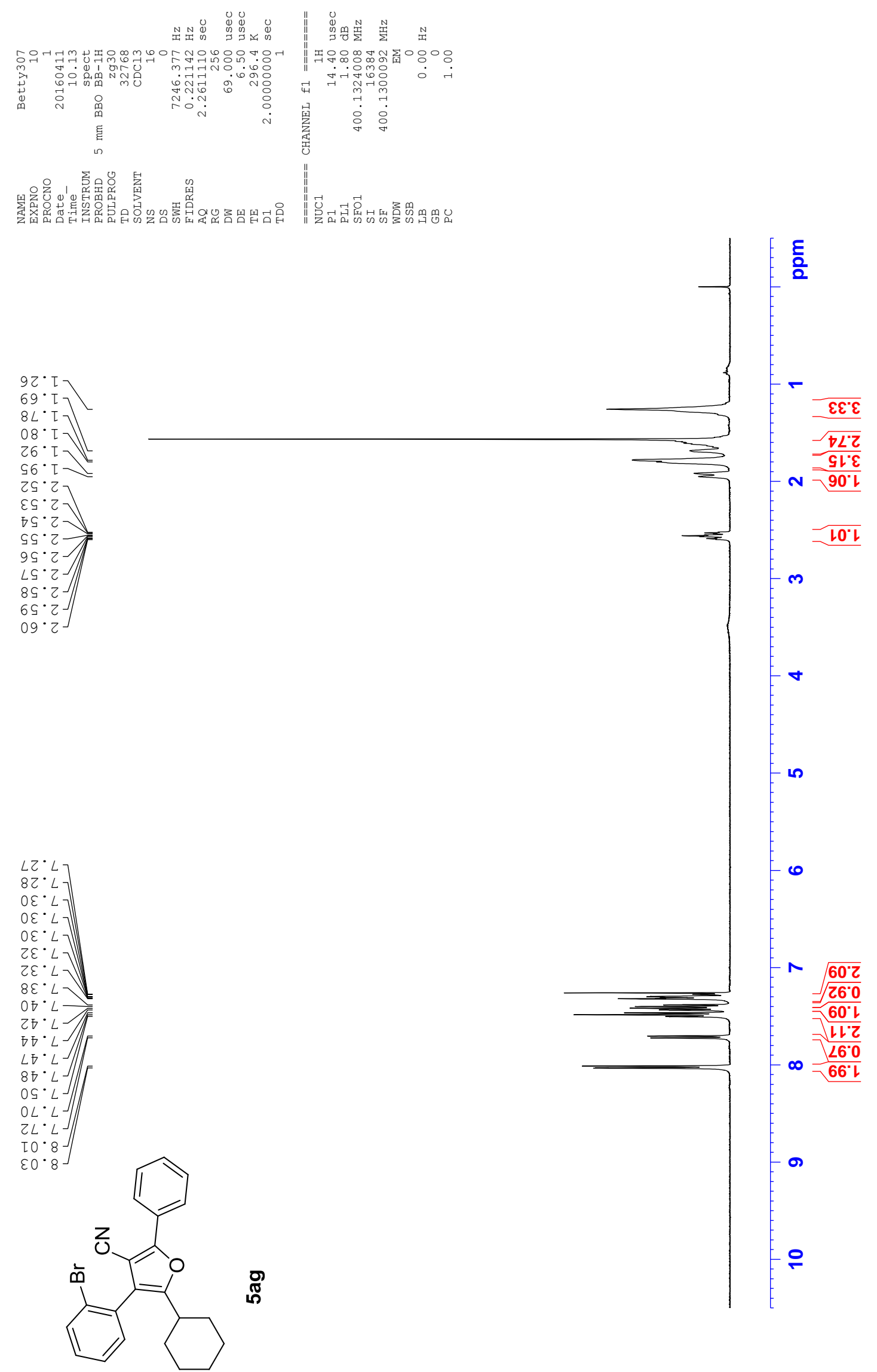

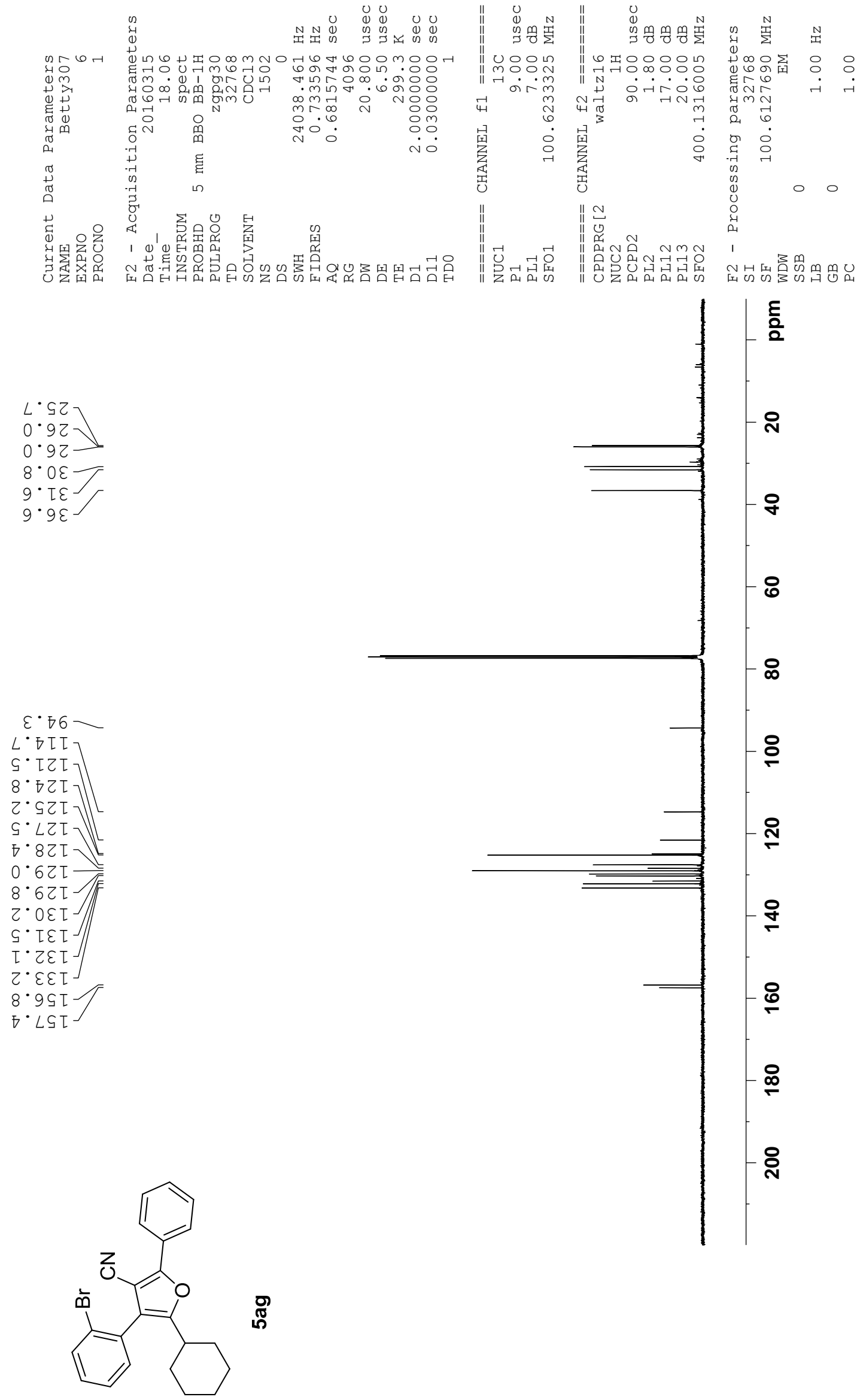


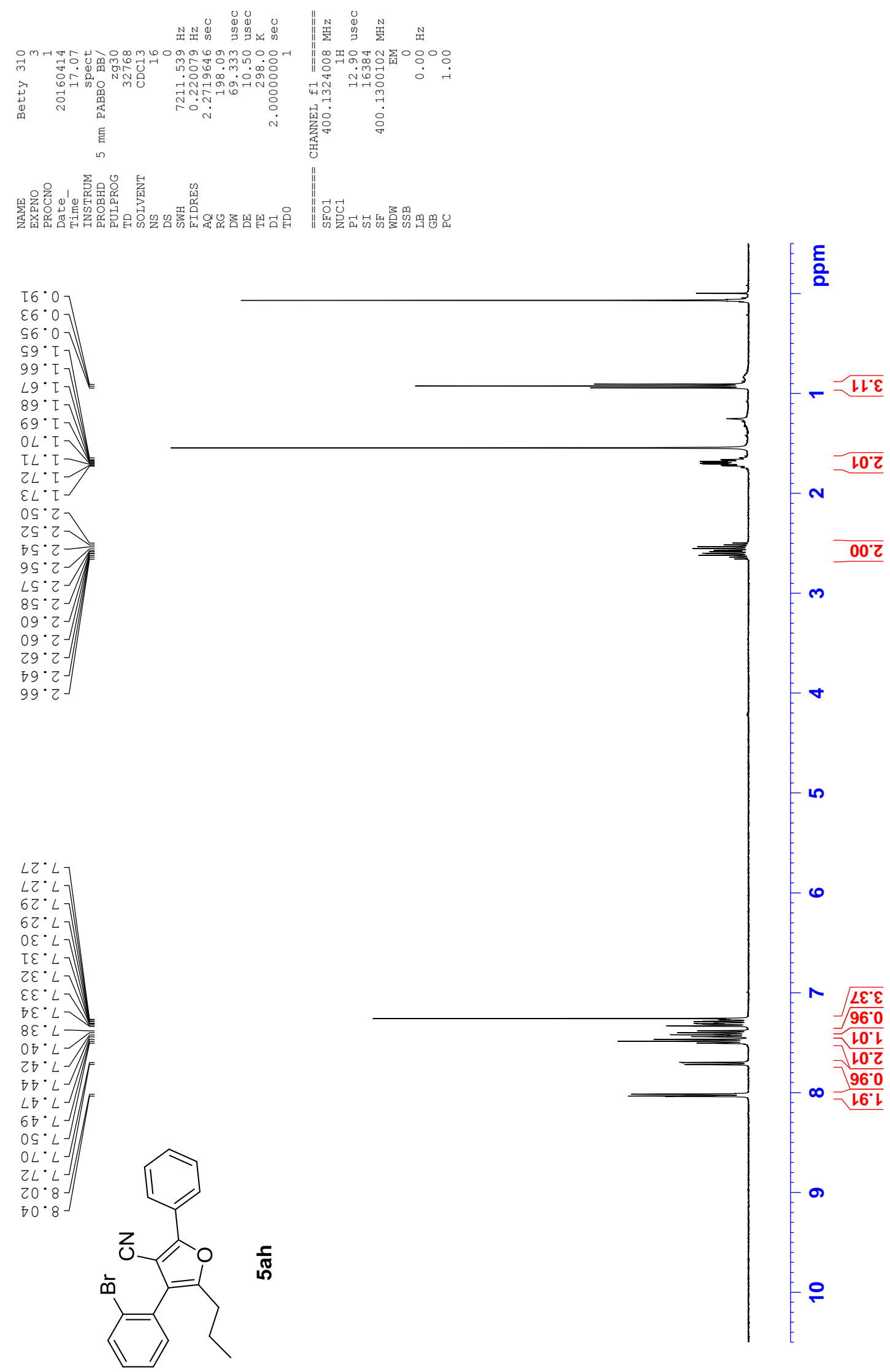



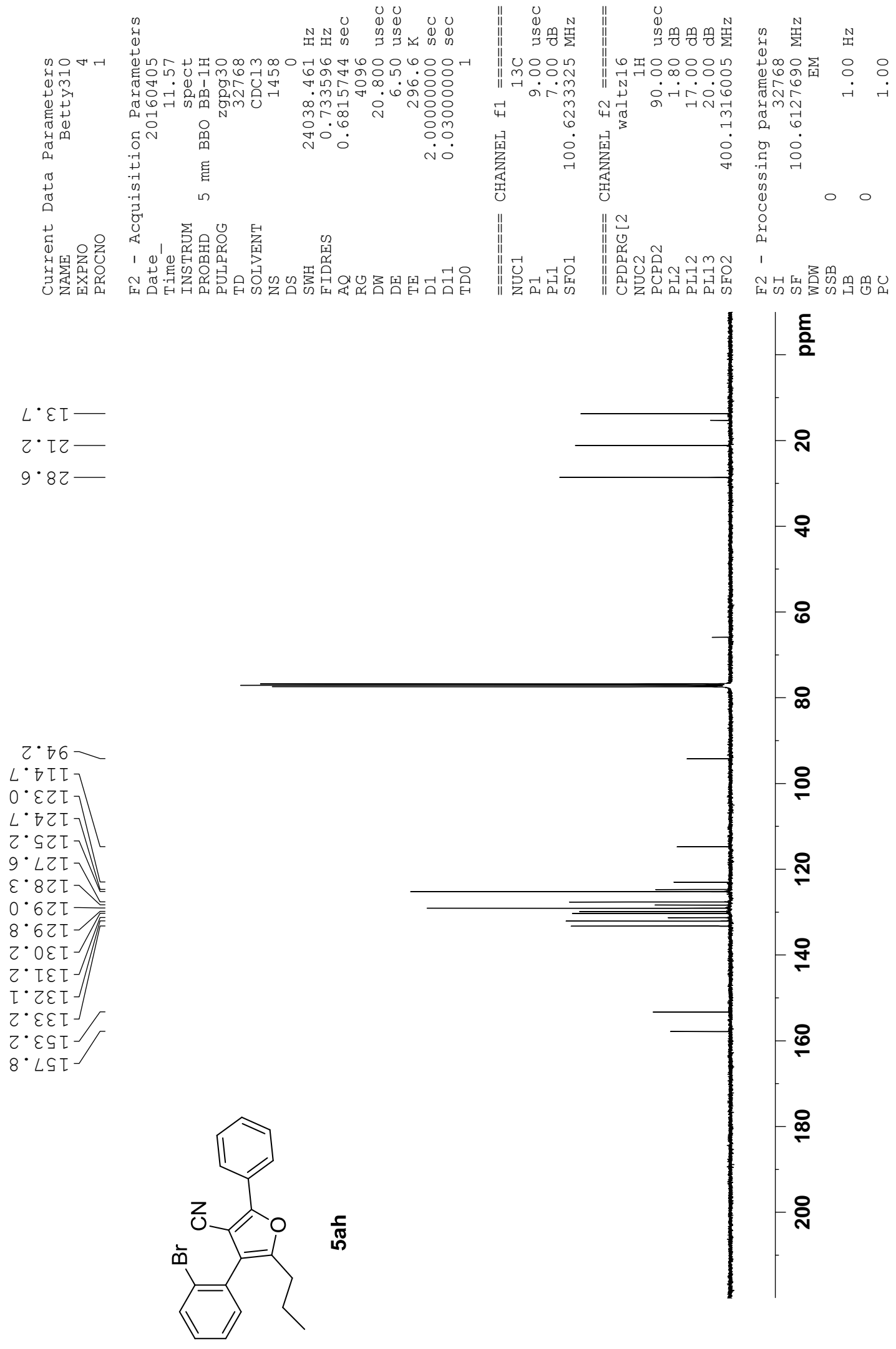

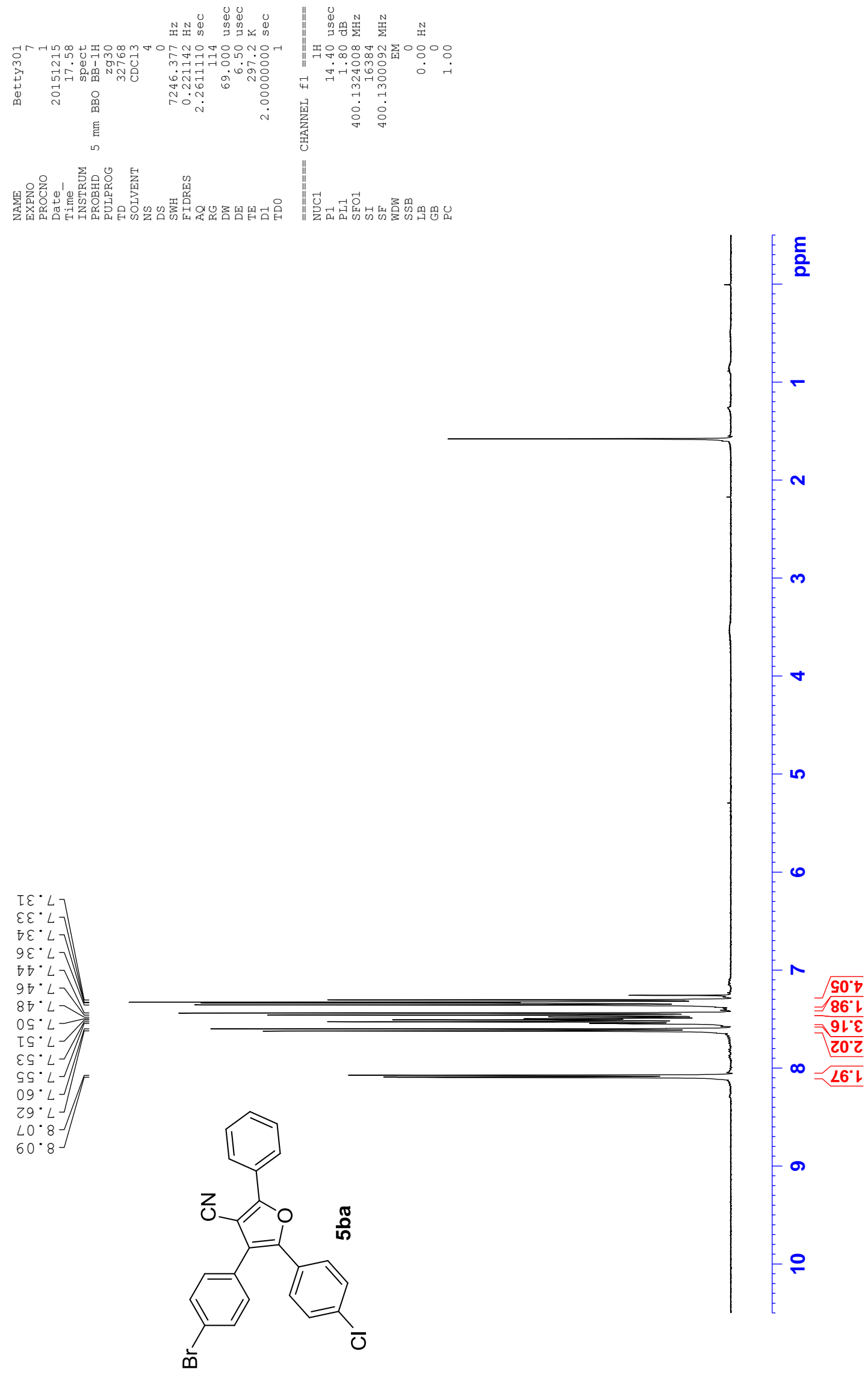


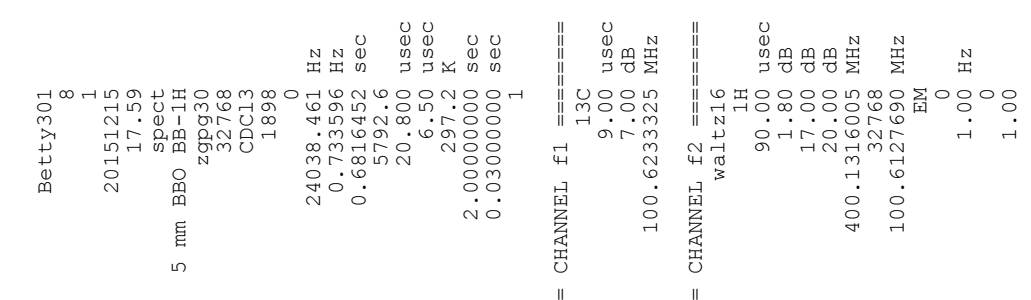

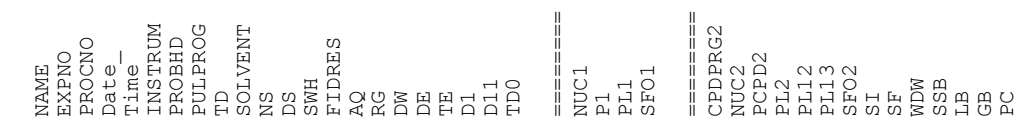
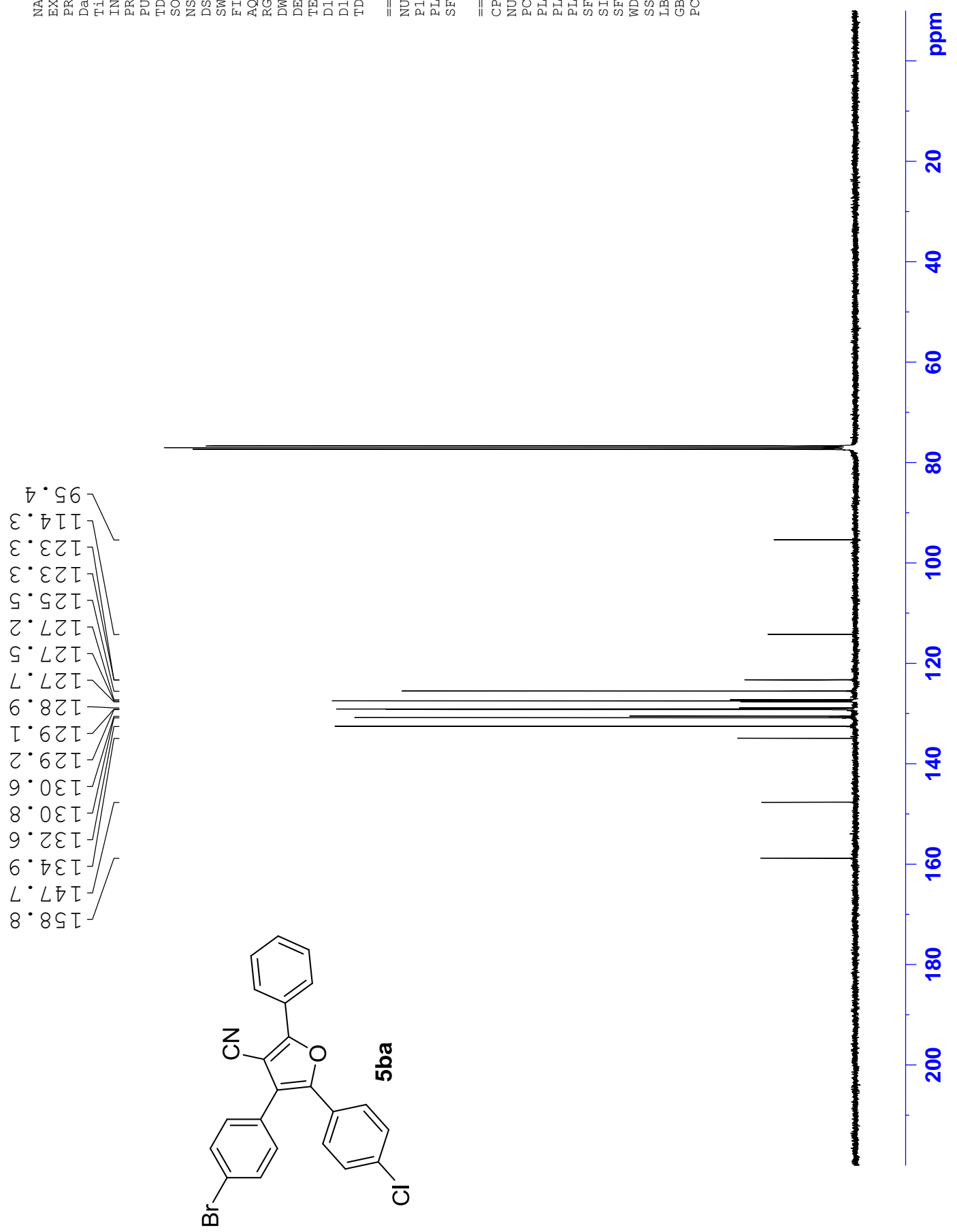

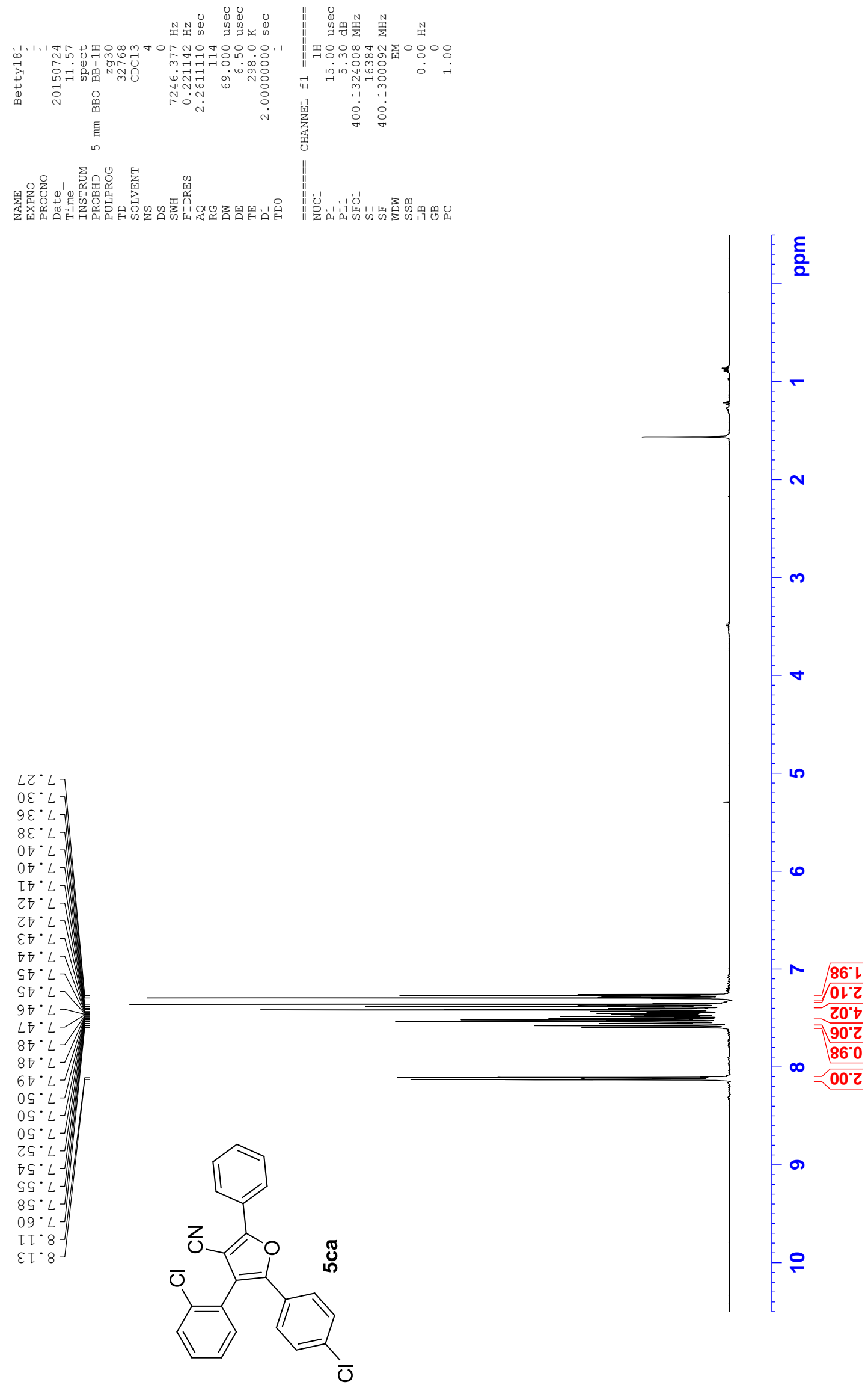


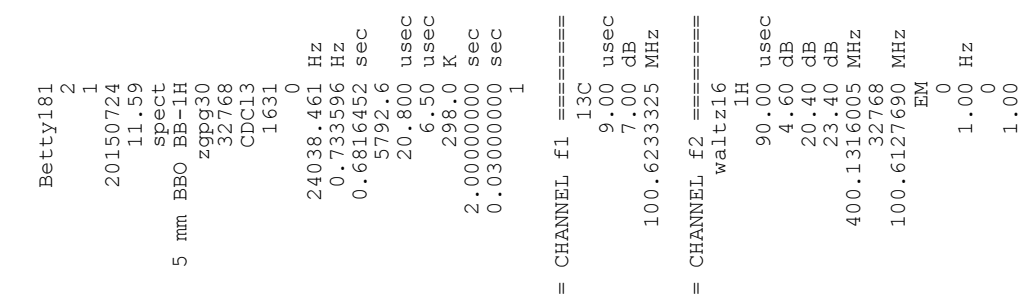

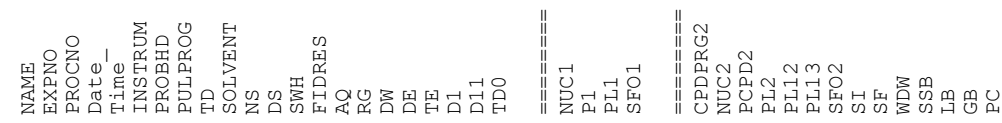

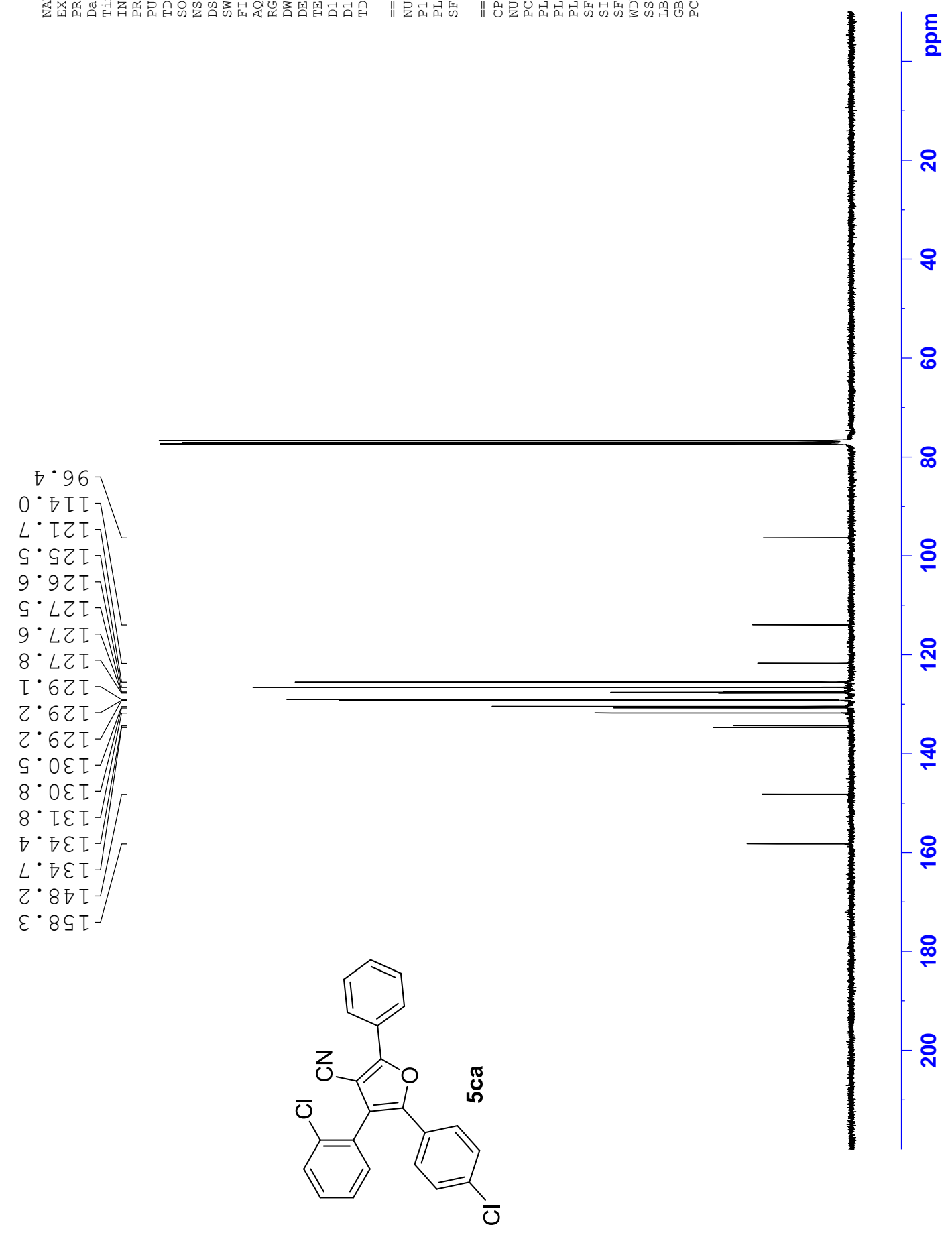



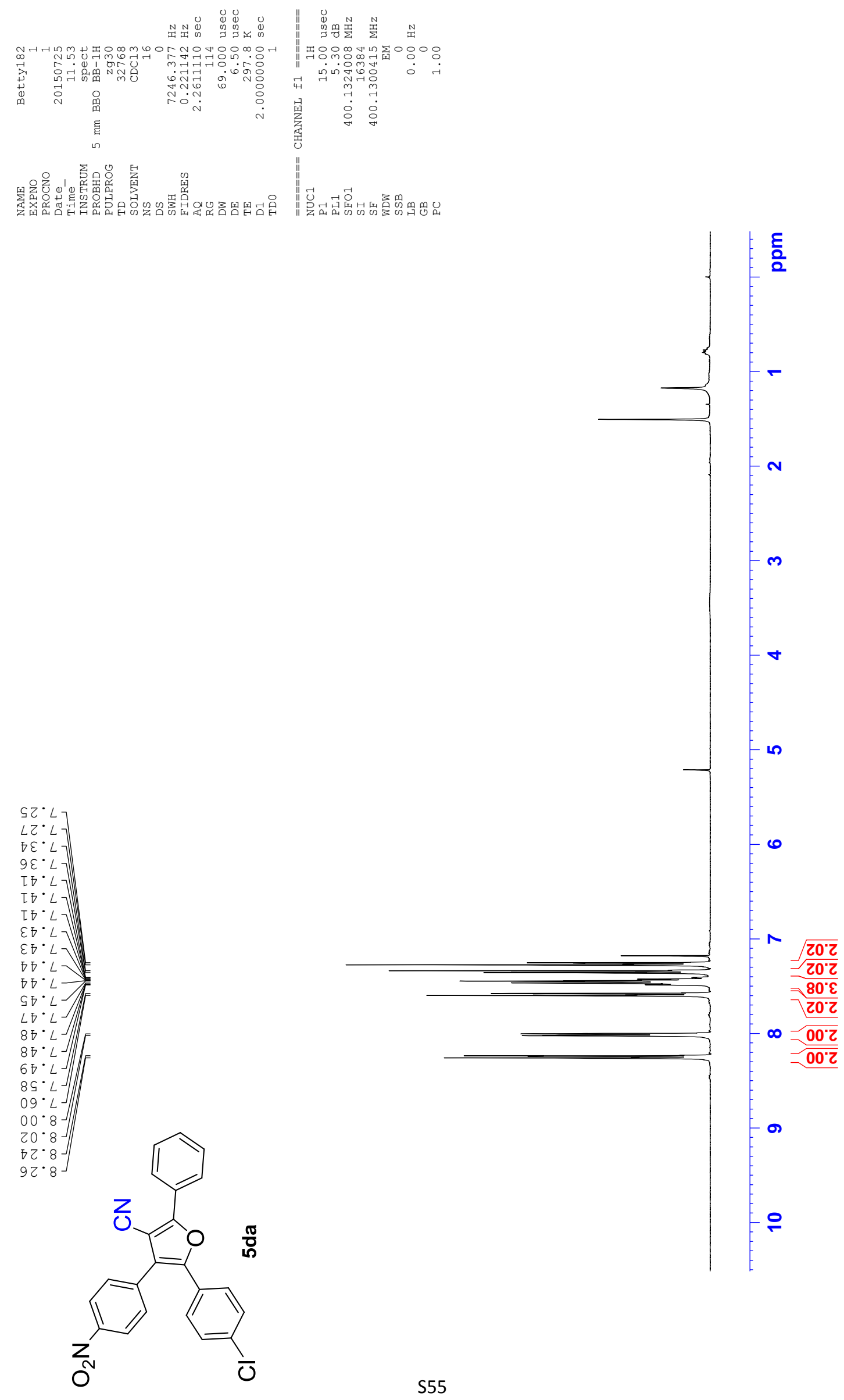

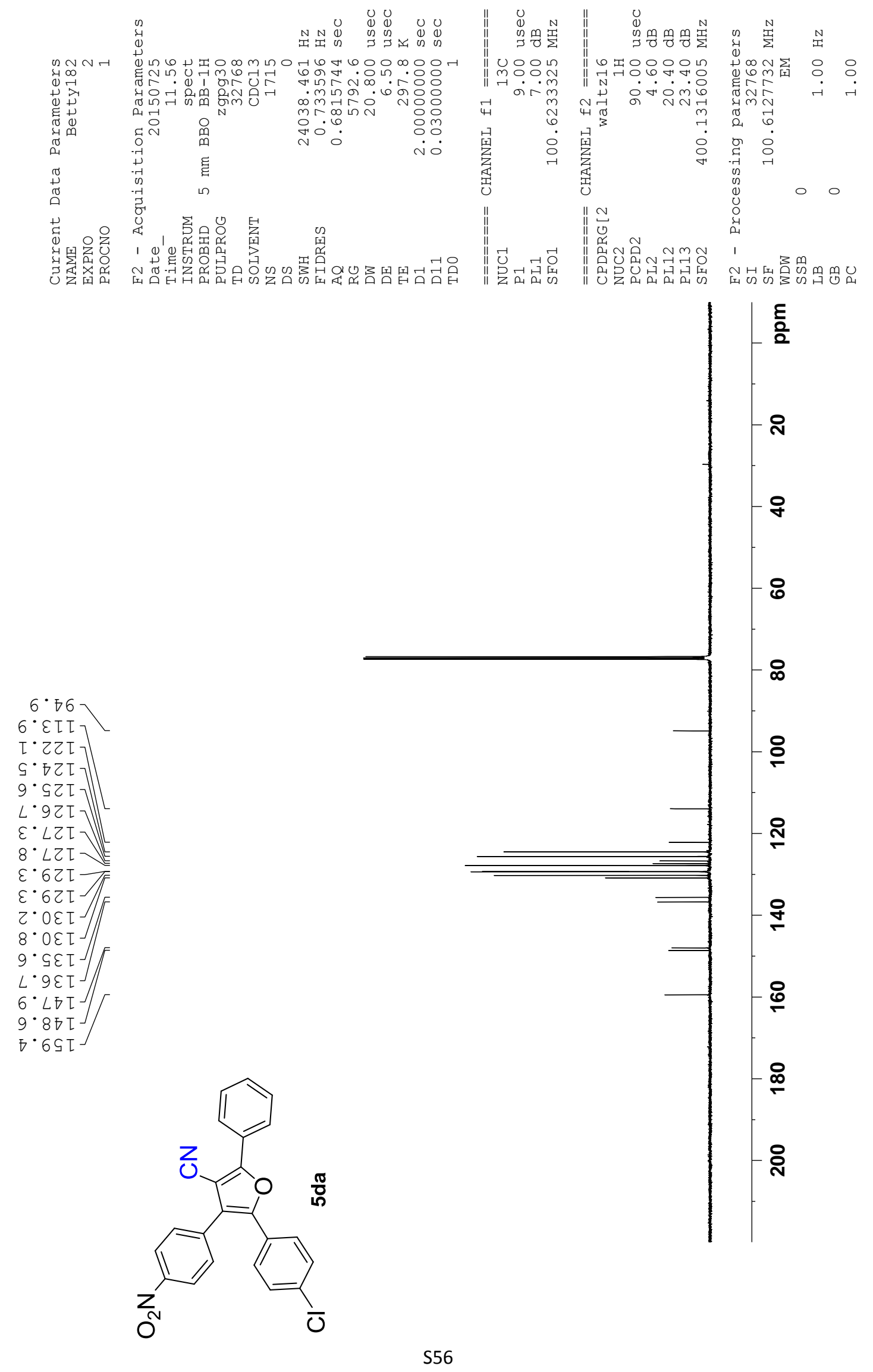

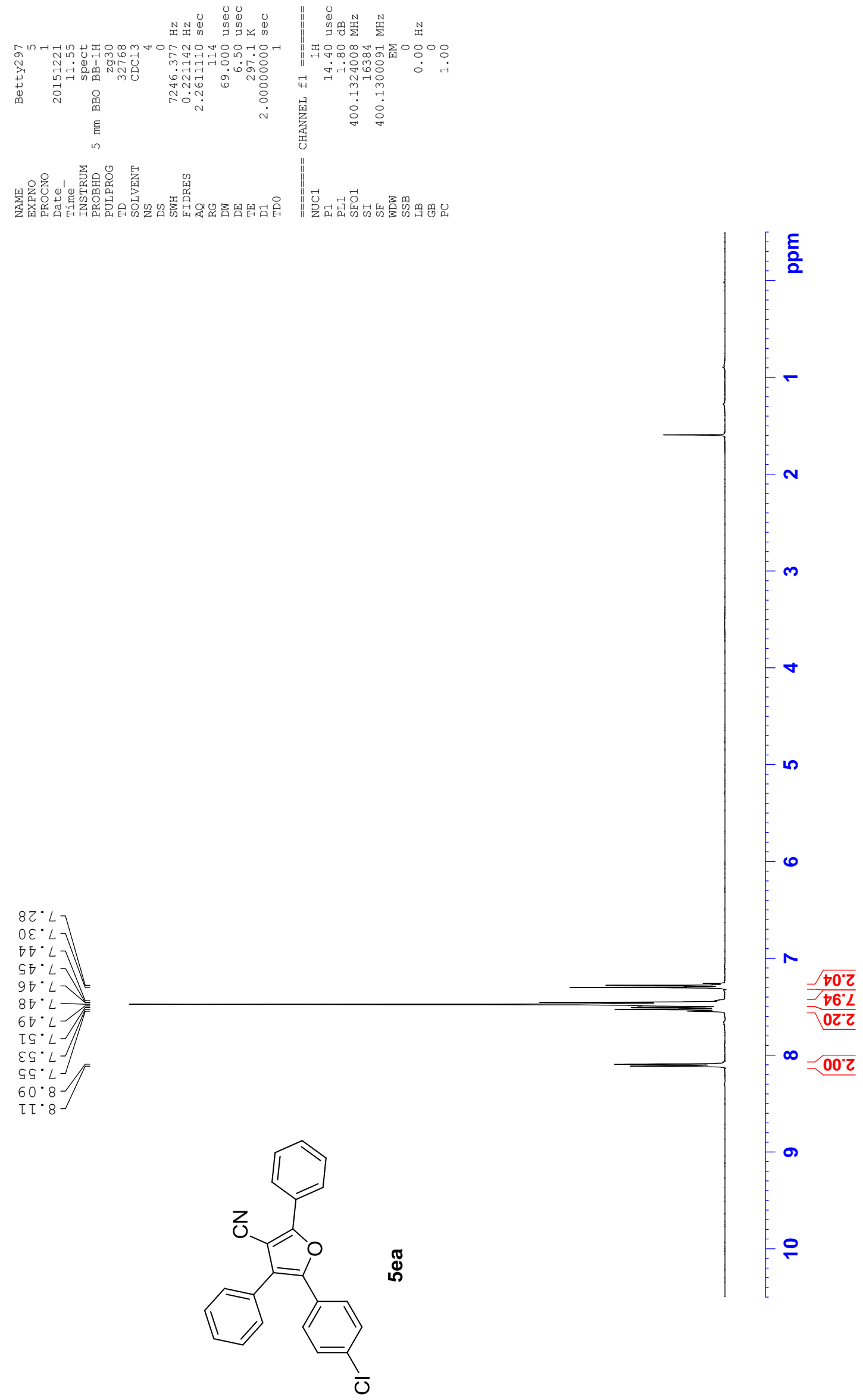

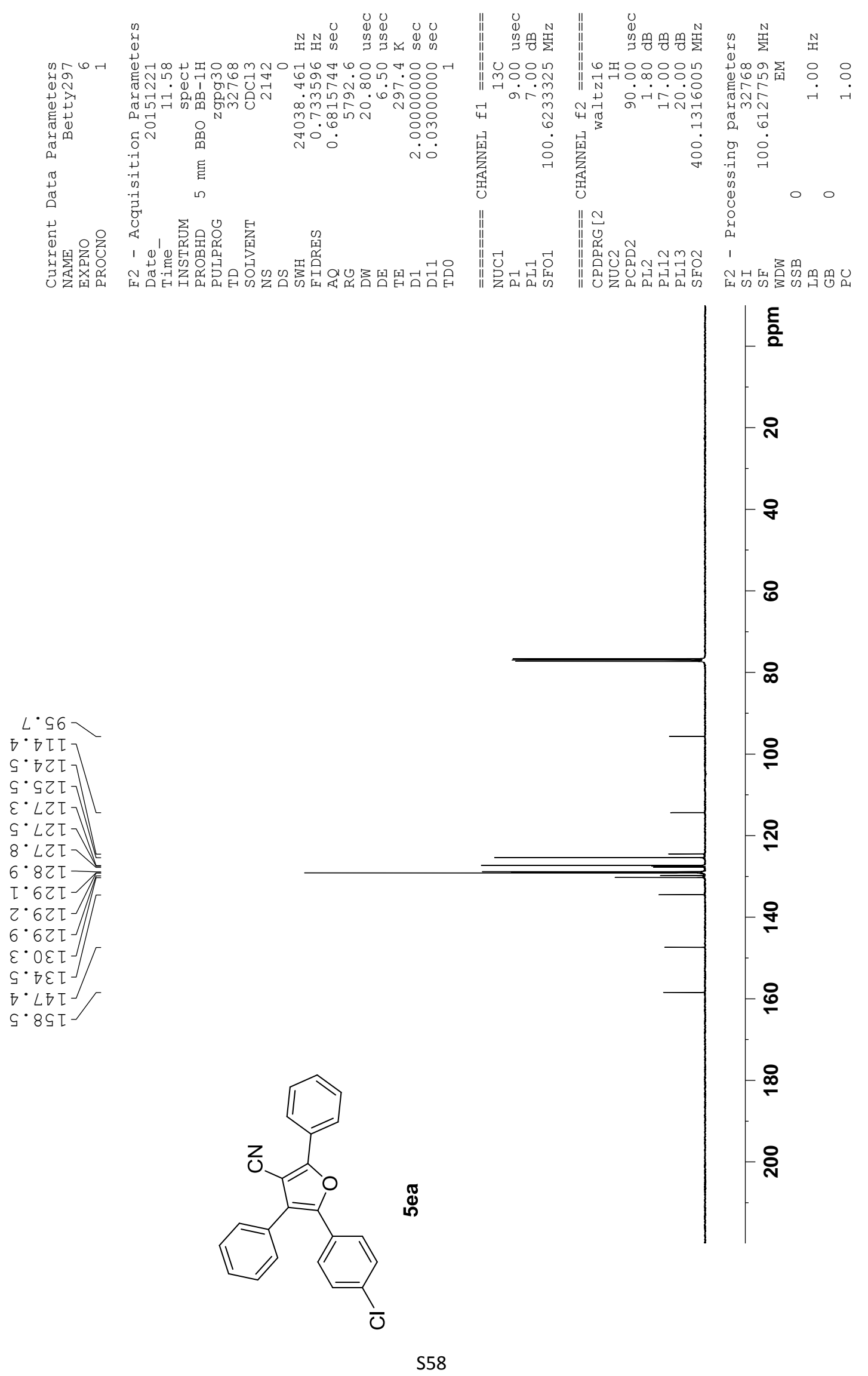

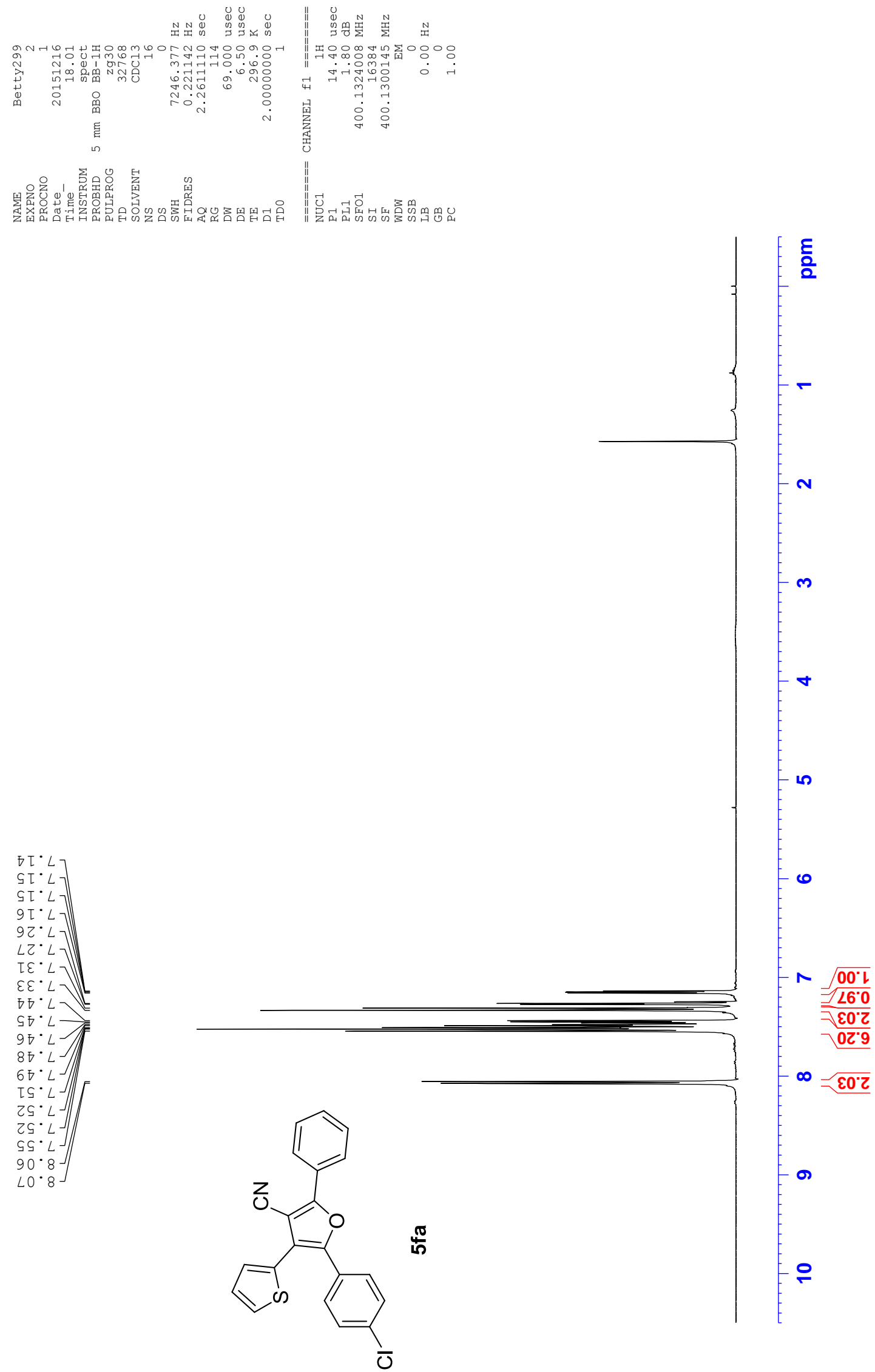

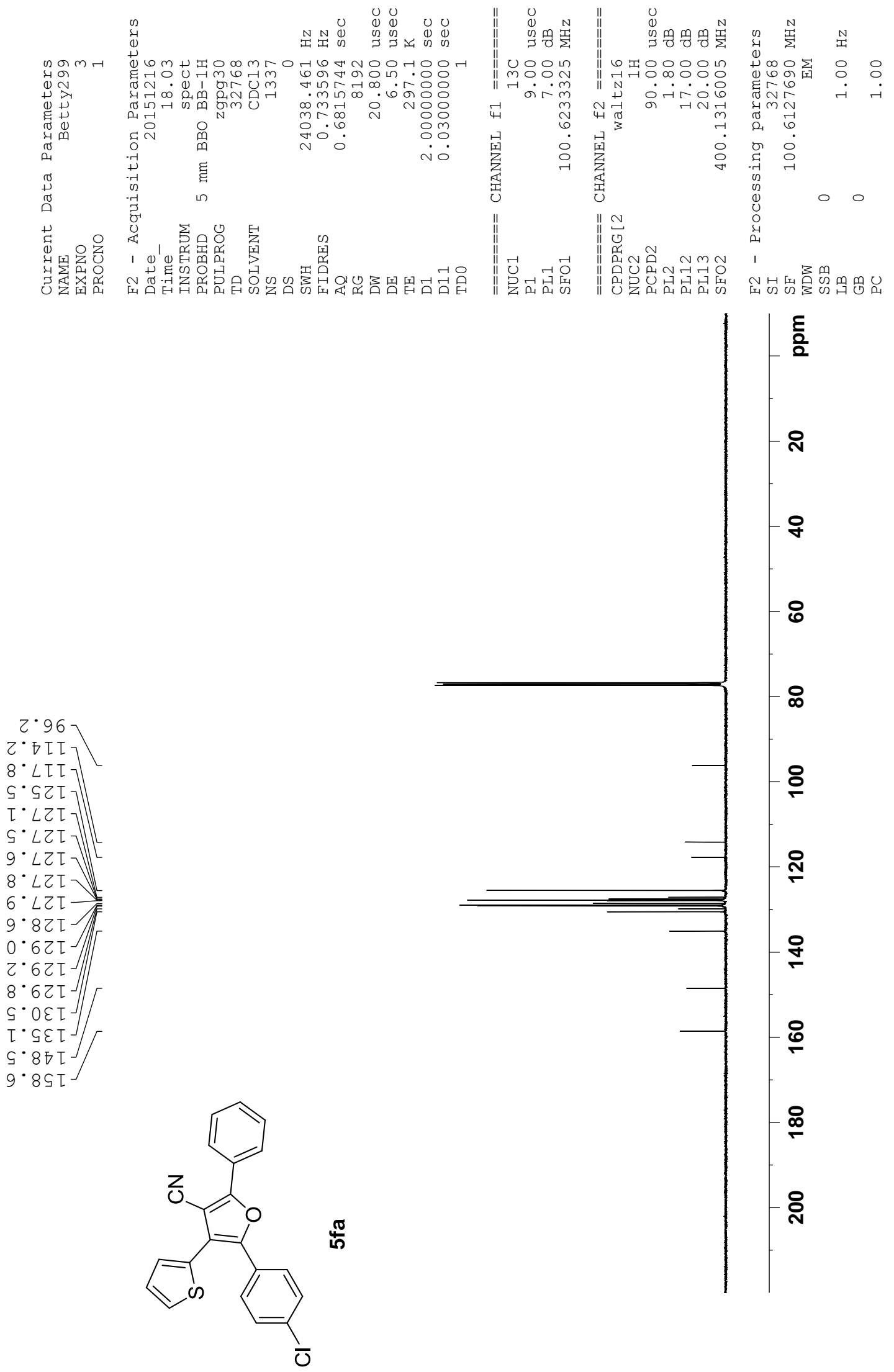

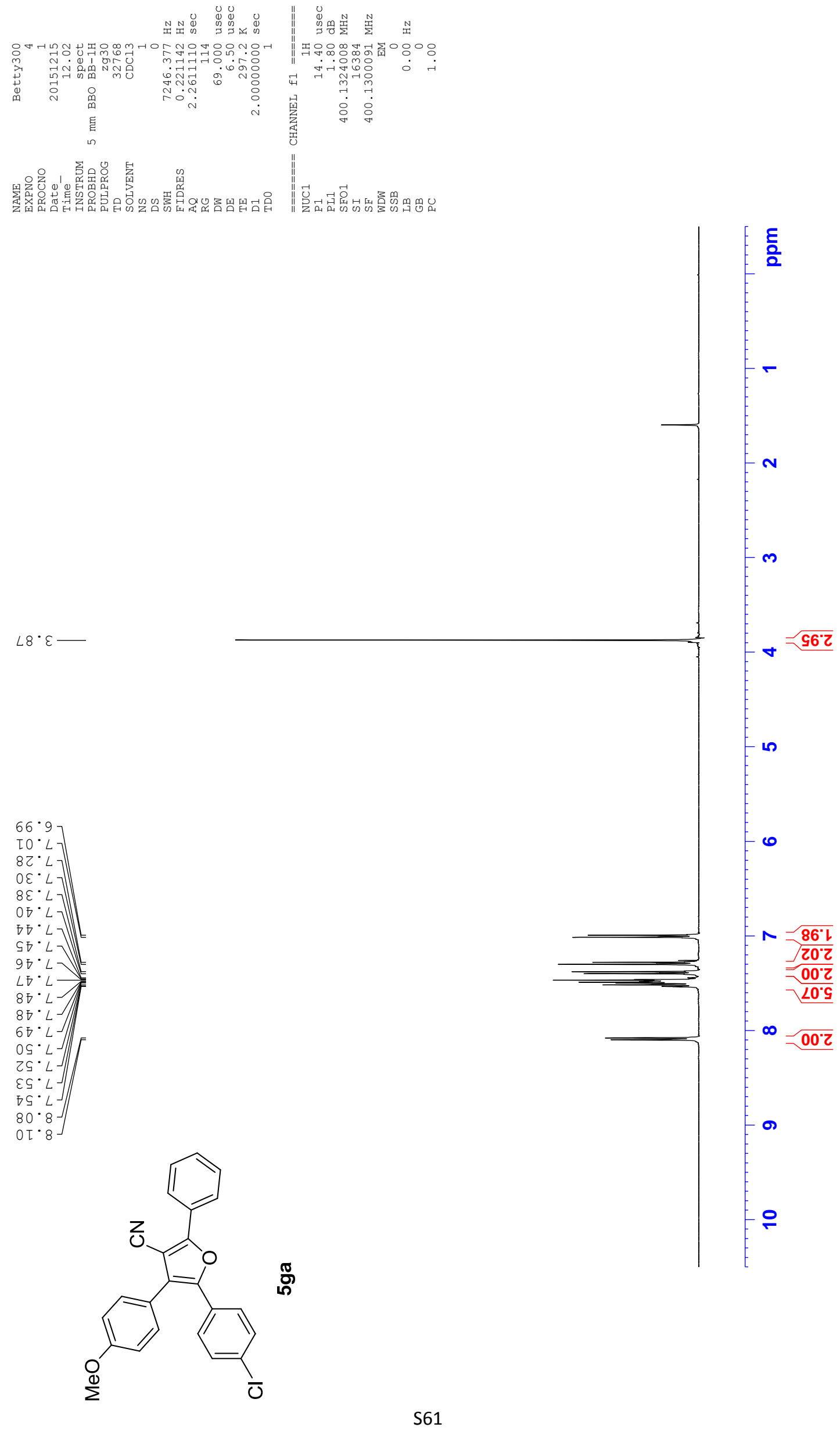

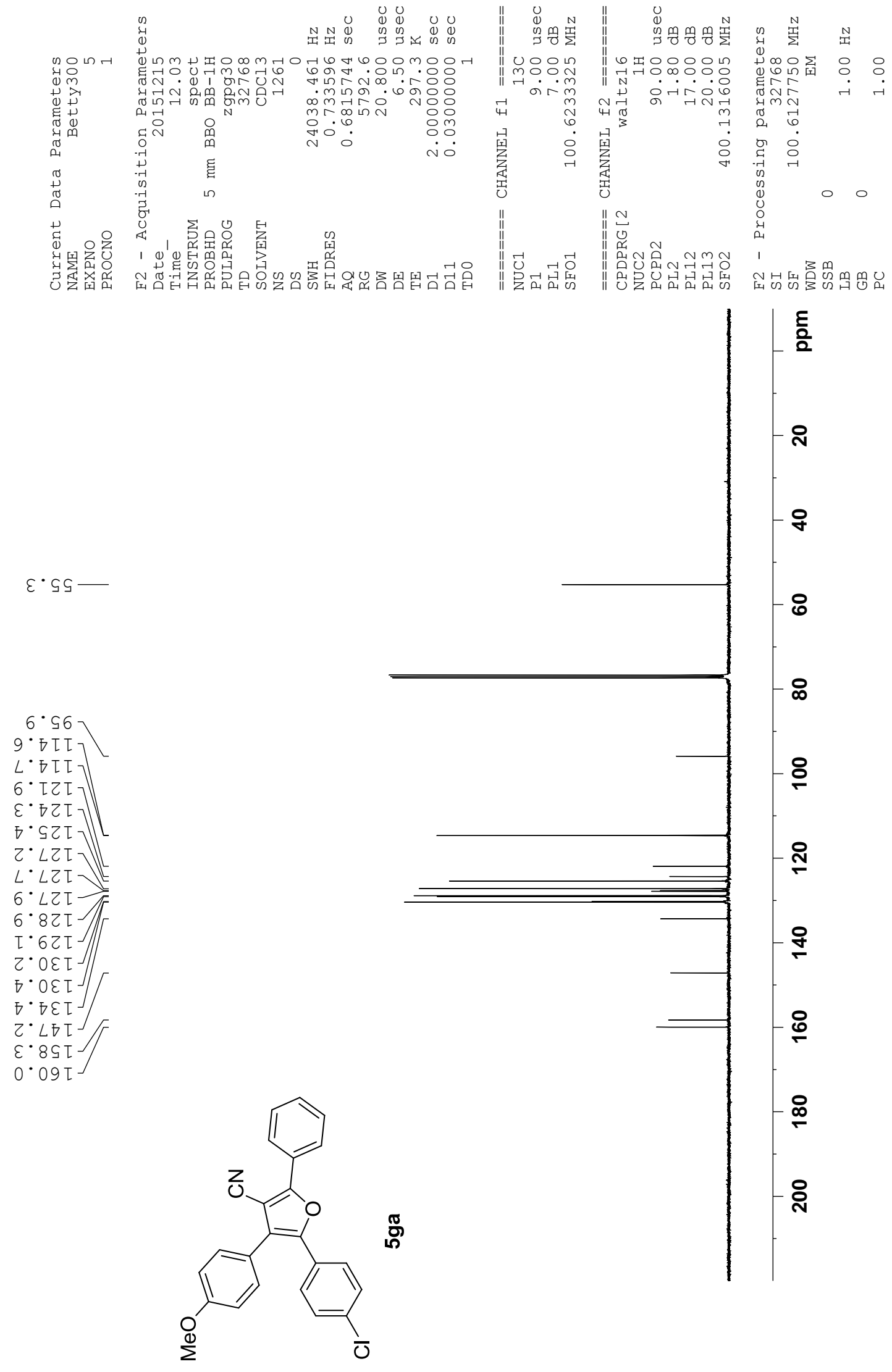


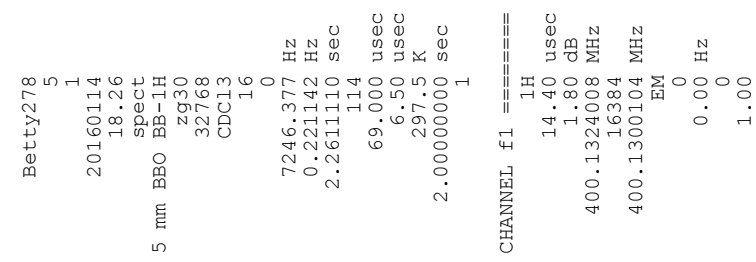

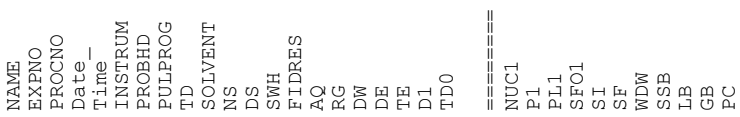

$46 \cdot 0$

$66^{\circ} 0$

[0 ${ }^{\circ}$
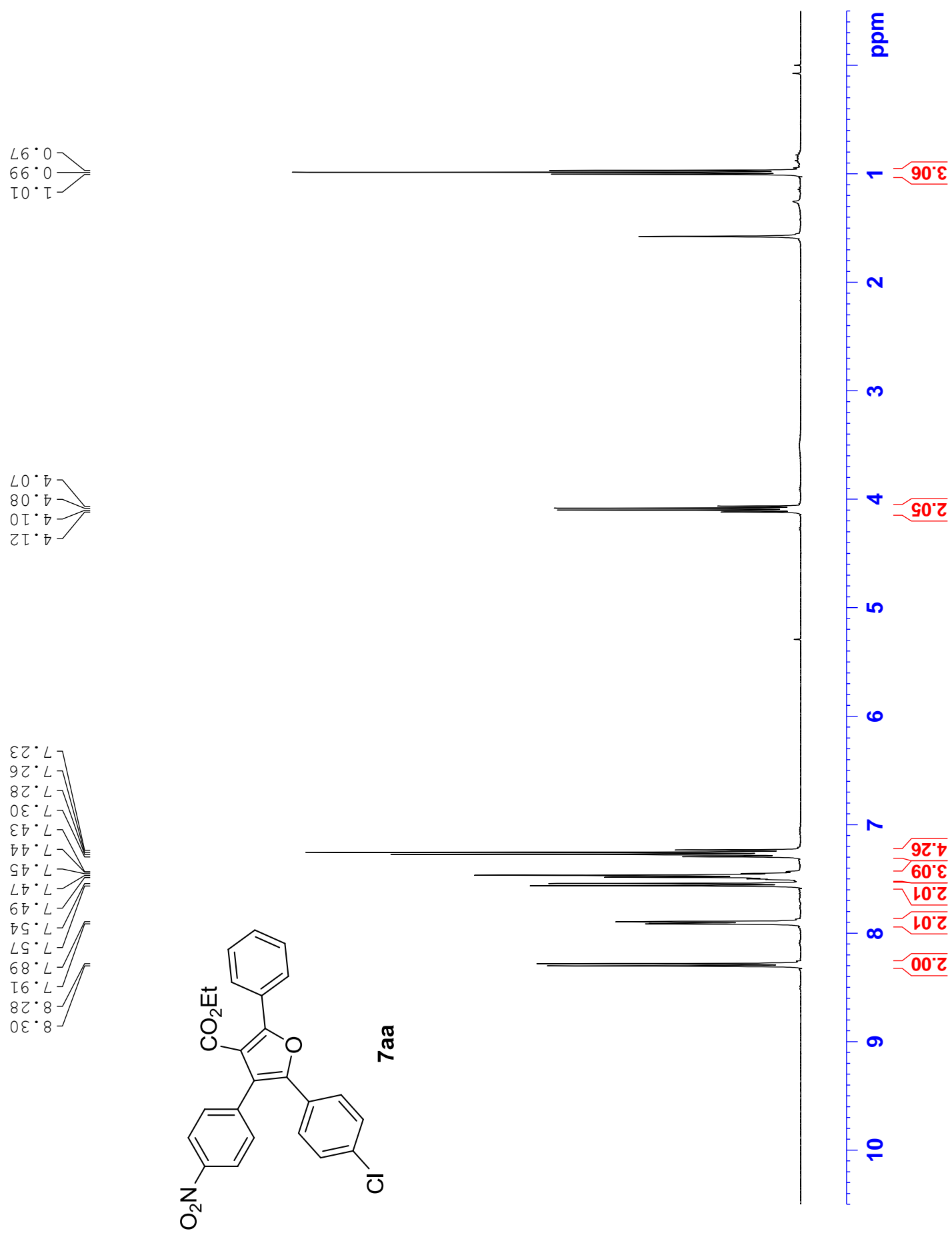


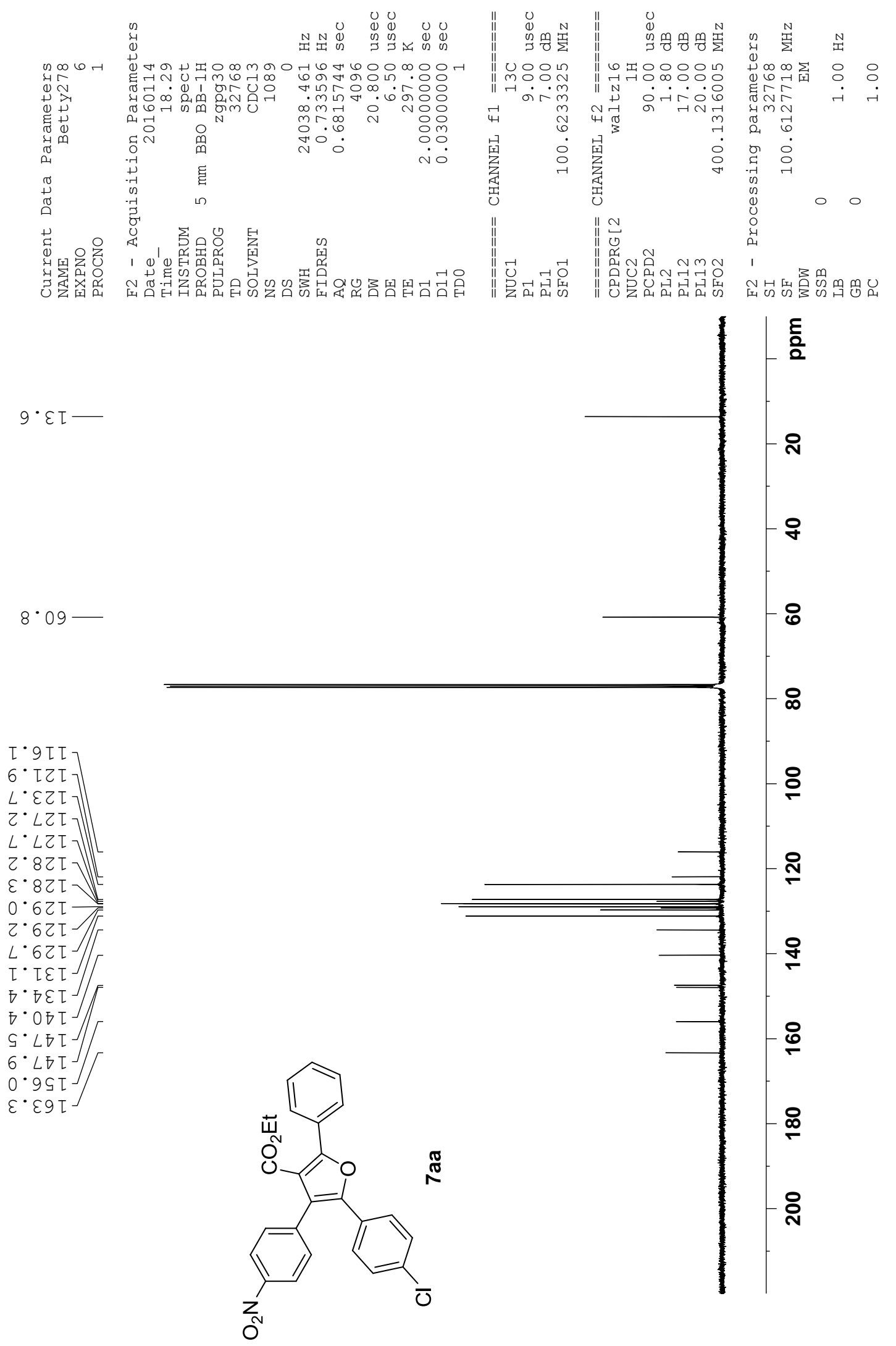




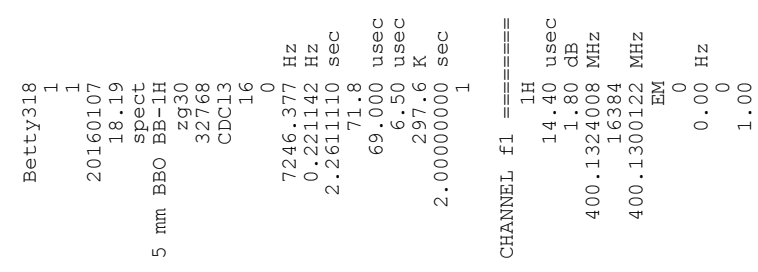

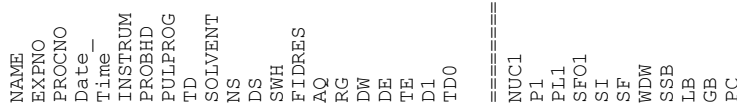

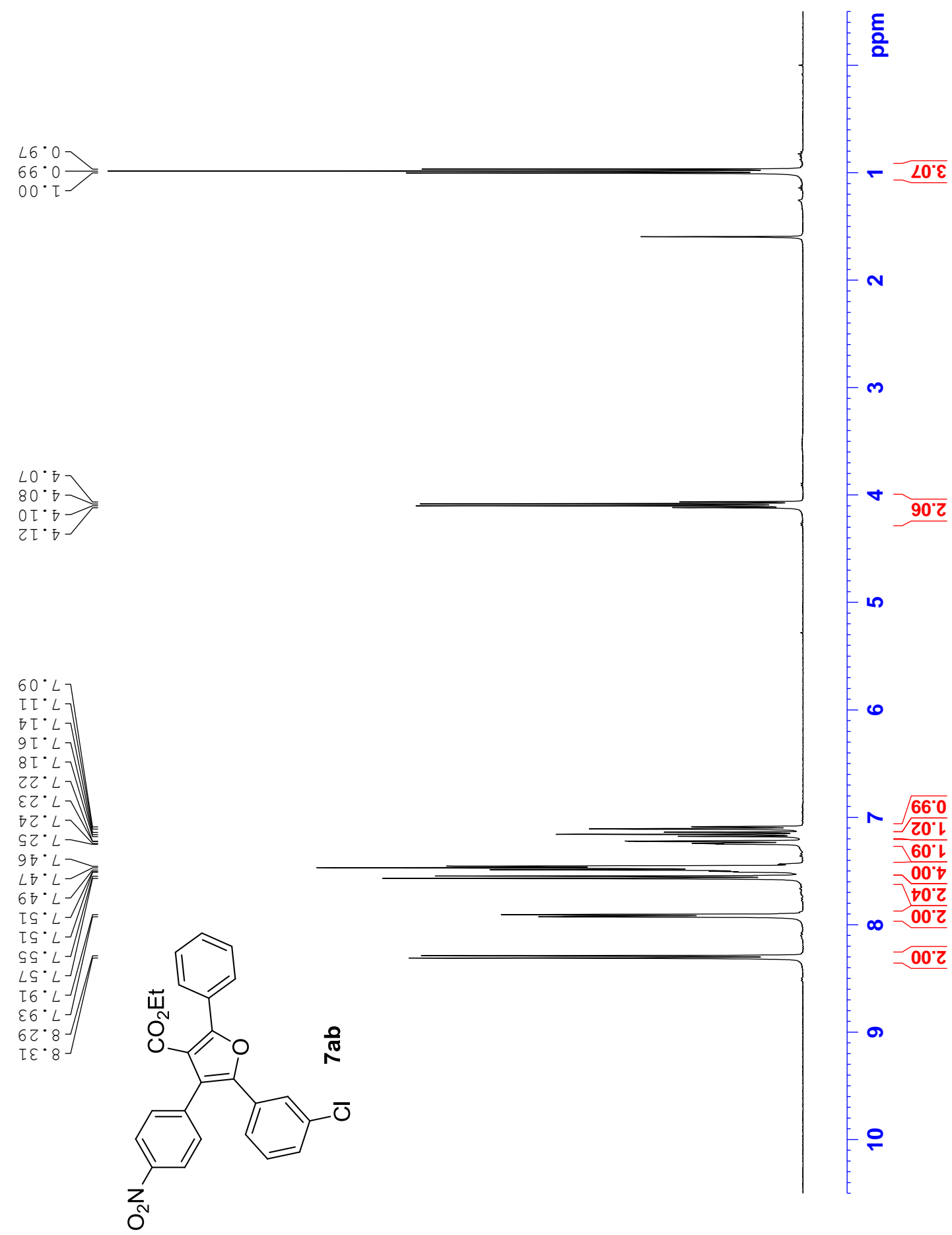



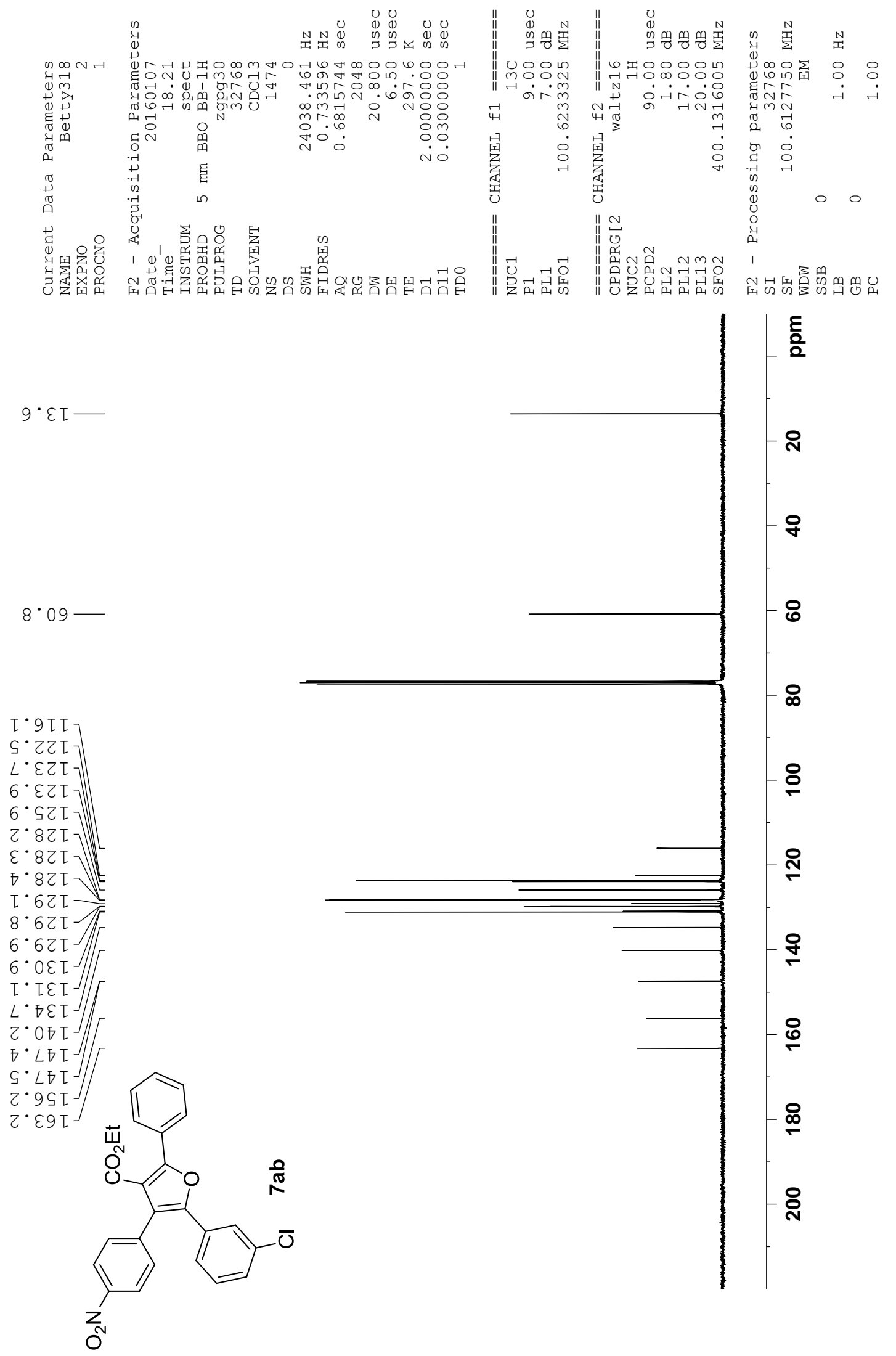


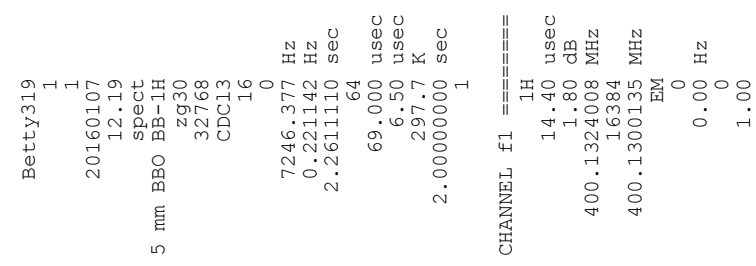

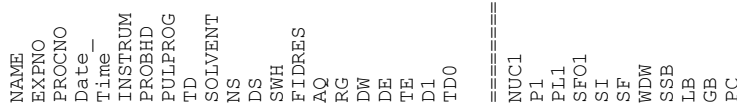
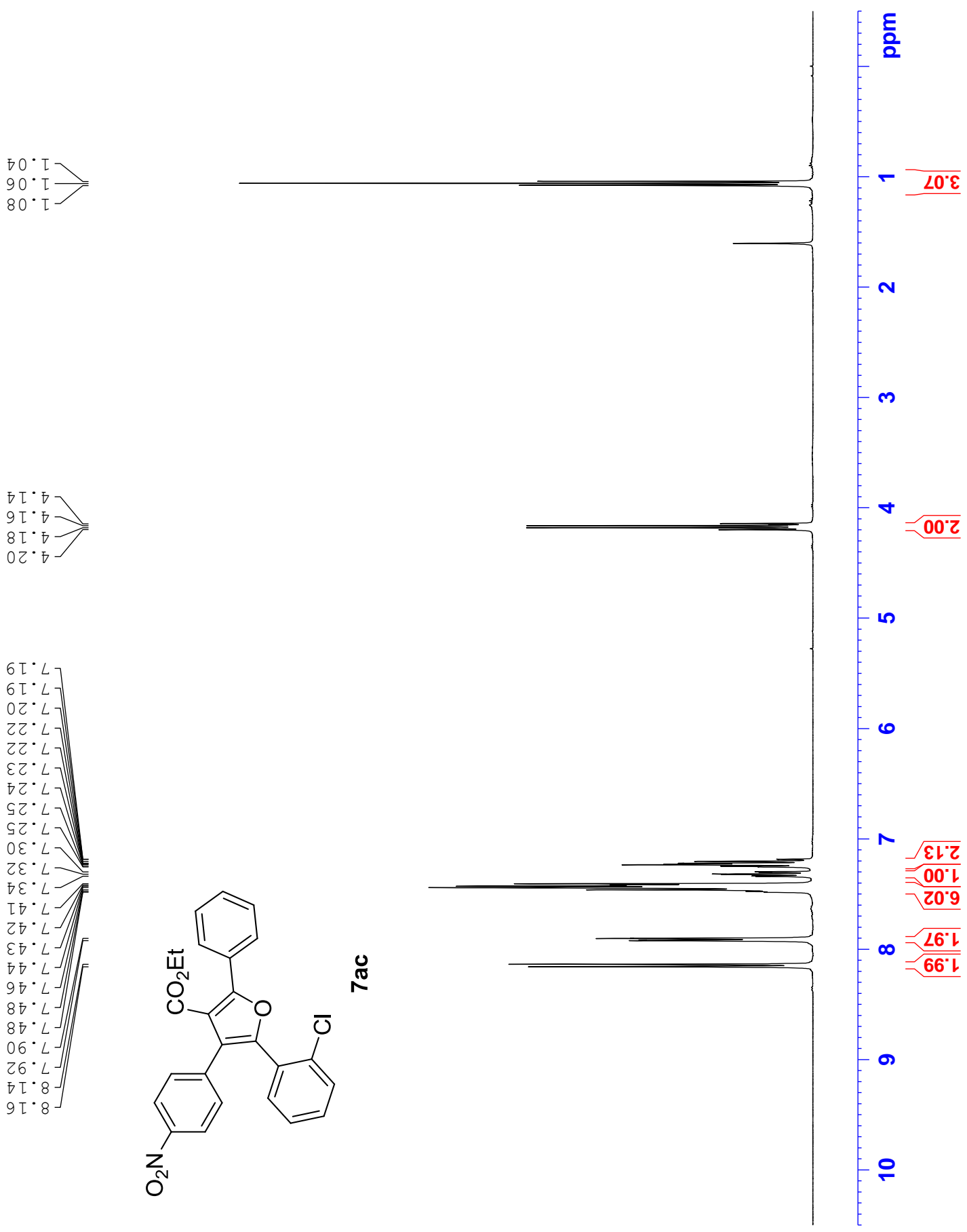


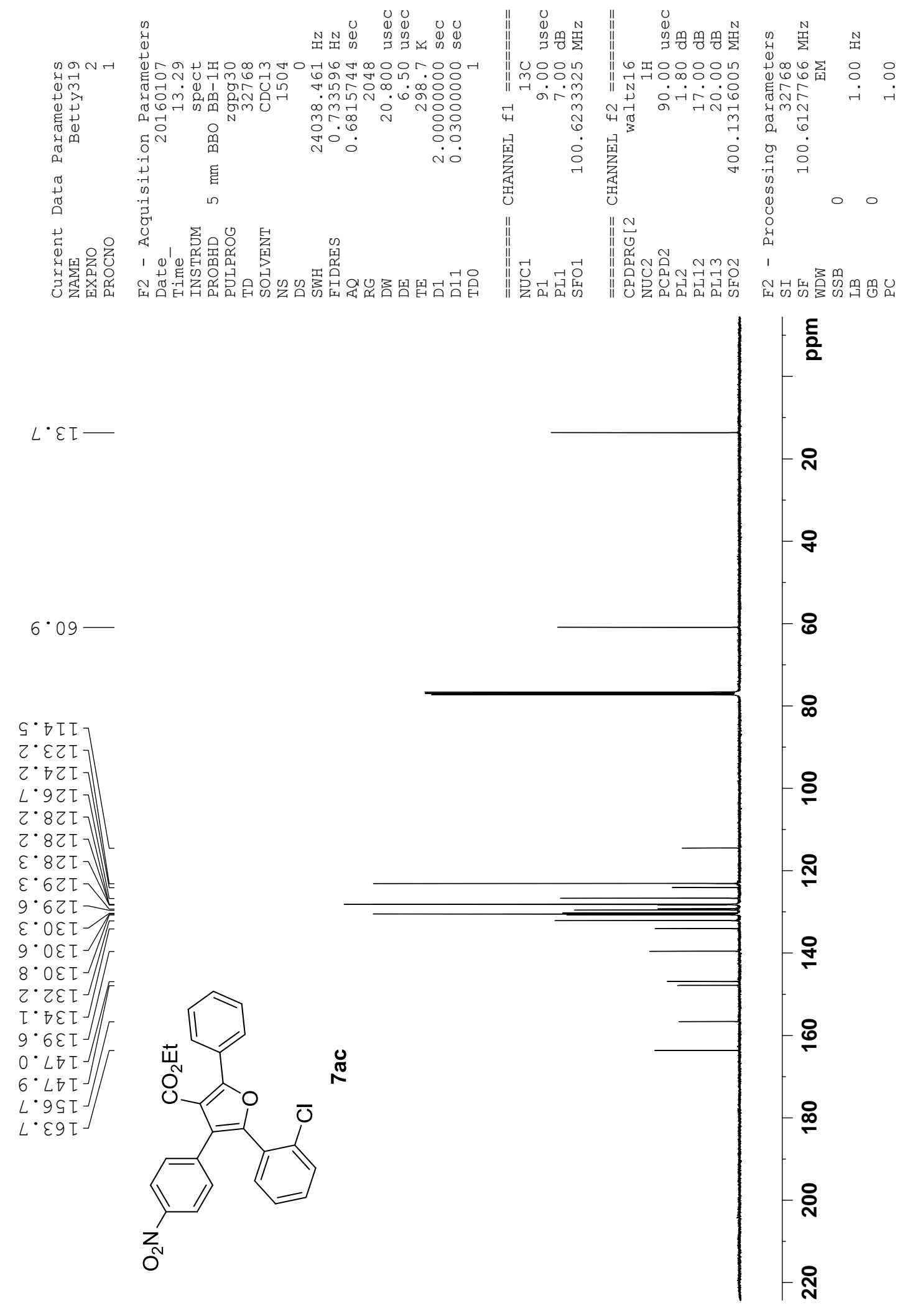




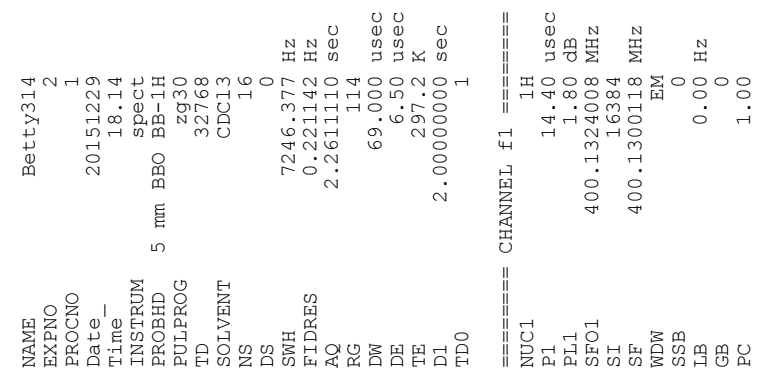

$86^{\circ} 0$
$66^{\circ}$
$[0$

位

$6 L \cdot \varepsilon>$

60
60

.

ZT.
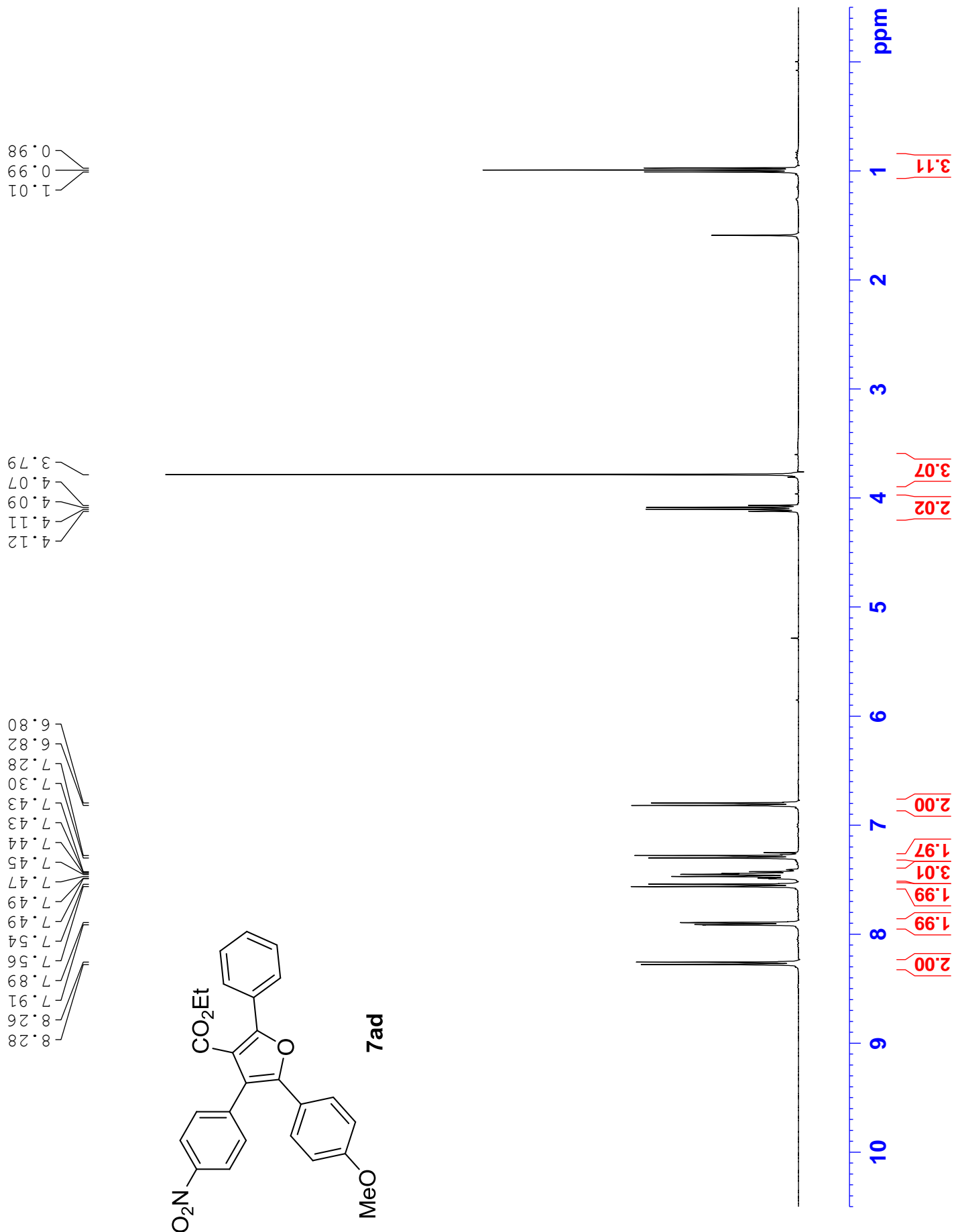


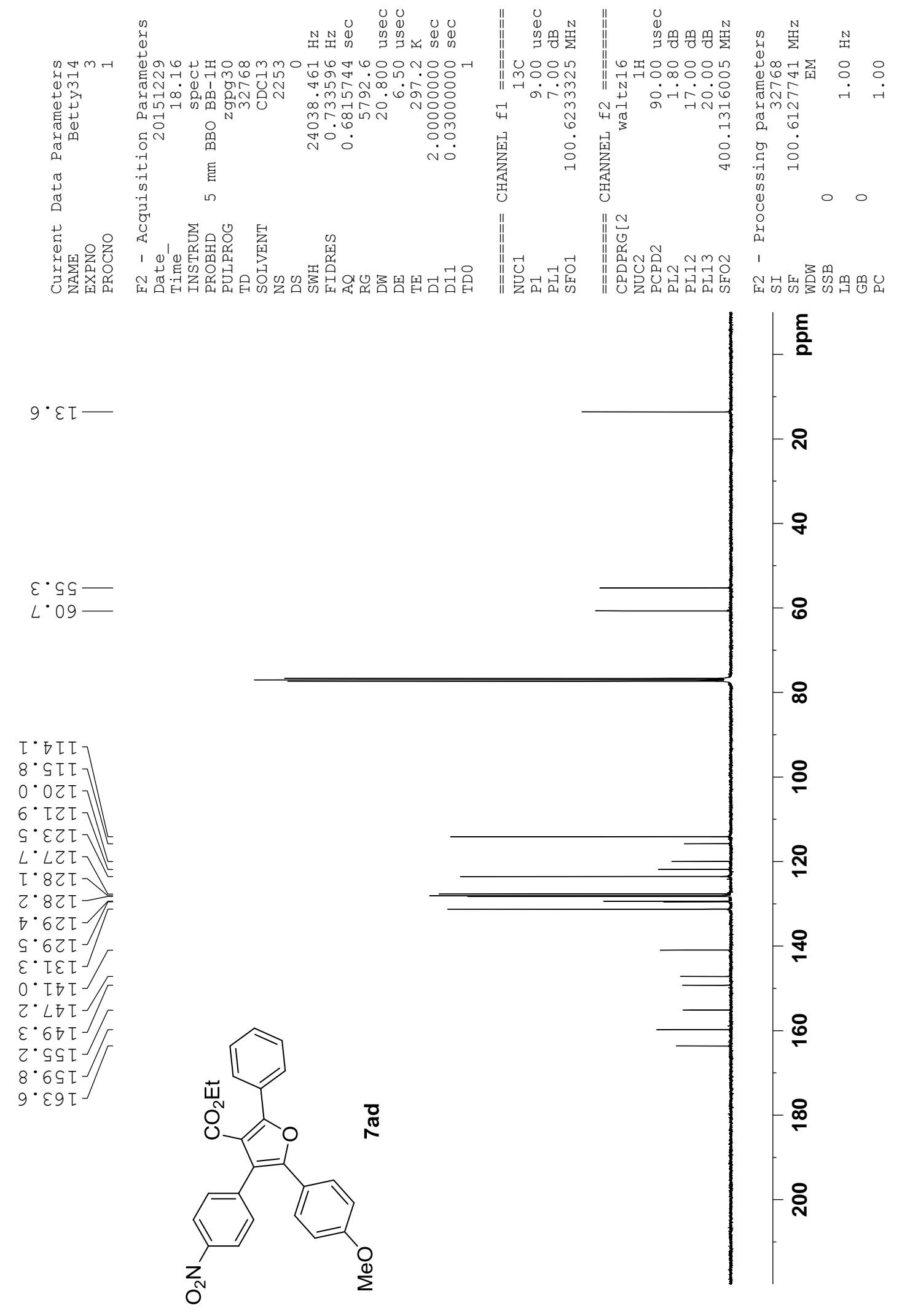




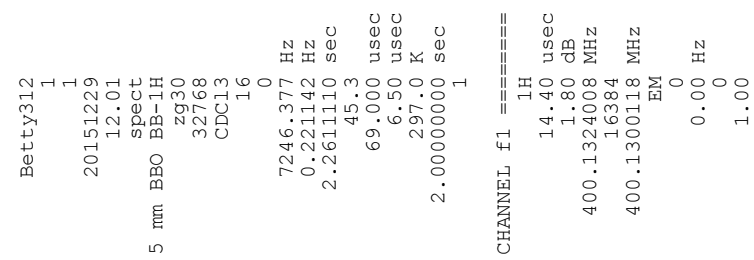

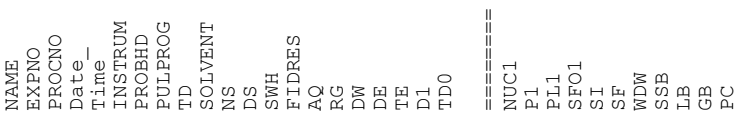

$86^{\circ}$
$00^{\circ}$

$\varepsilon \varepsilon \cdot \tau$

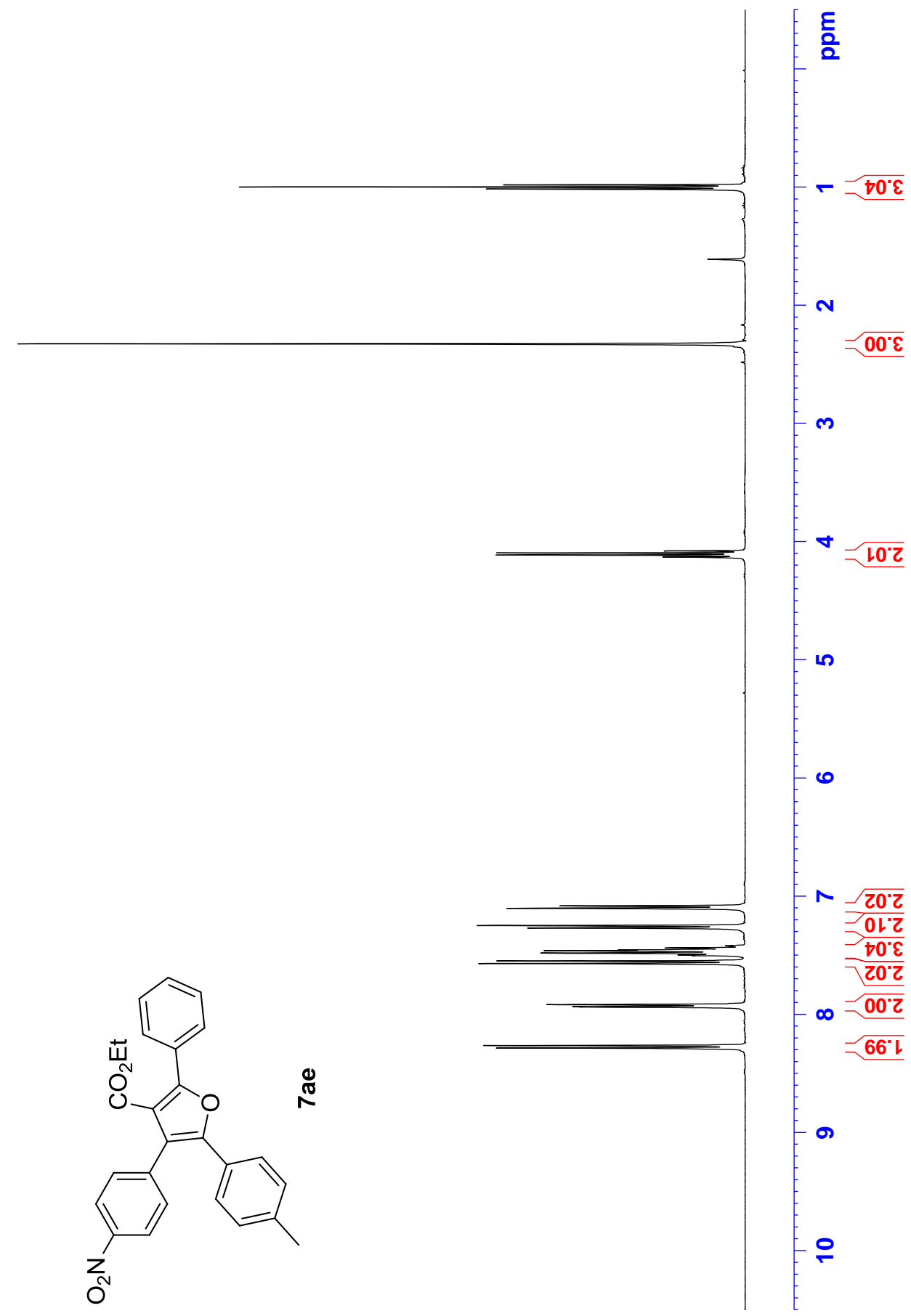

$80^{\circ} \cdot 7$

$0 \tau \cdot \bar{T}$

乙I.

$\varepsilon T \cdot$

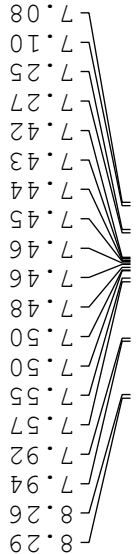



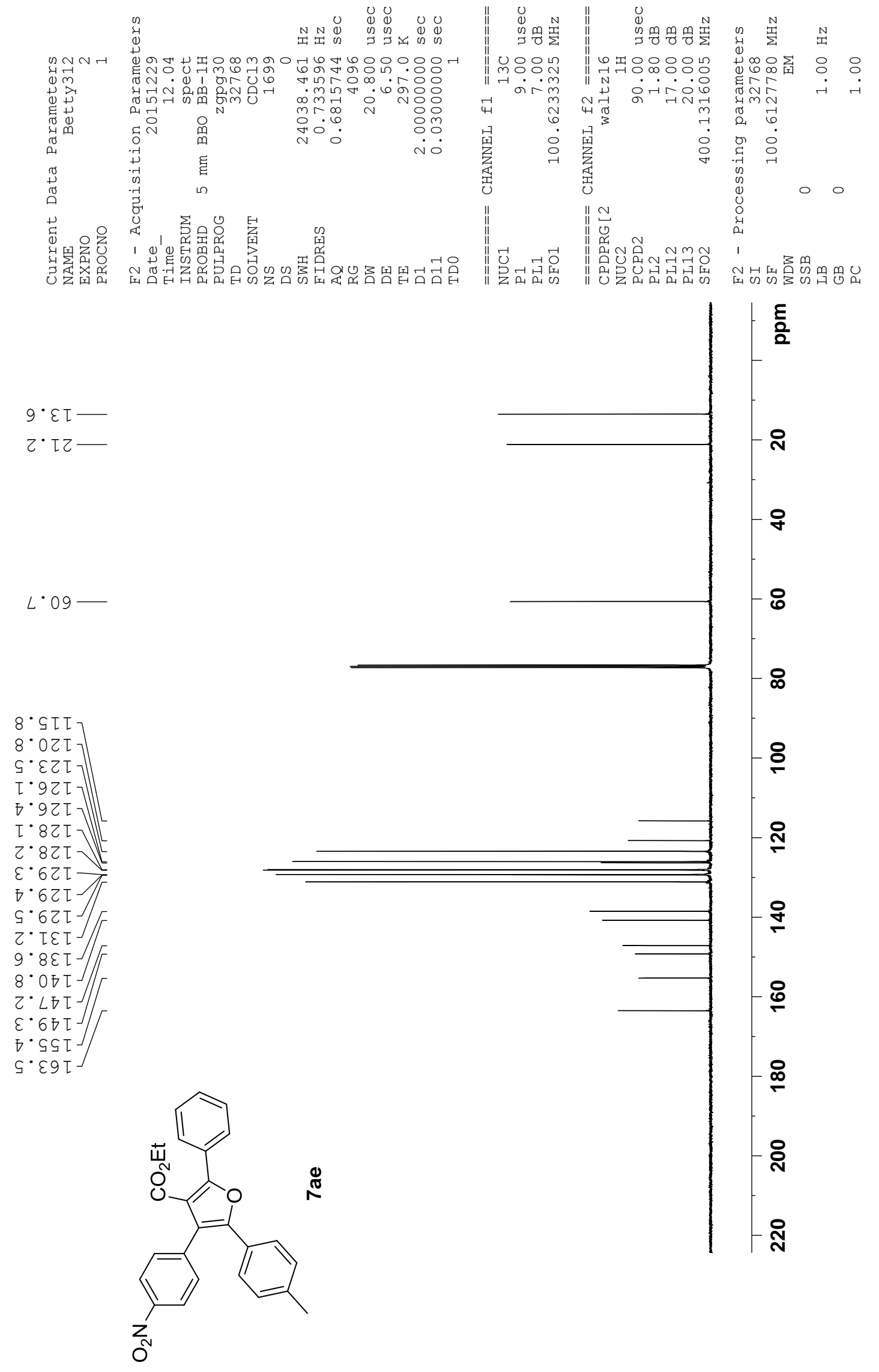


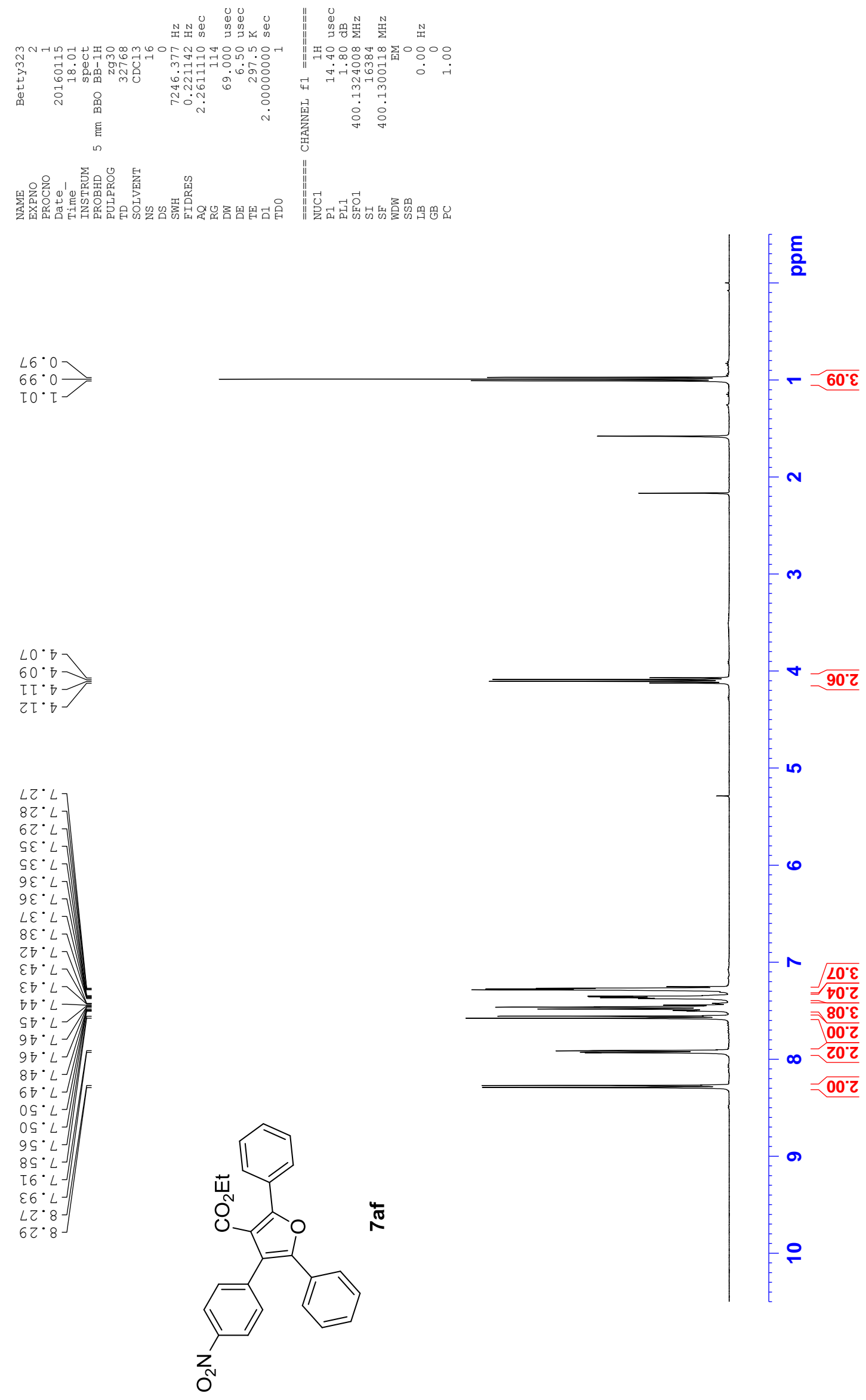




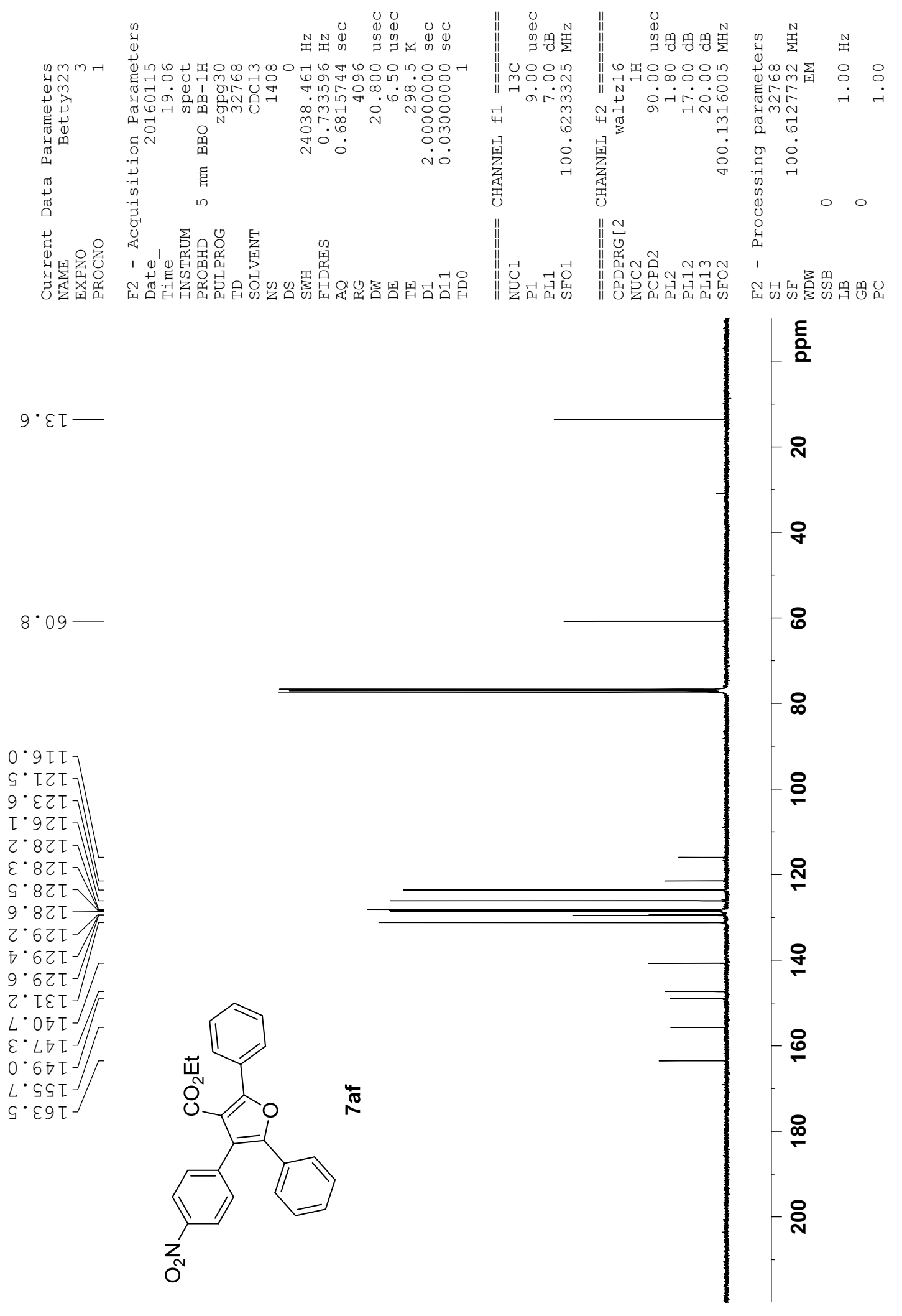




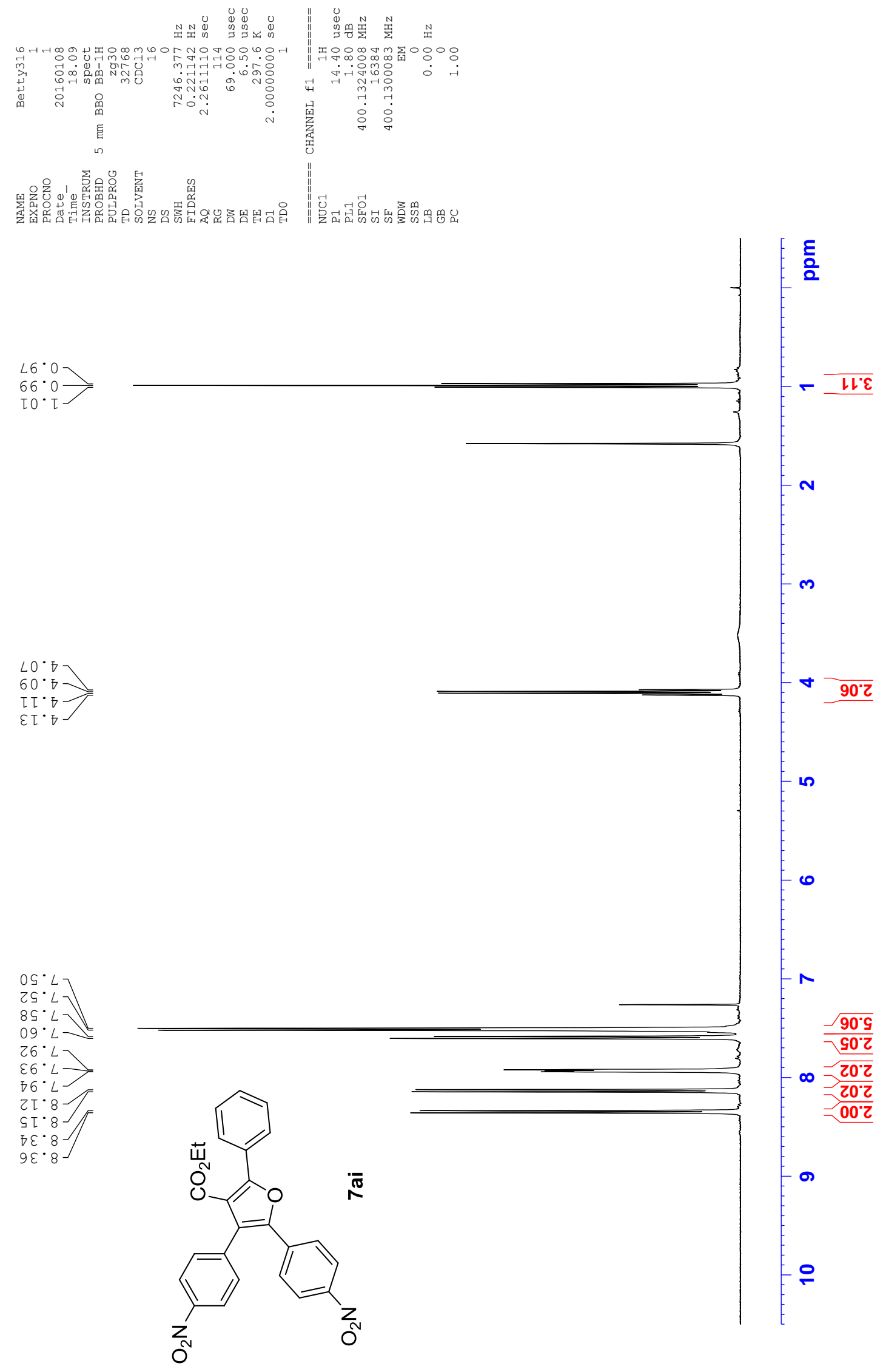




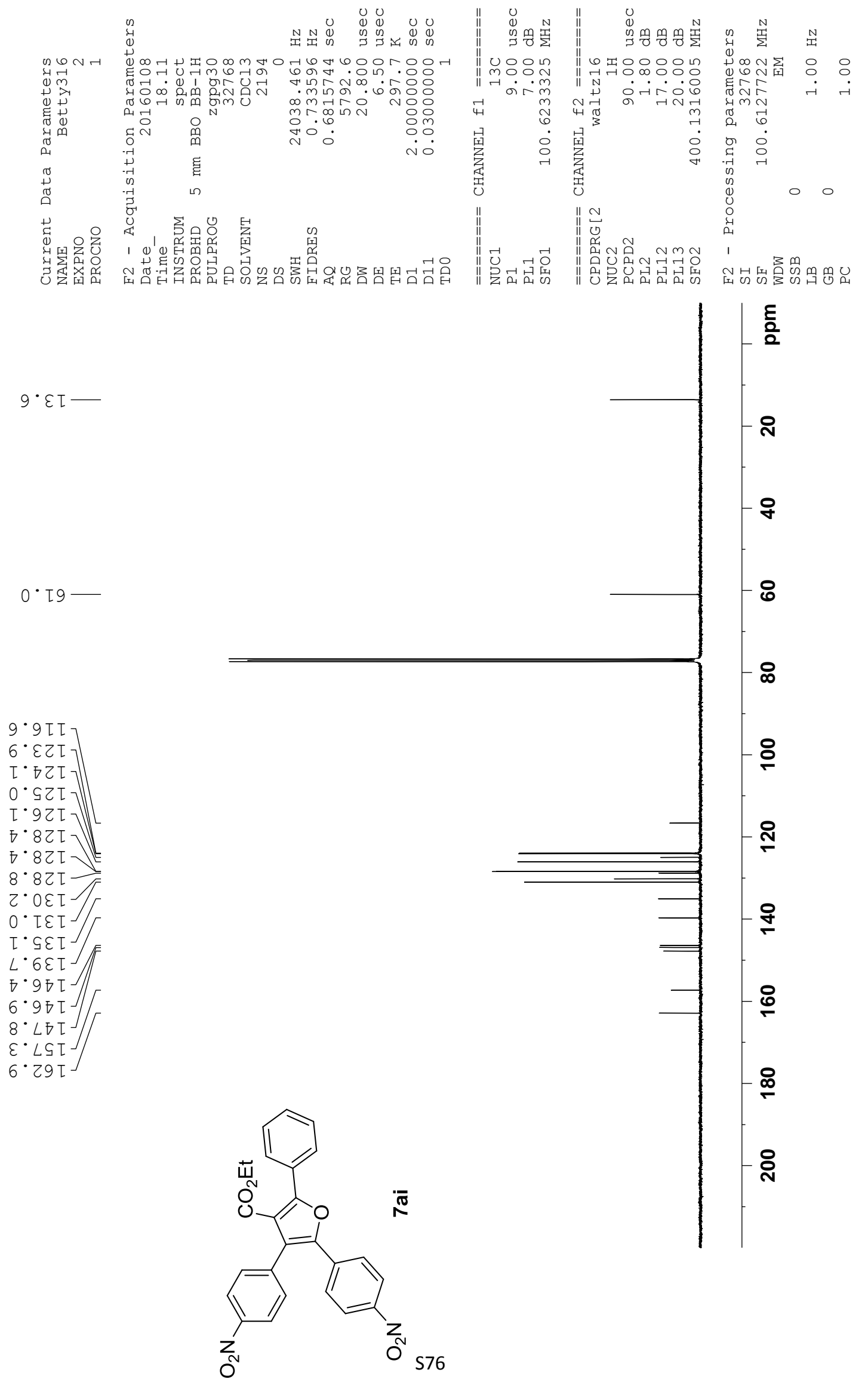




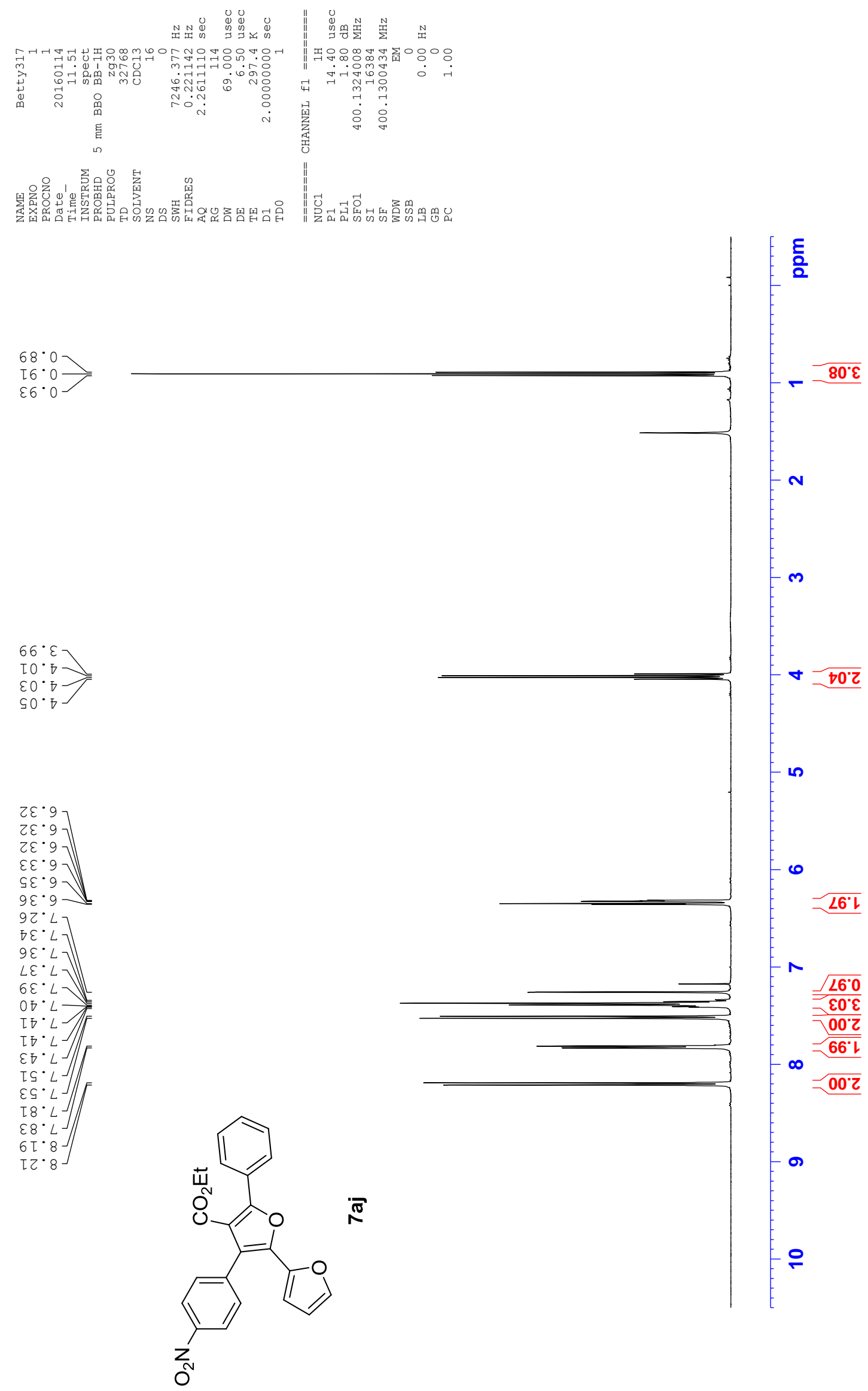




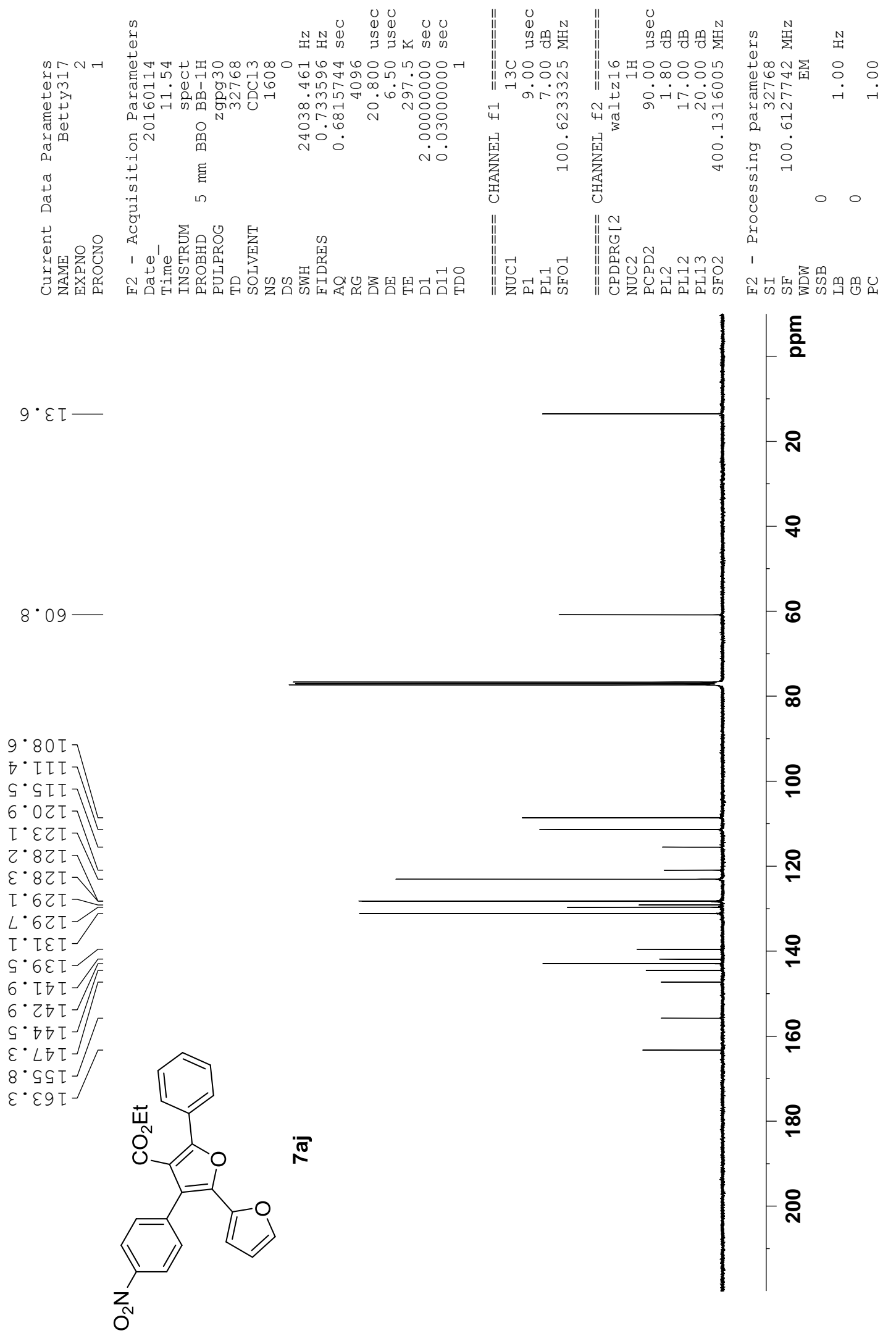



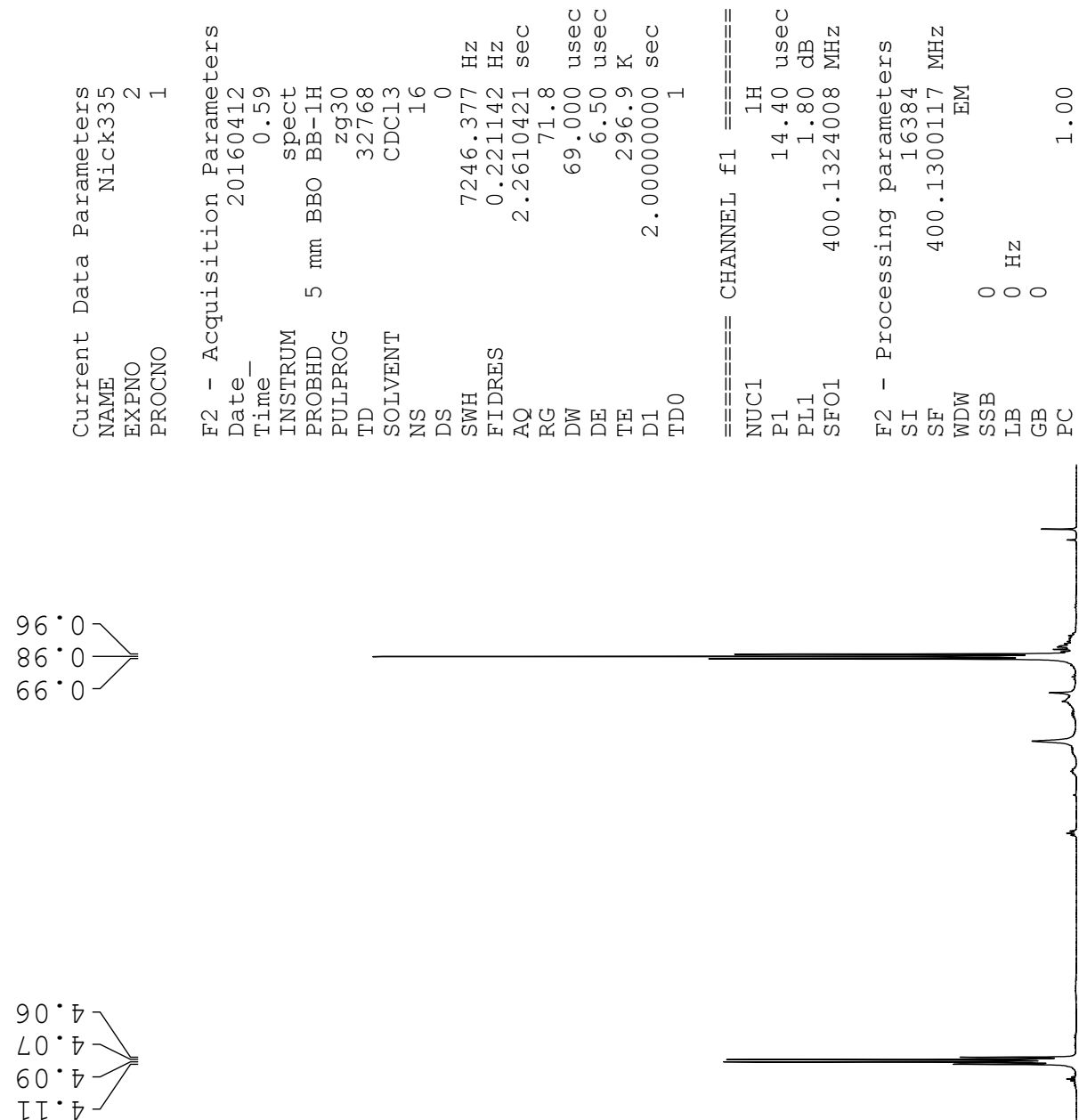

E 틍
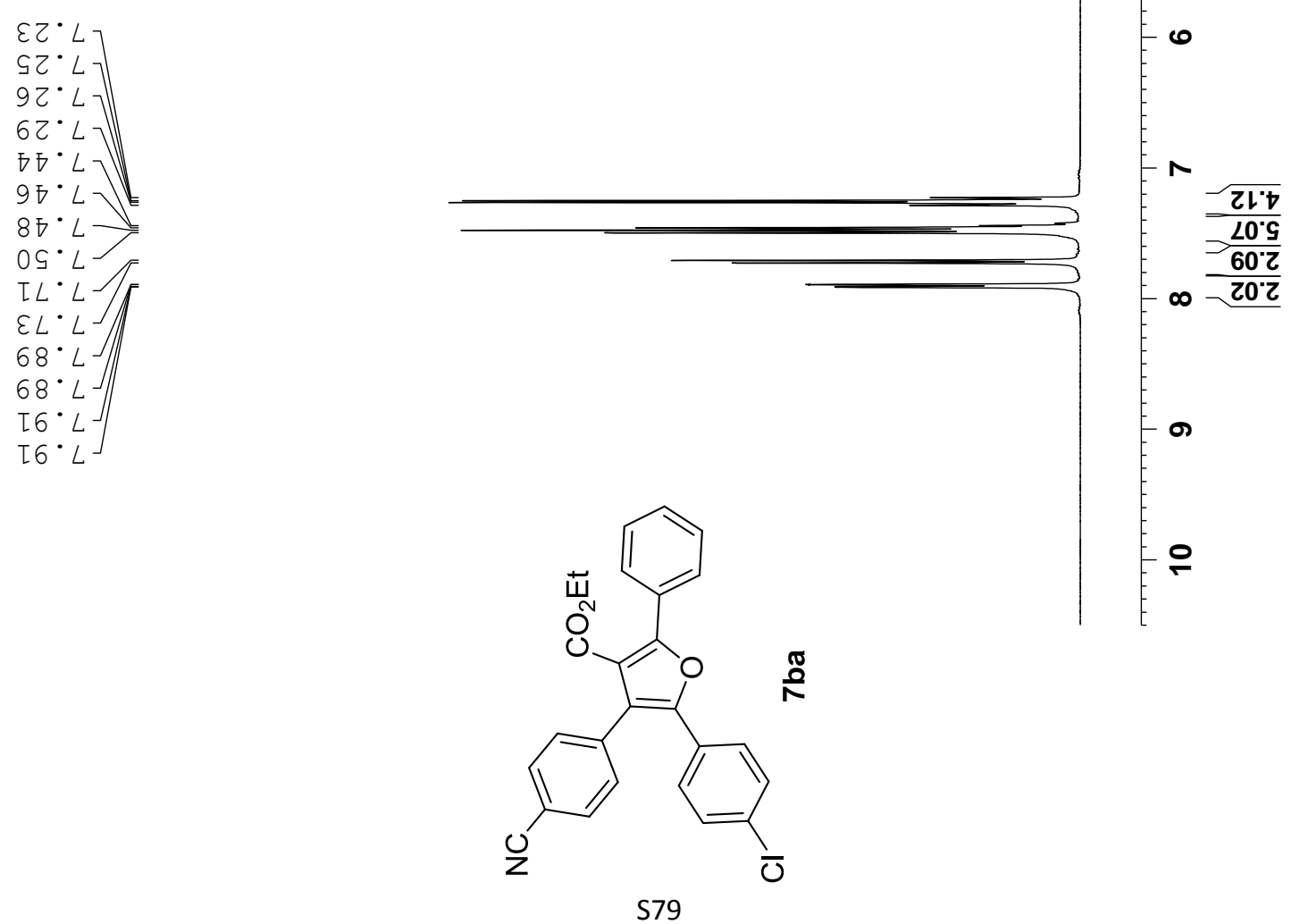

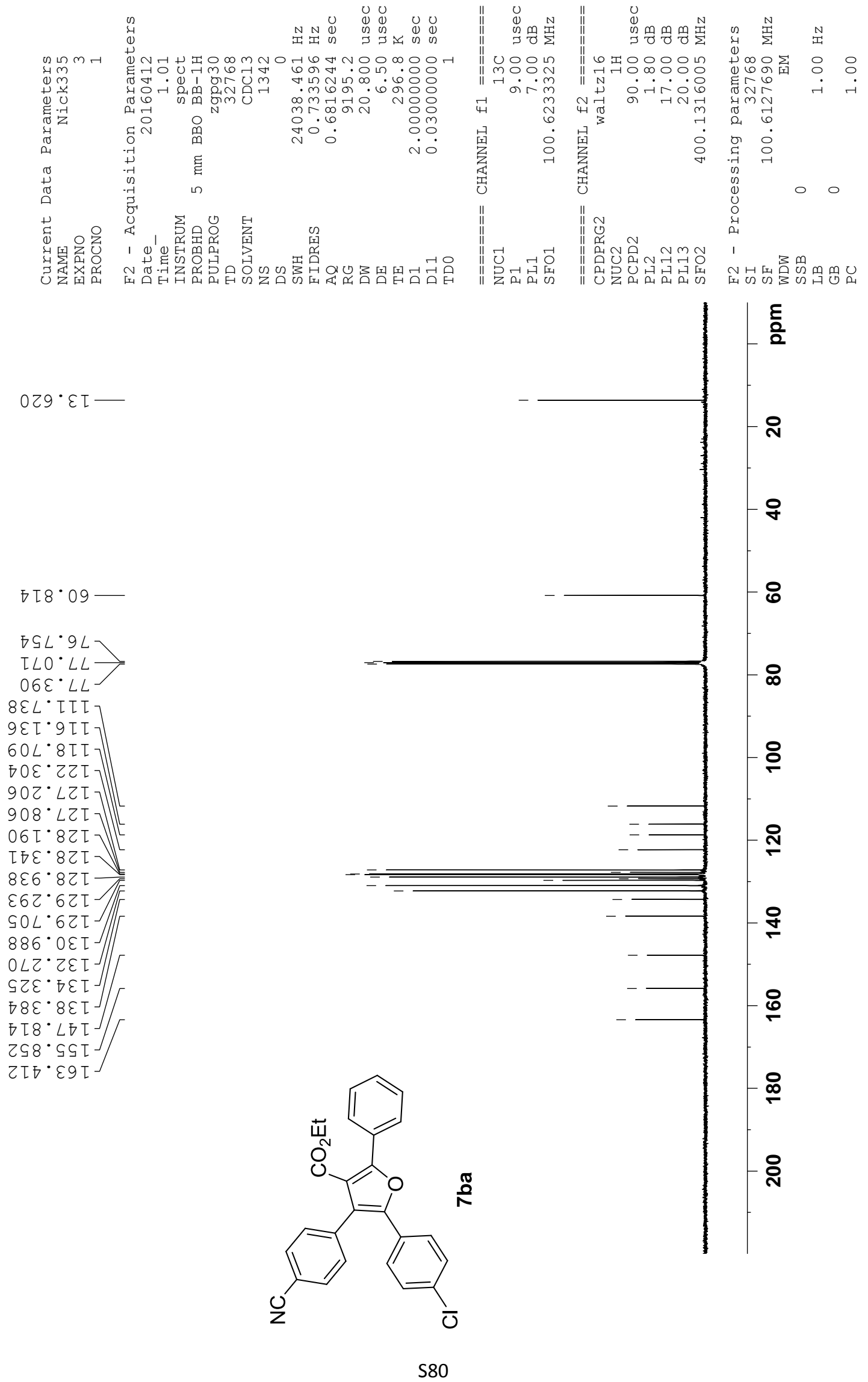

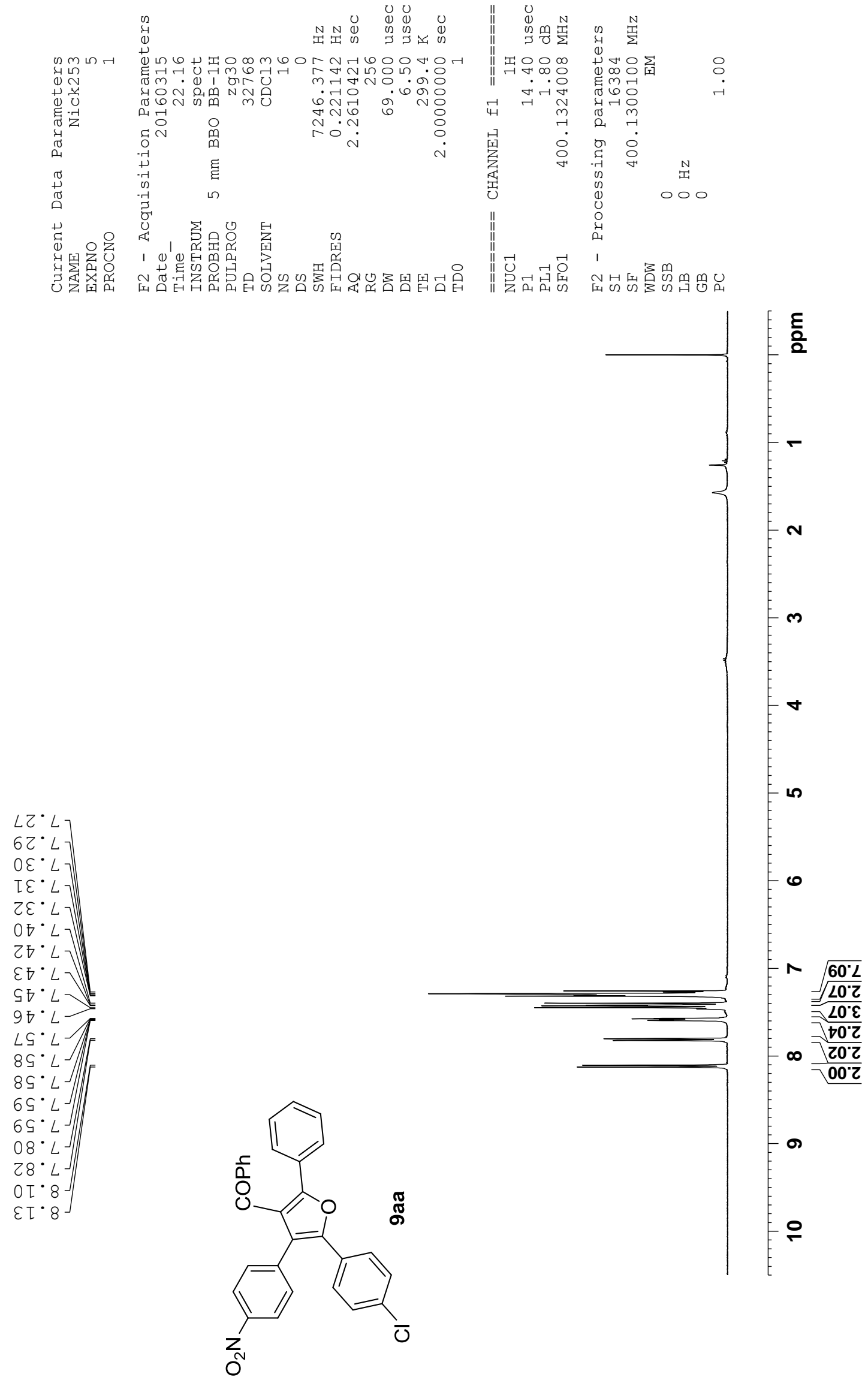

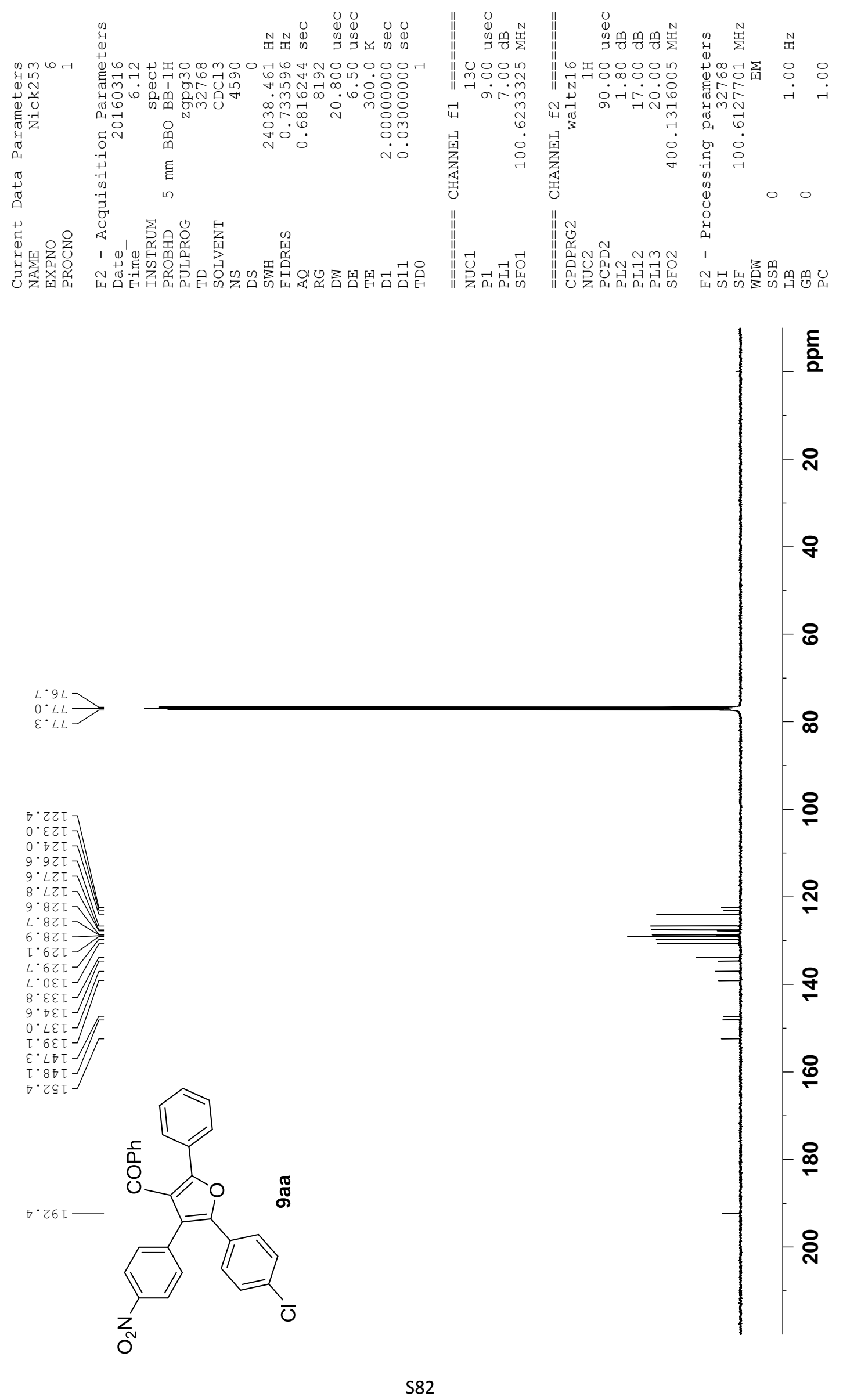

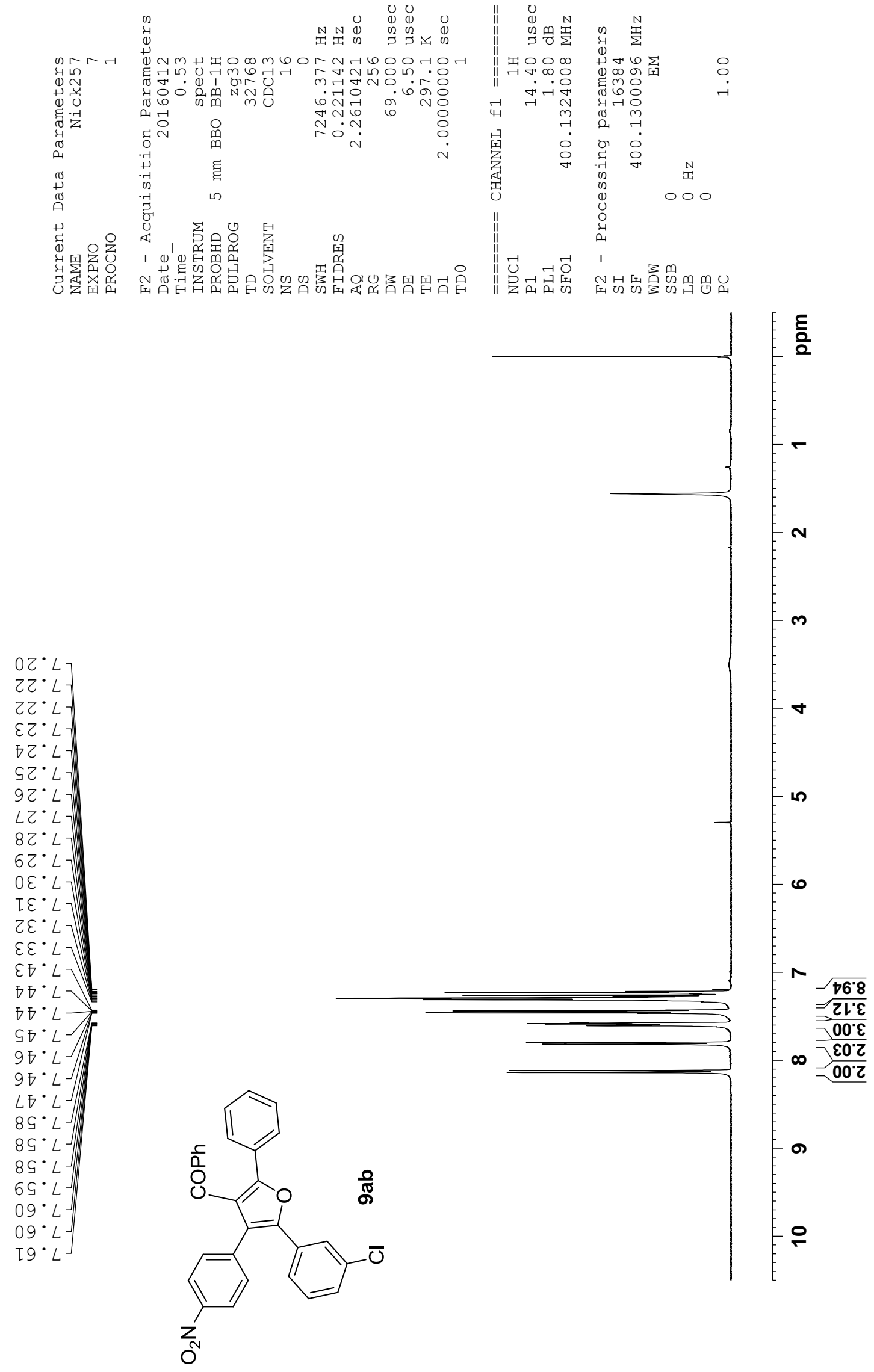

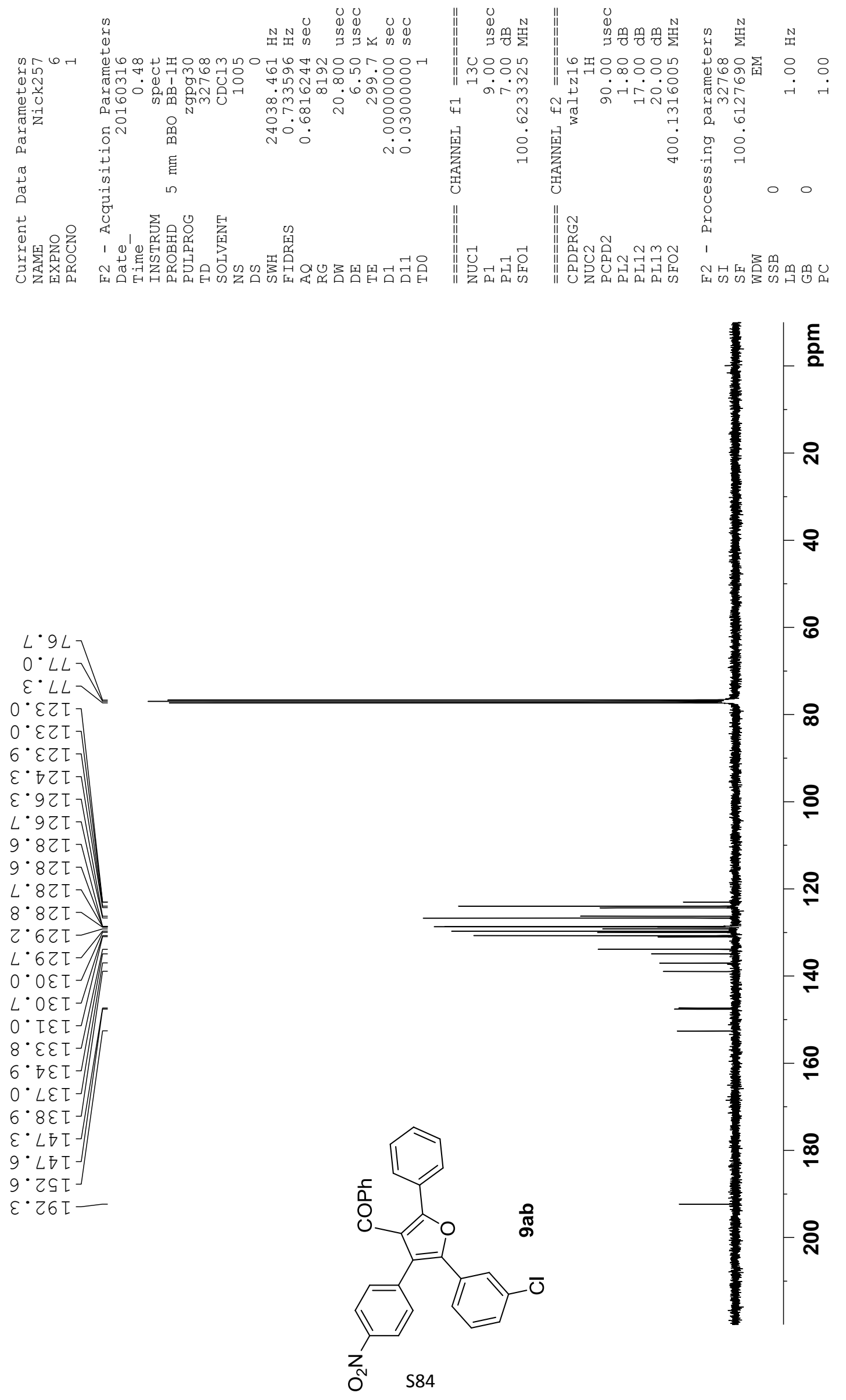


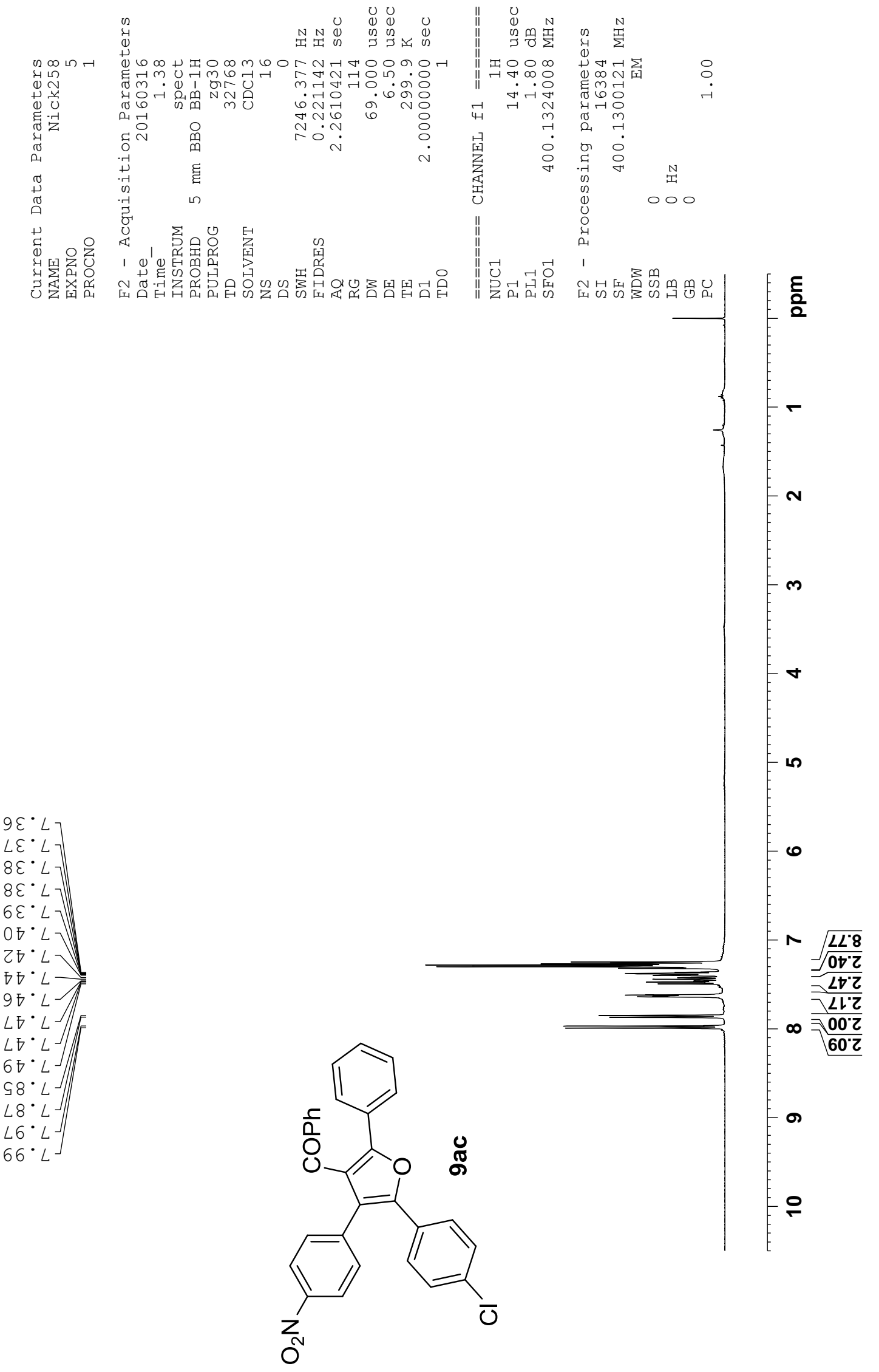



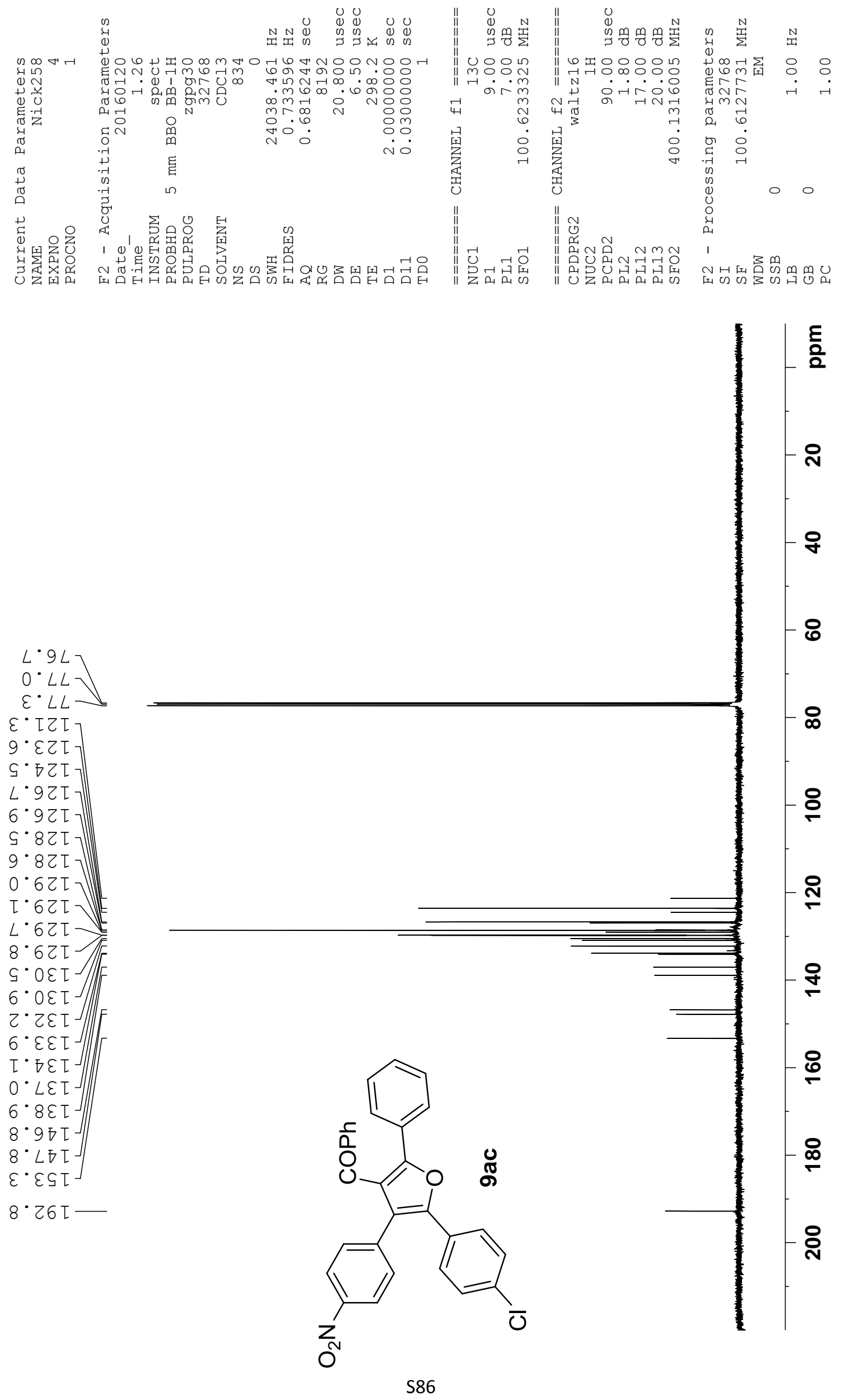

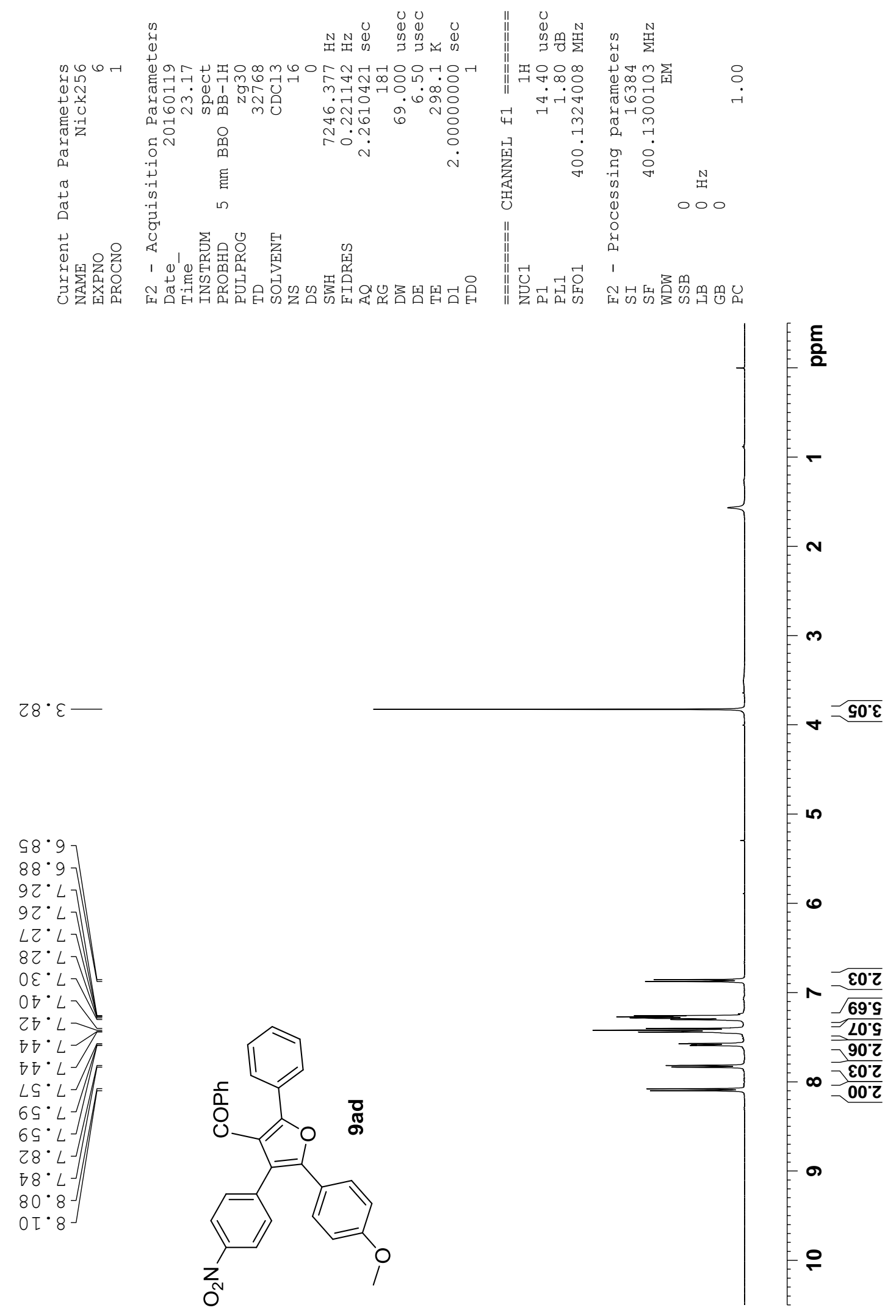

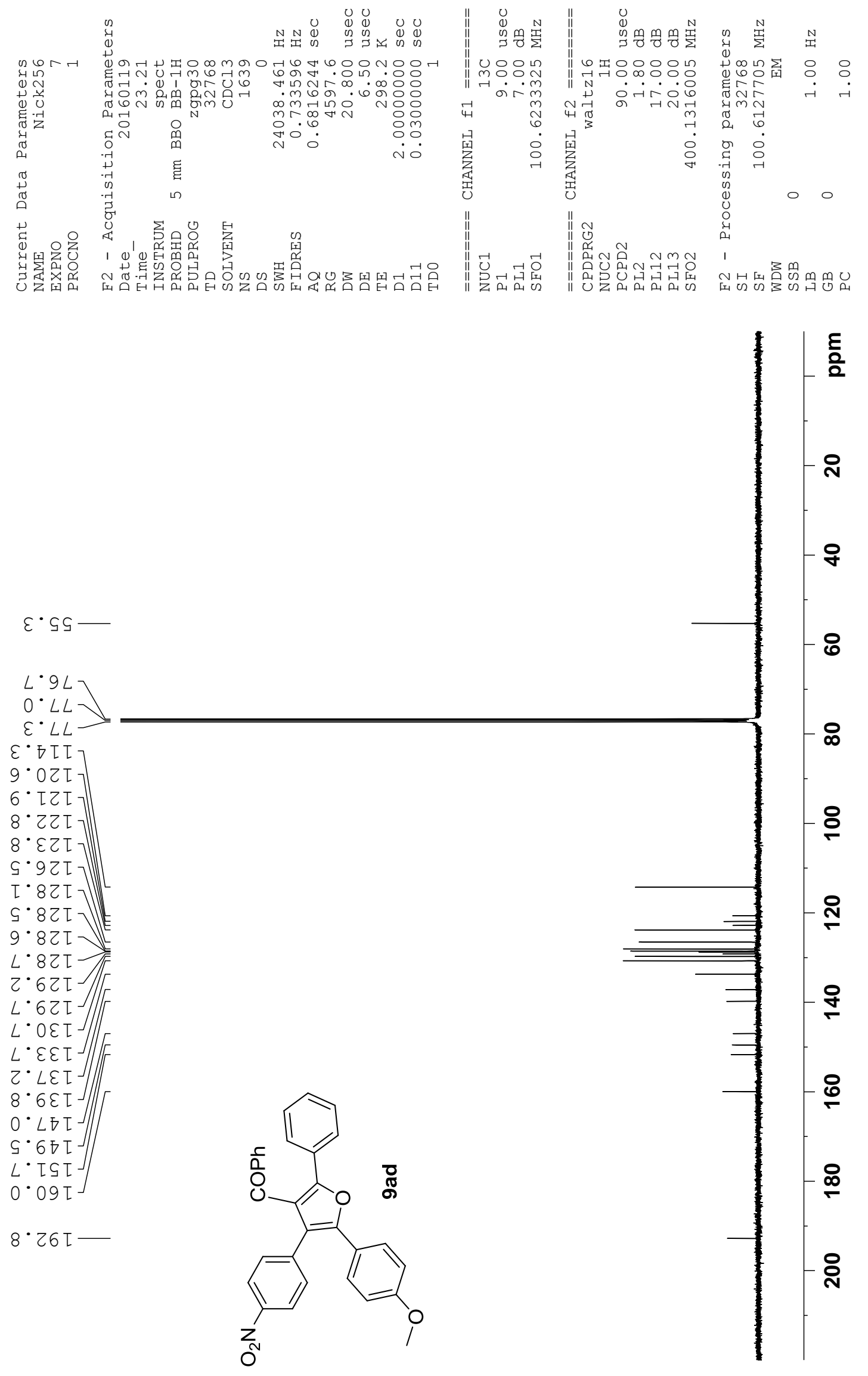

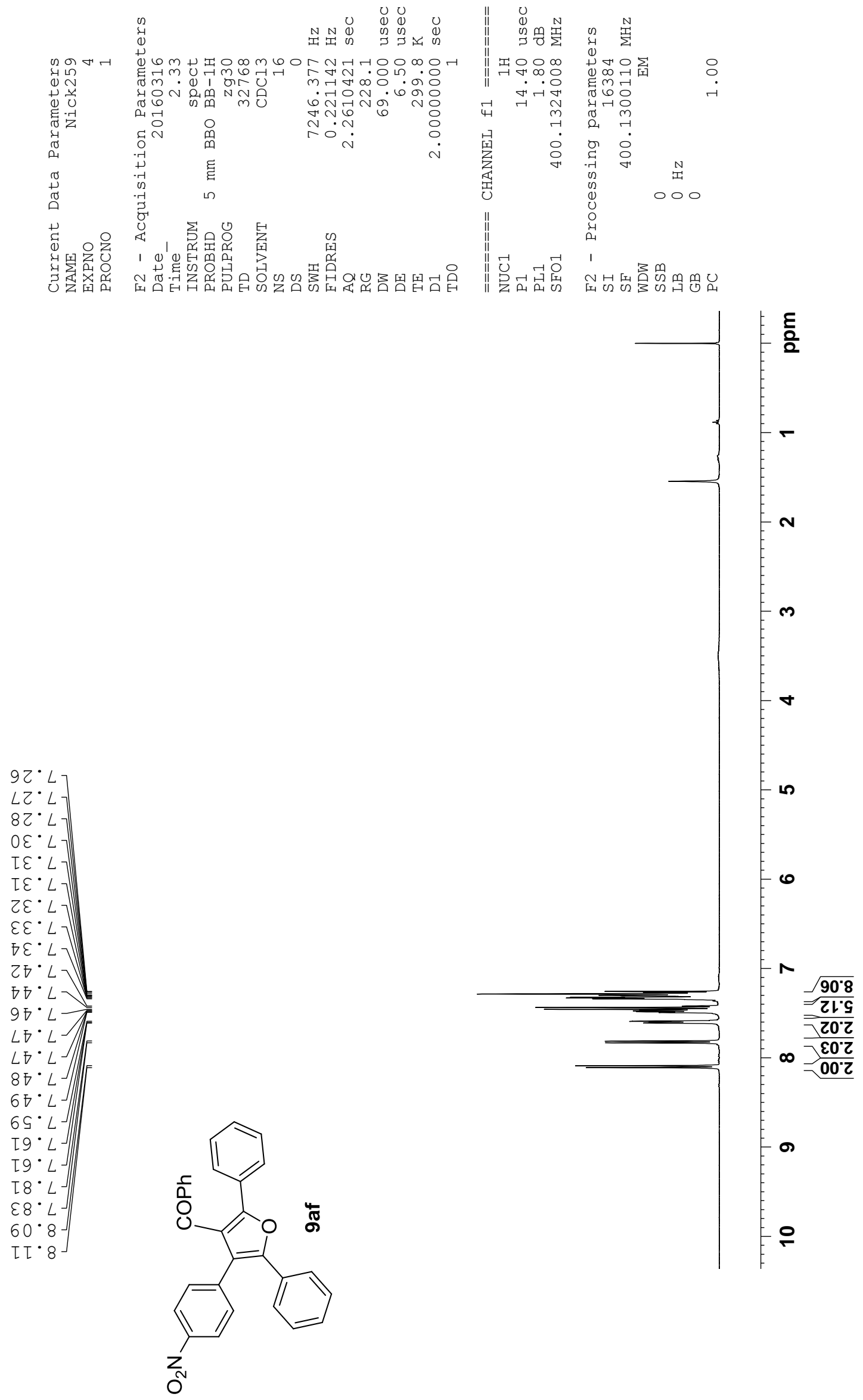

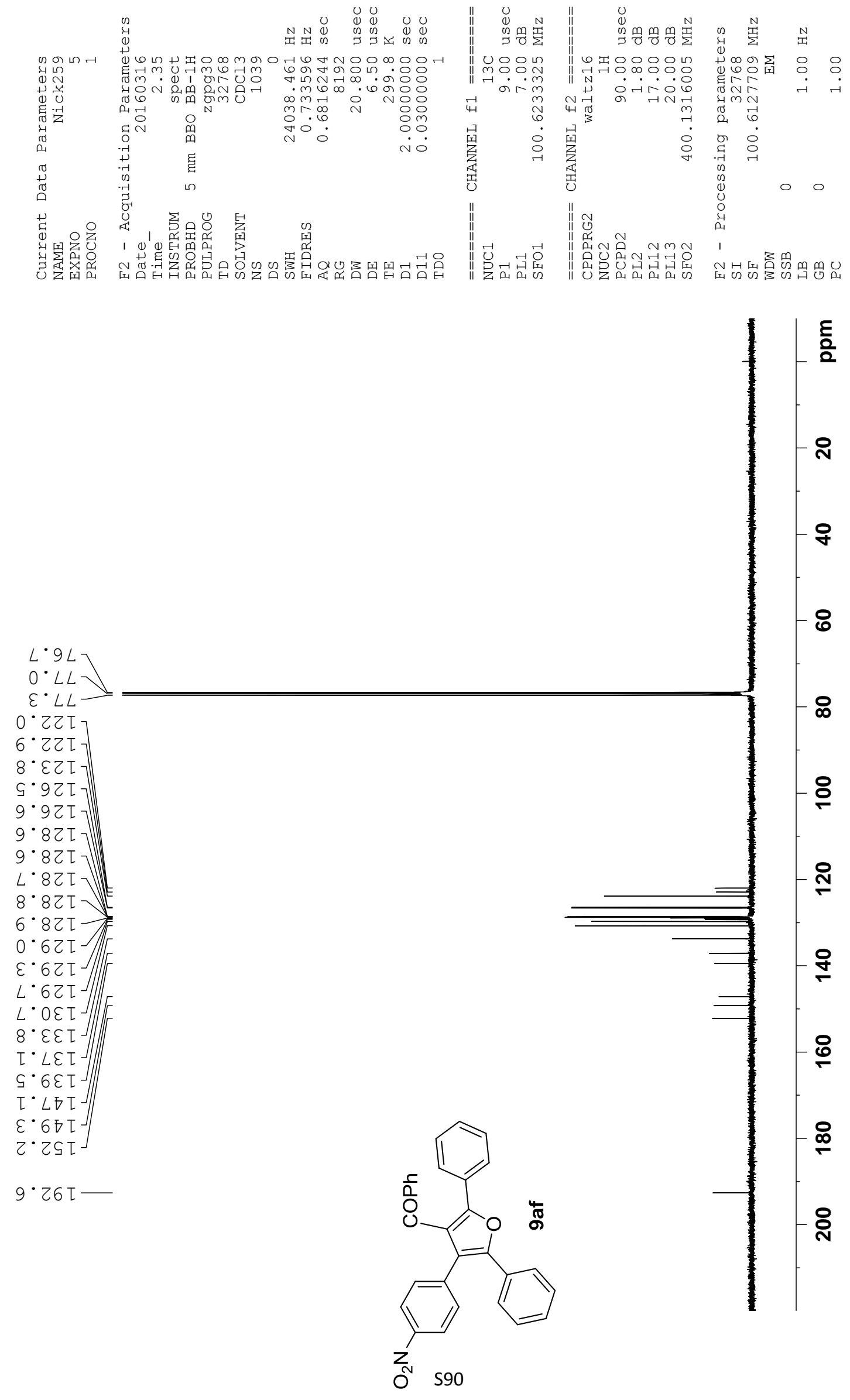


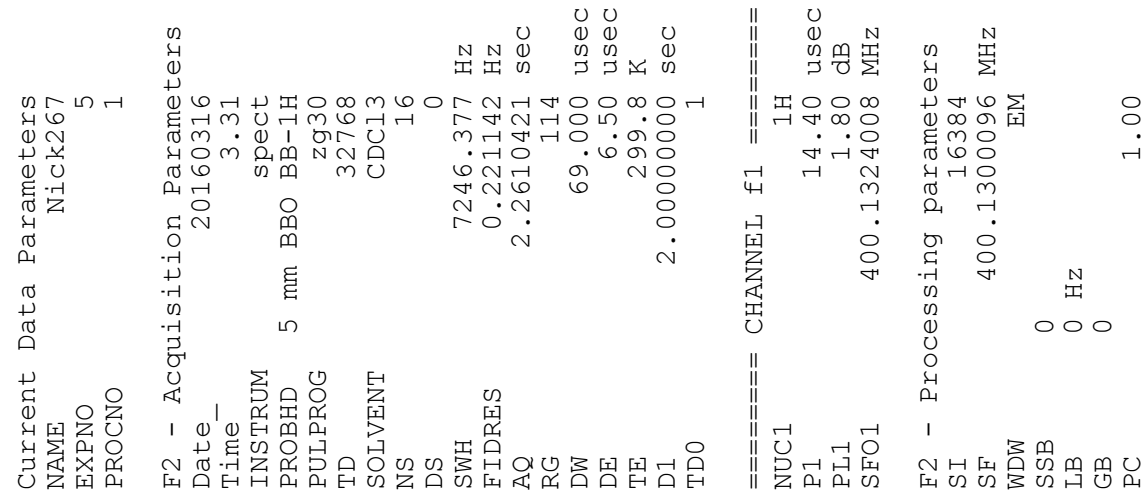
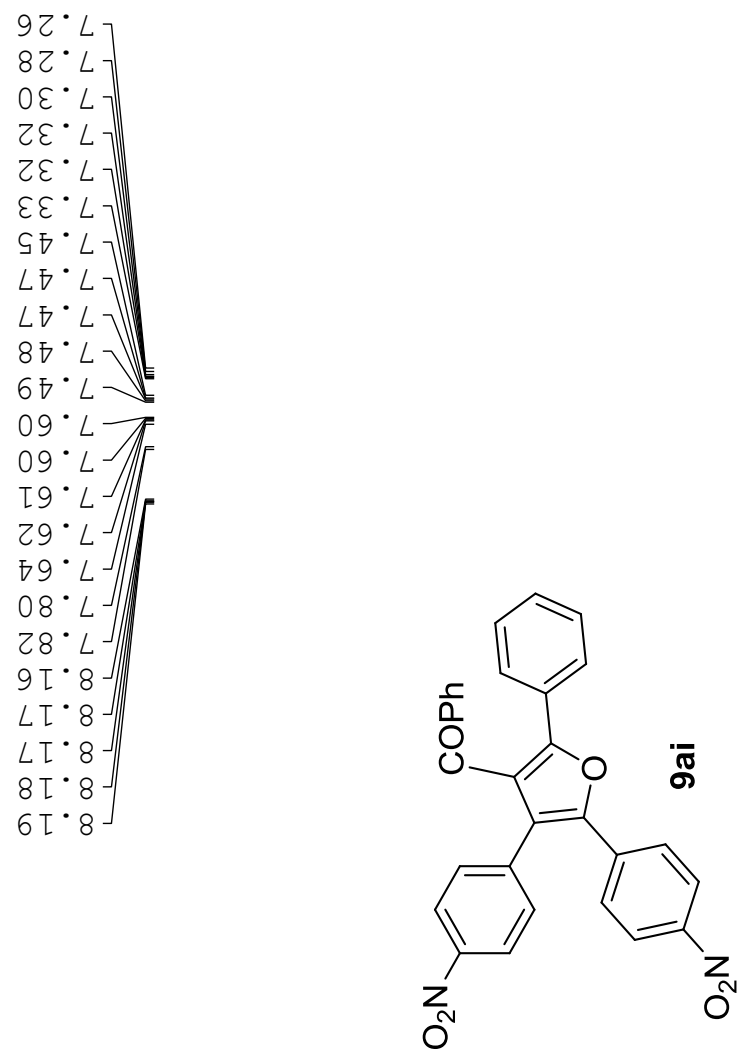

$a$ 

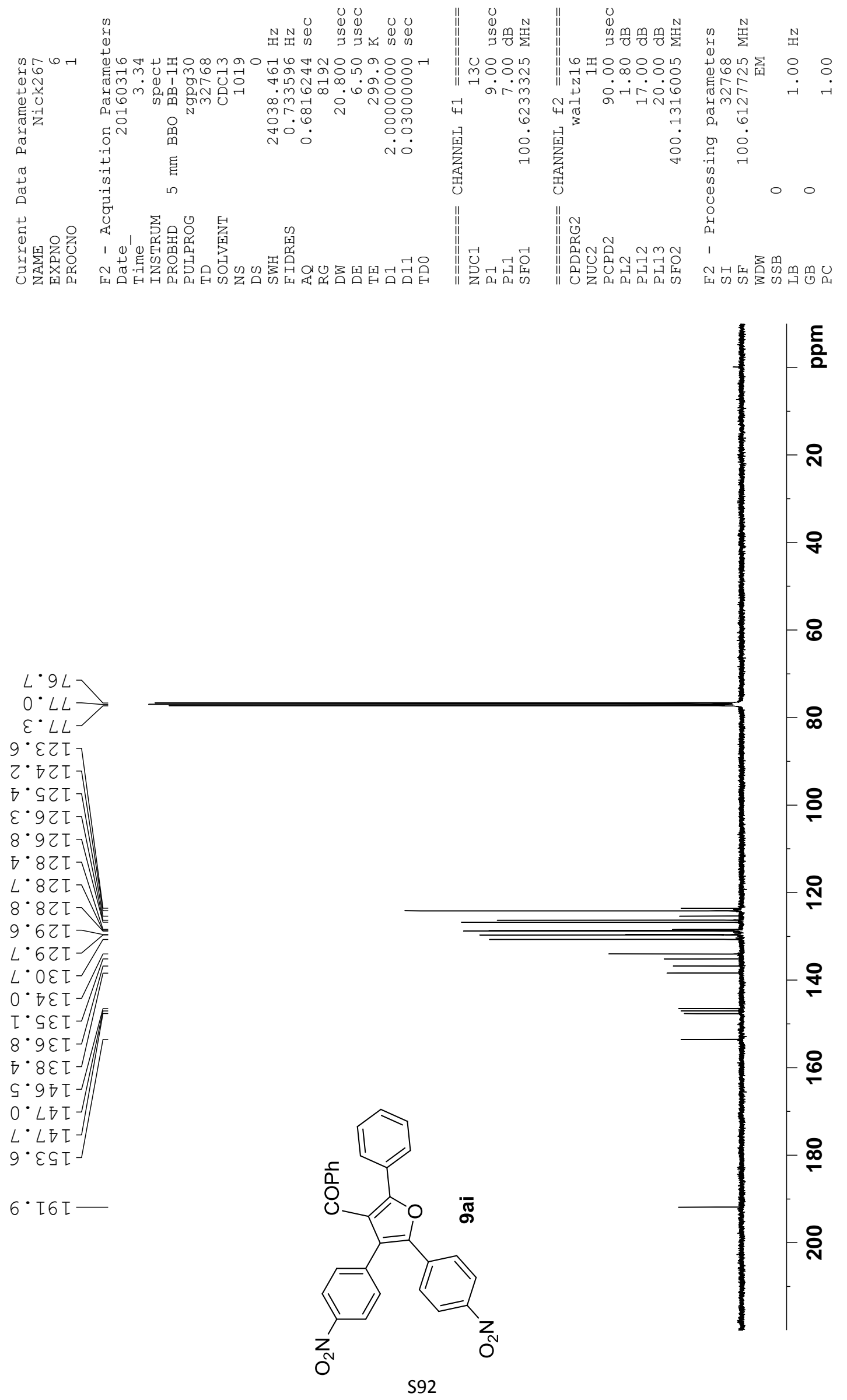

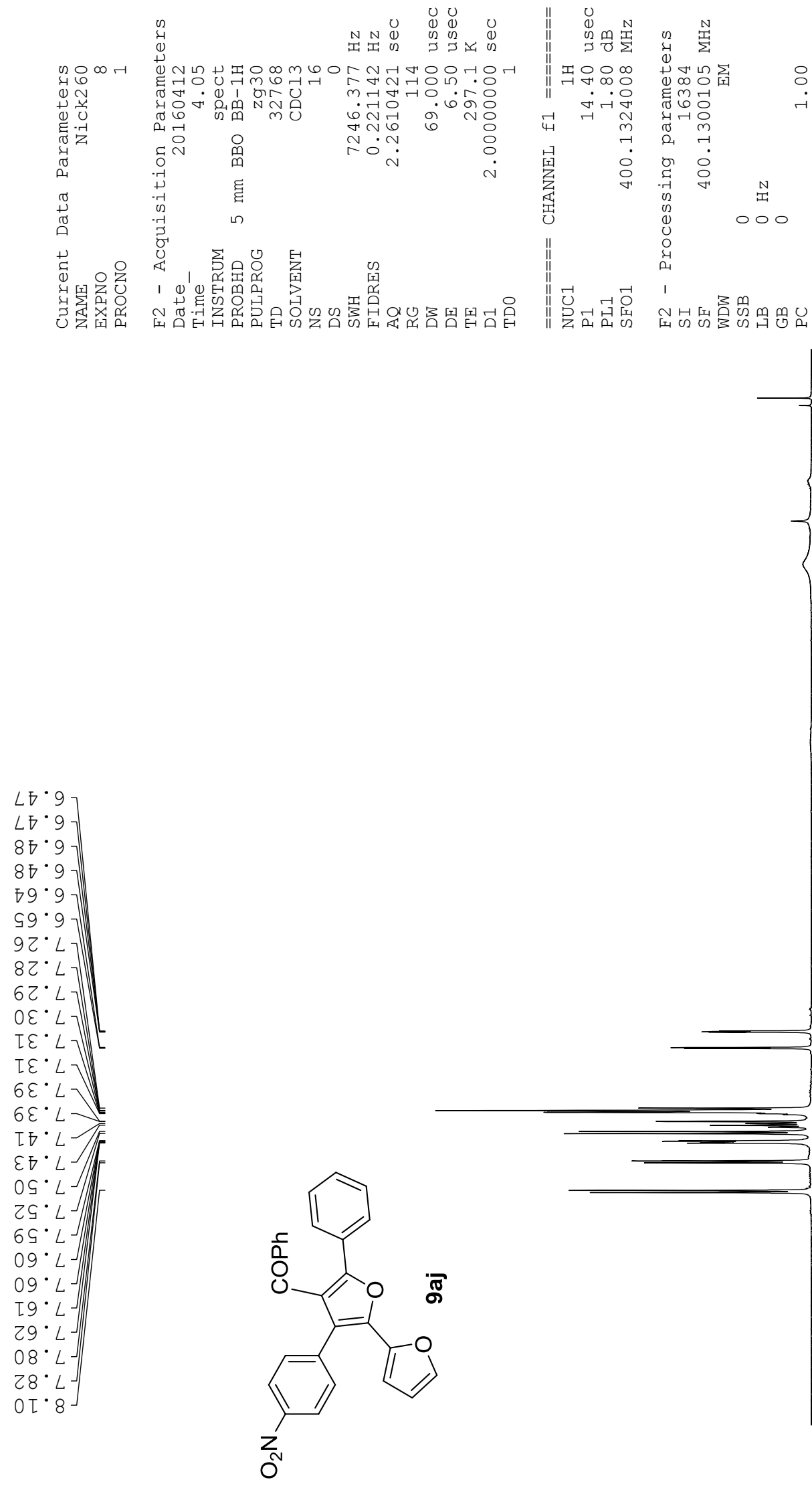

흘

$N$

$m$

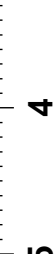

in

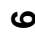

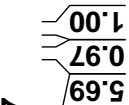

$\wedge \quad 69$

t5.1

$8 L^{\prime \prime 2}$

$\sqrt{2 L C}$

$\sqrt{90^{\circ} Z}$

LL'

の

으 

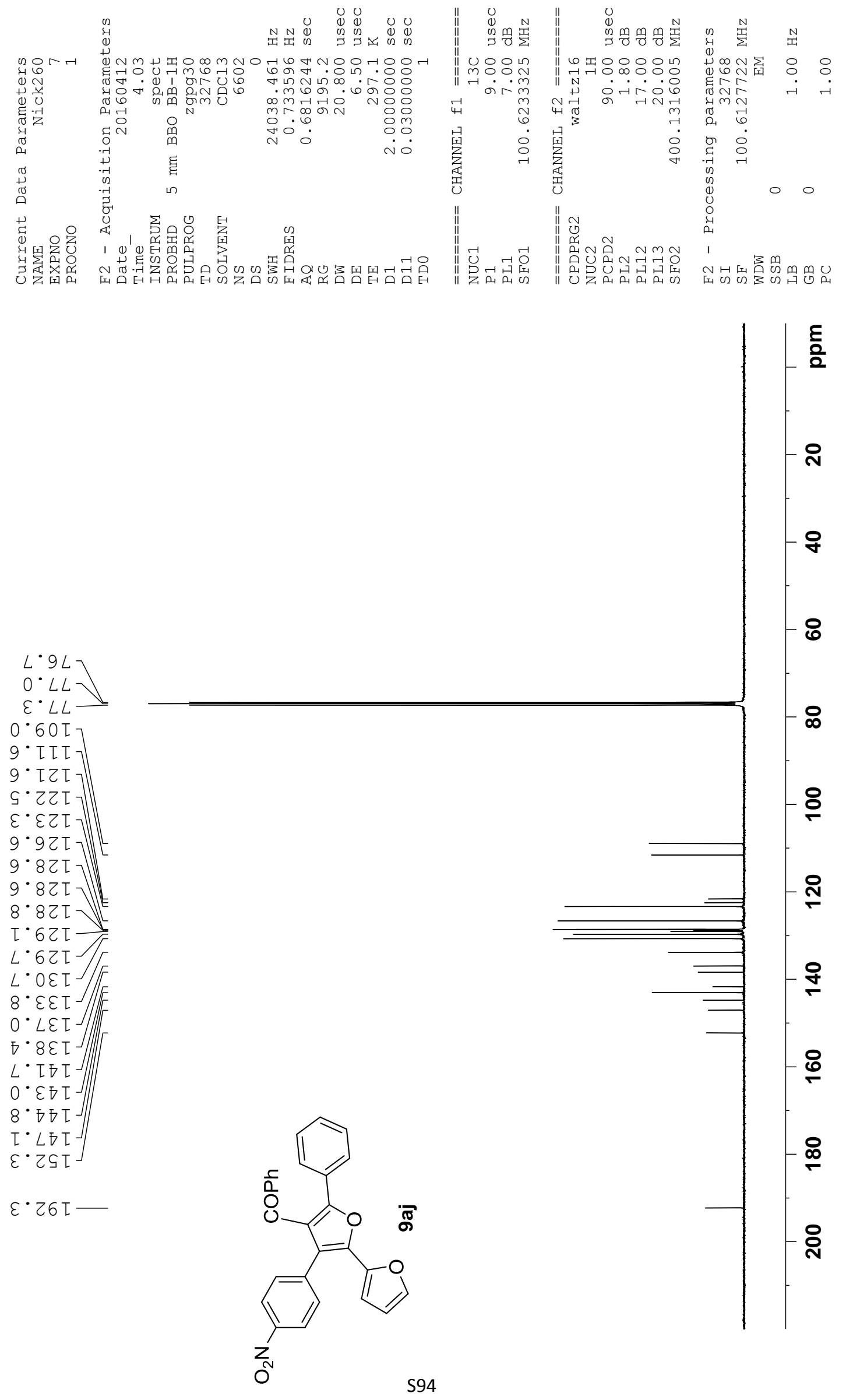

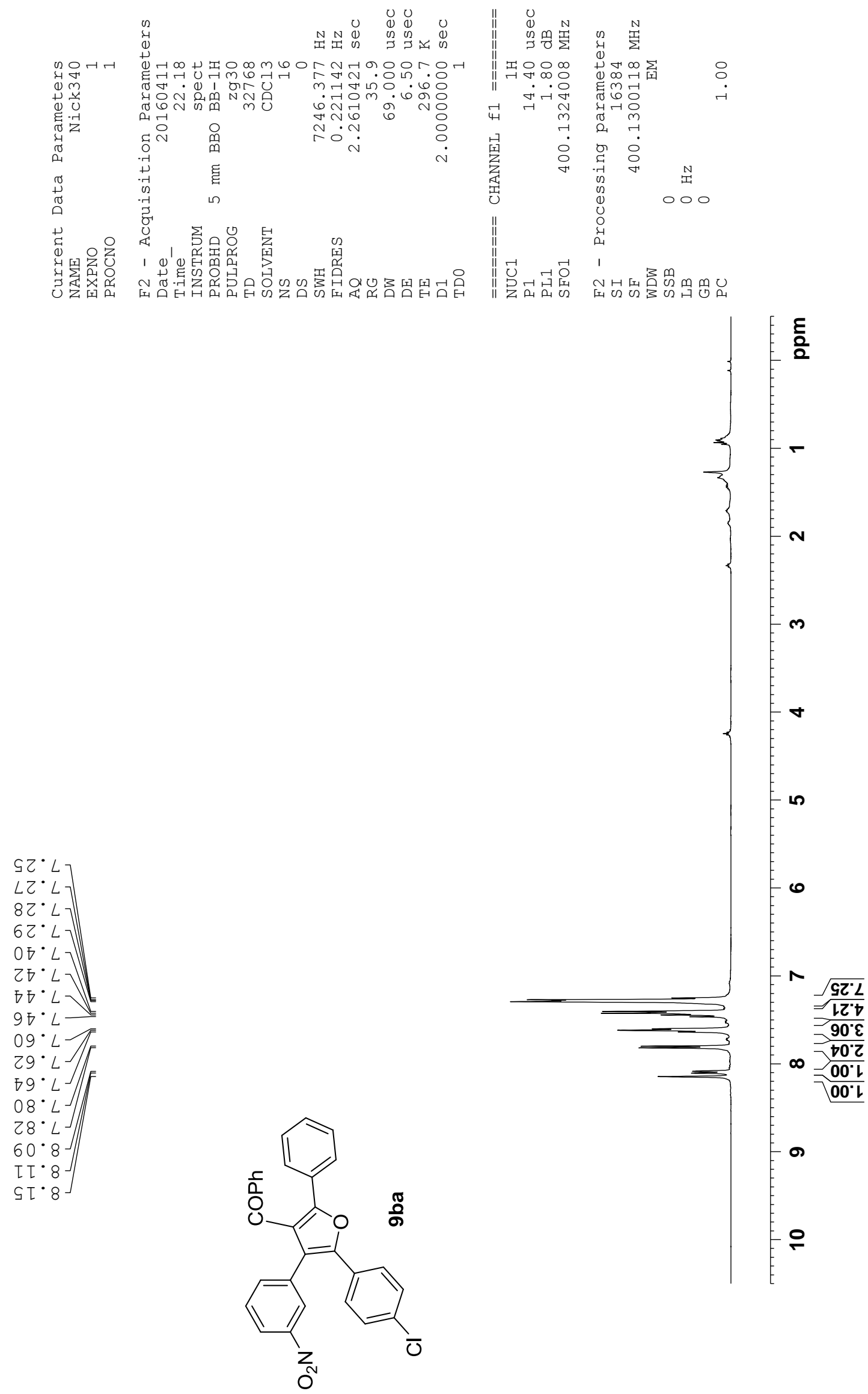

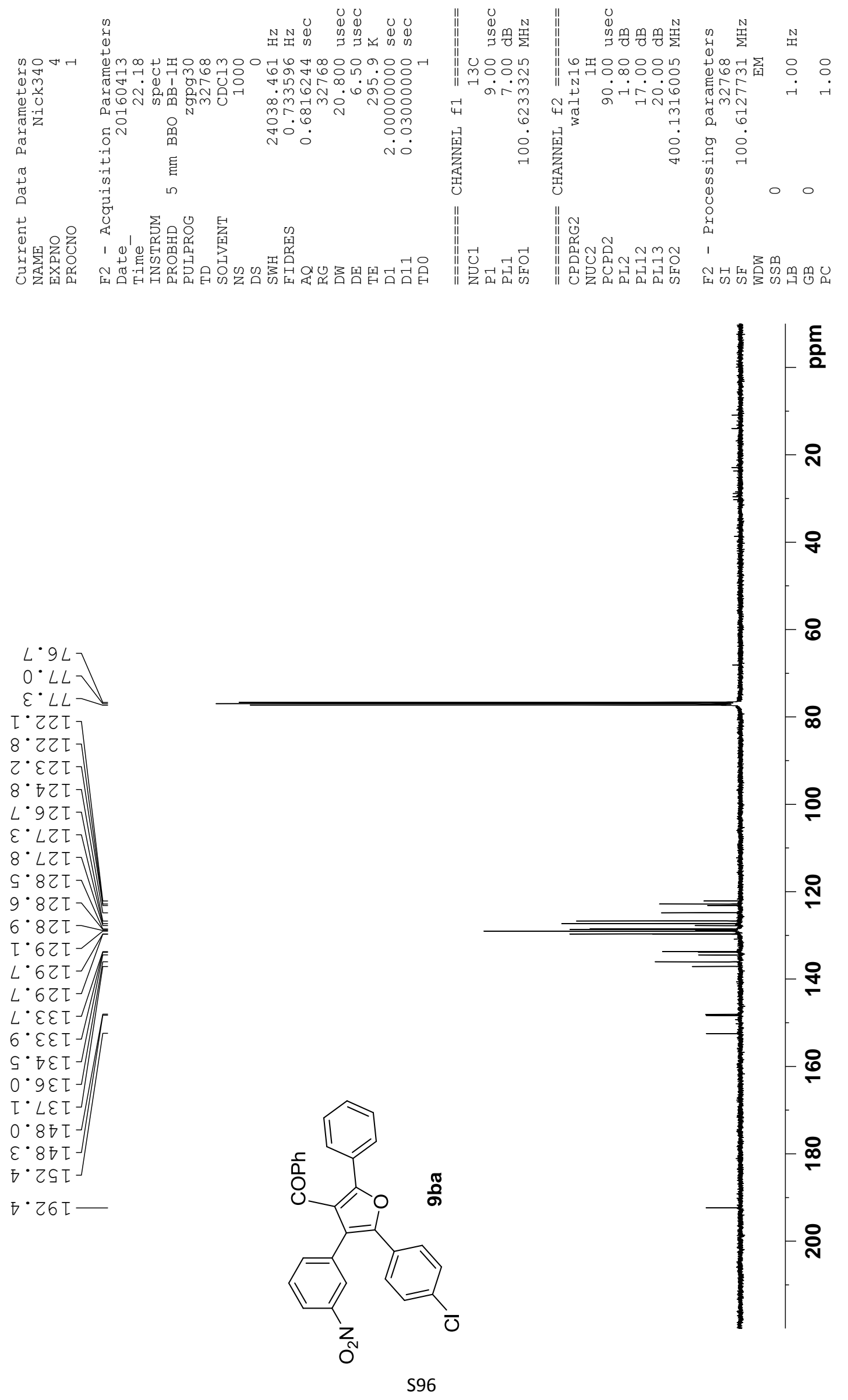

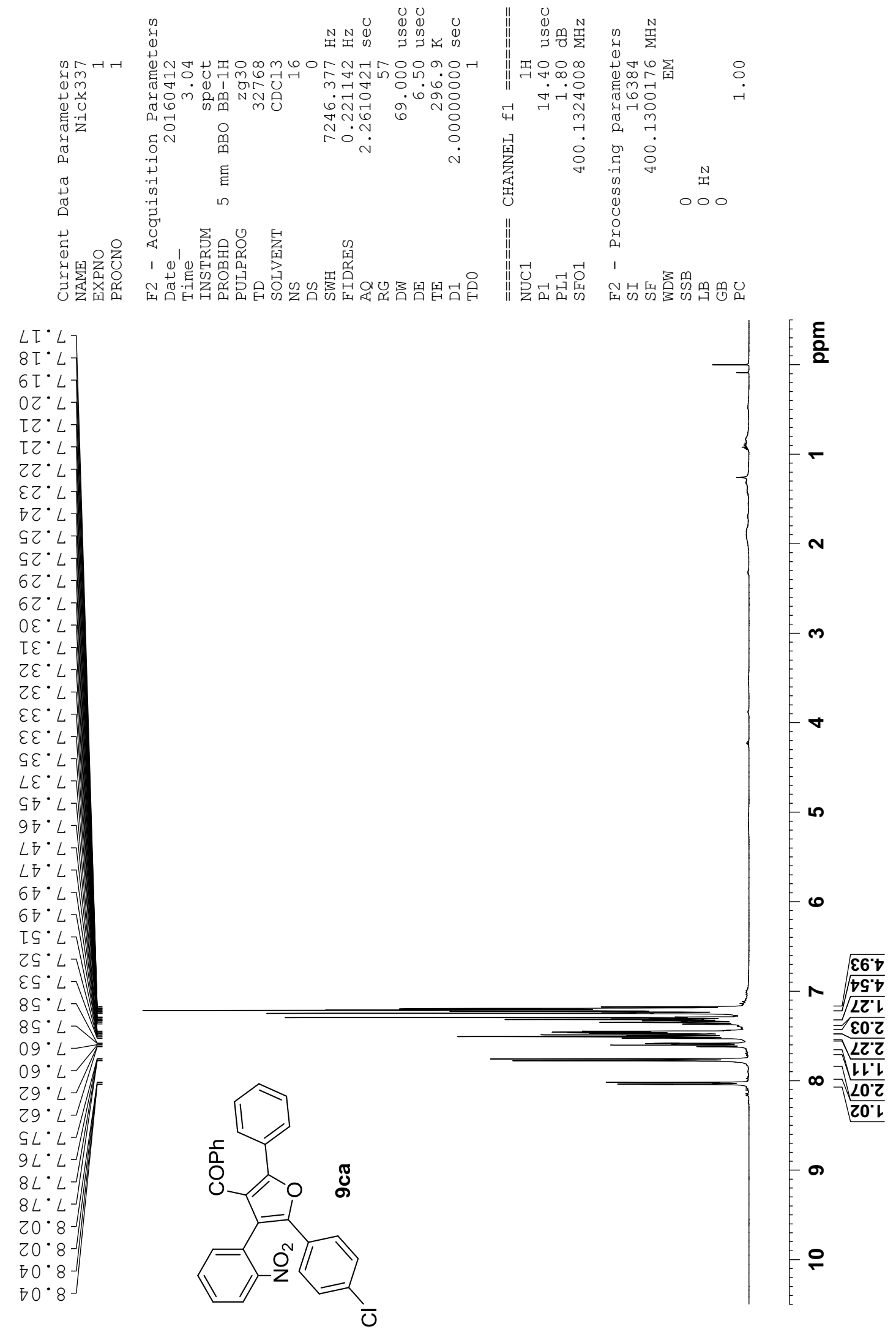

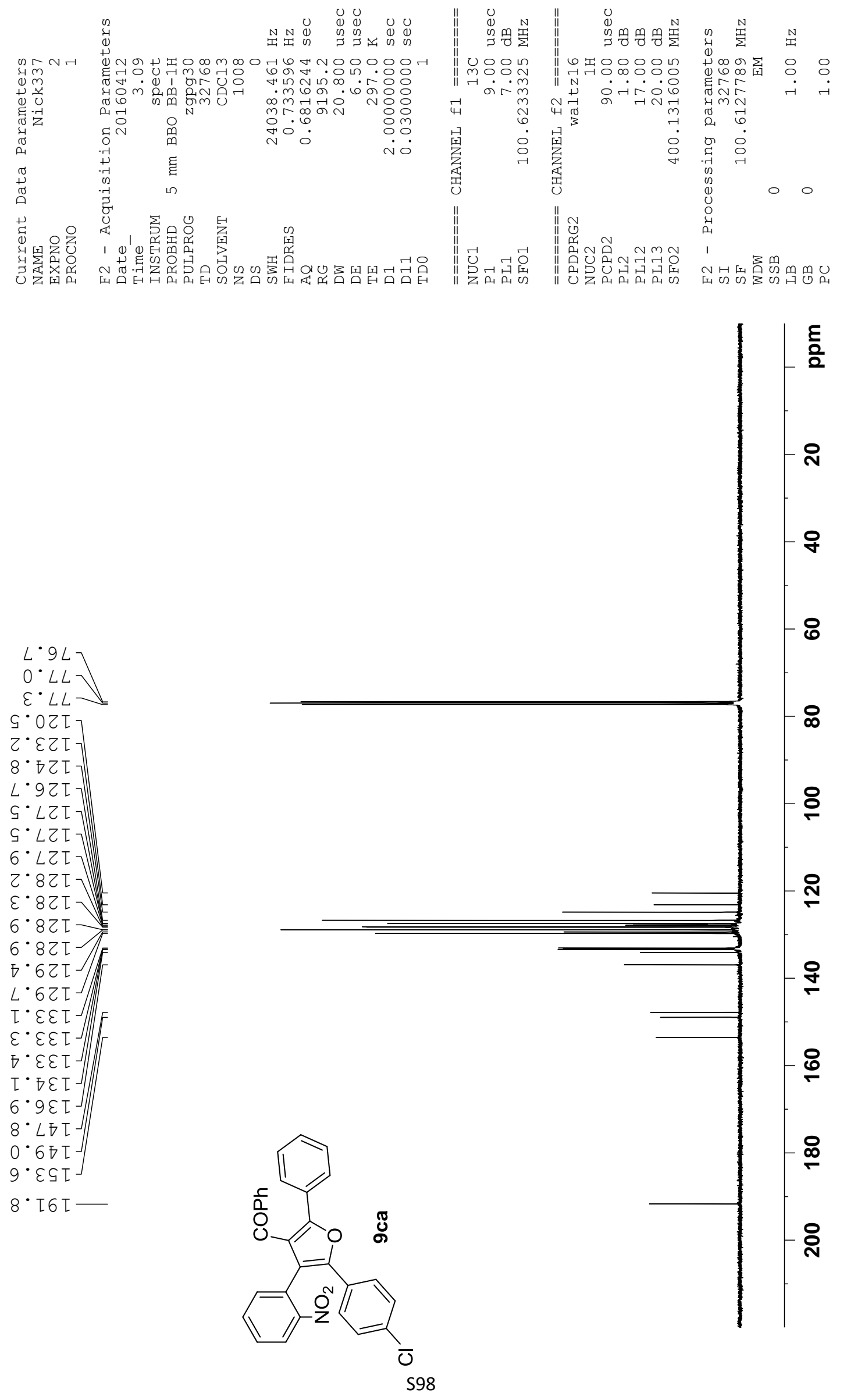

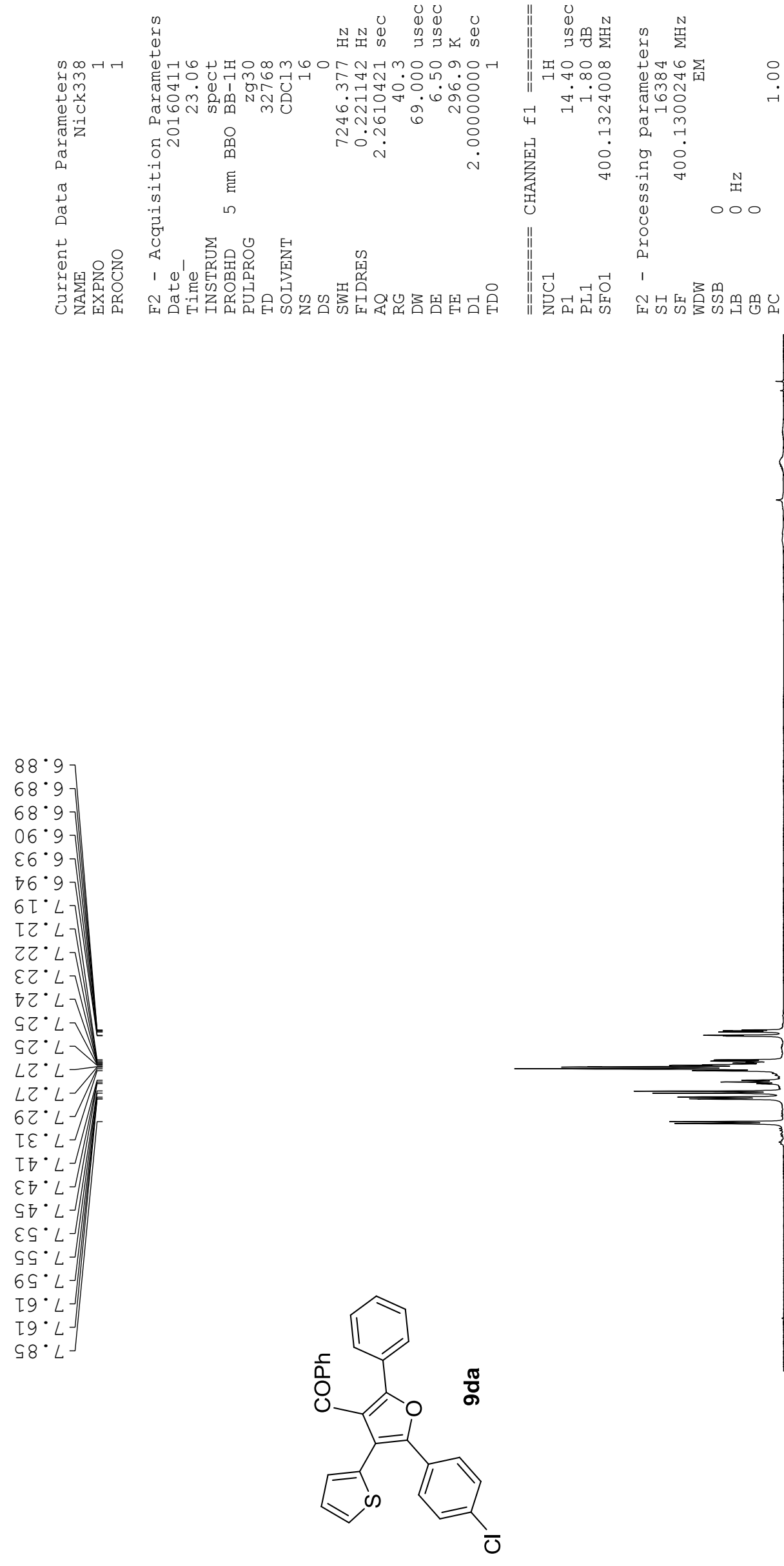

흥

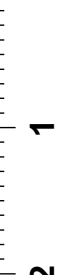

m

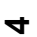

เ

6

$\sqrt{00^{\circ} L}$

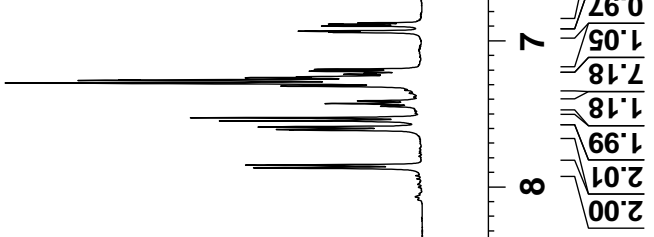



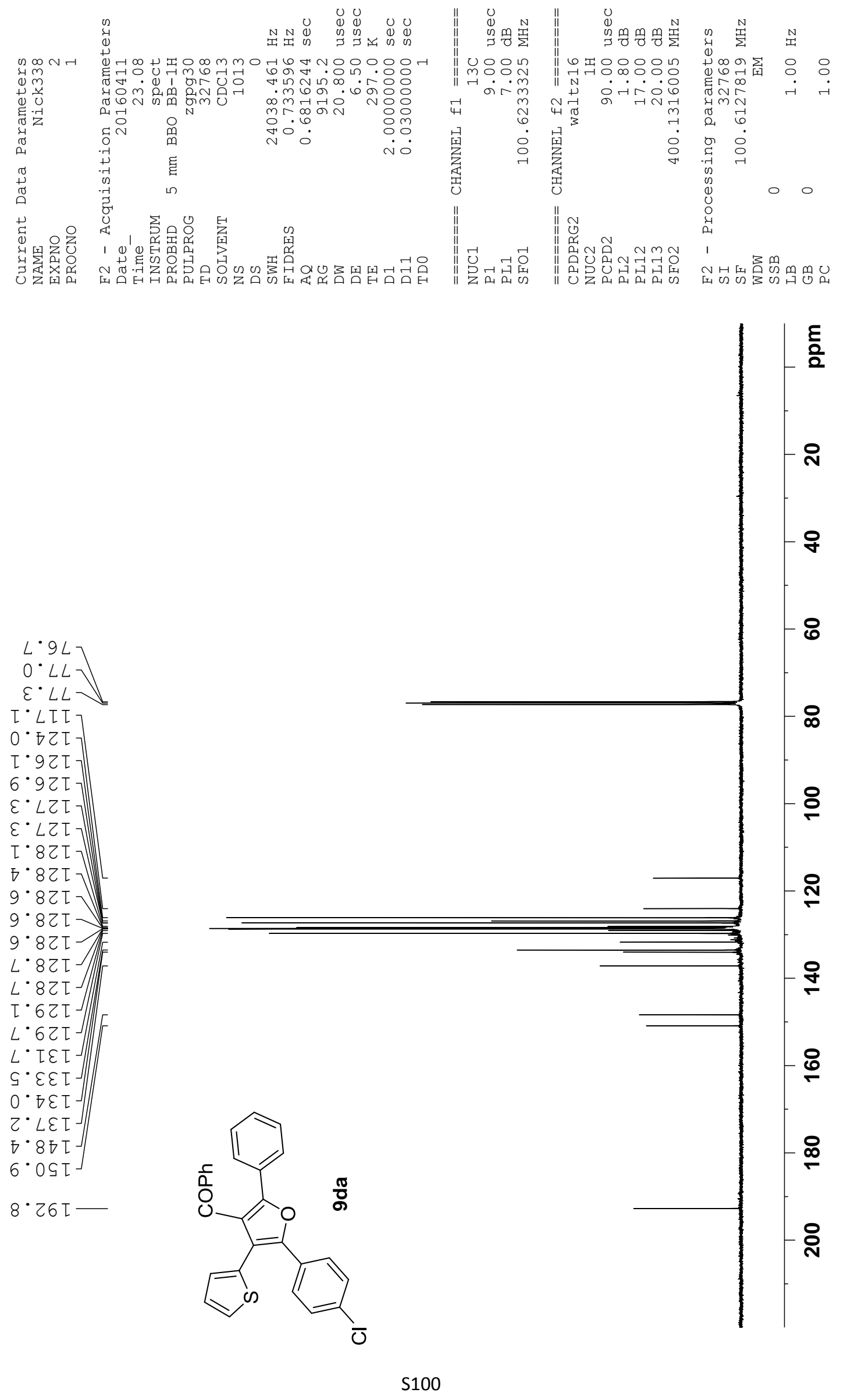

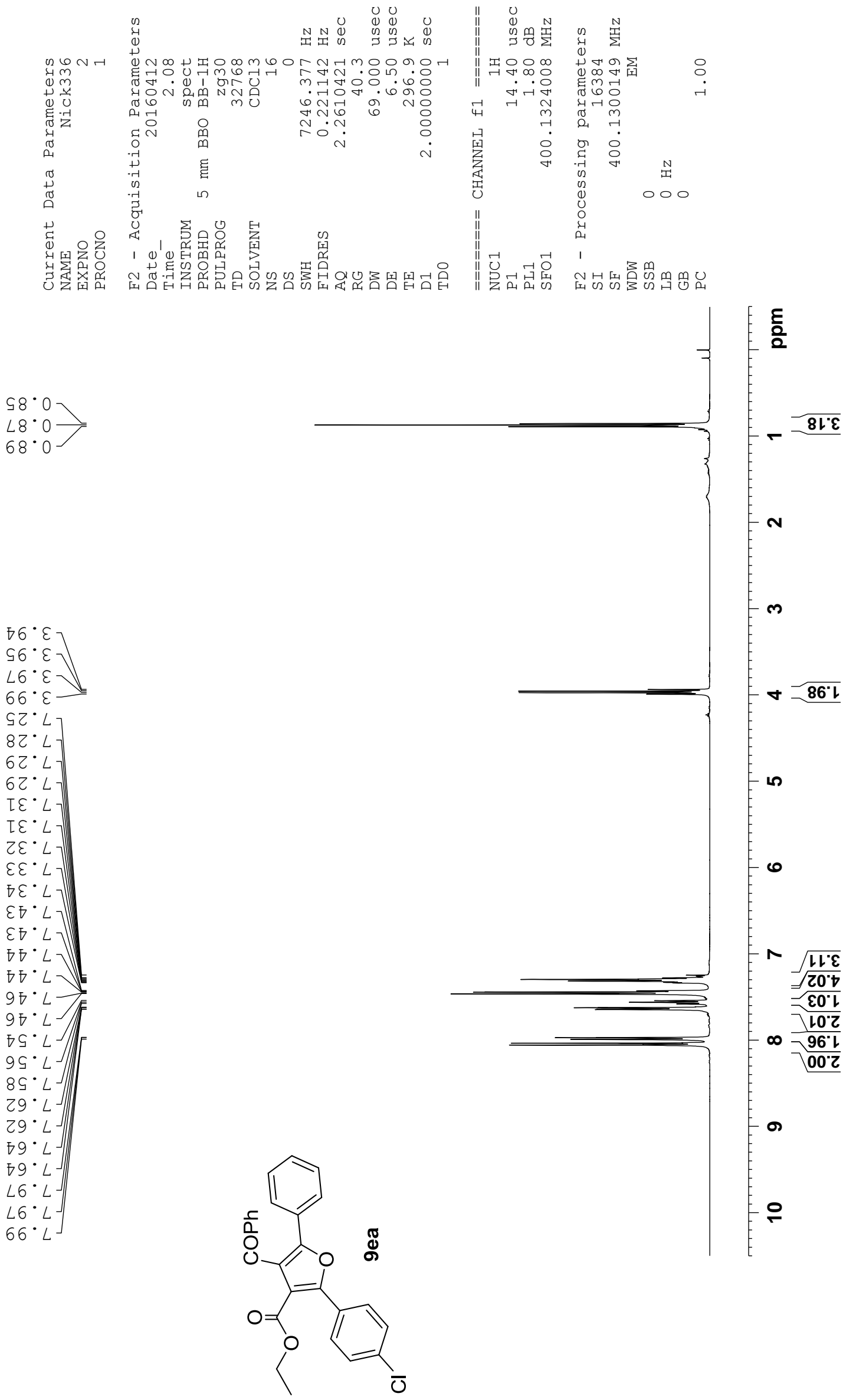


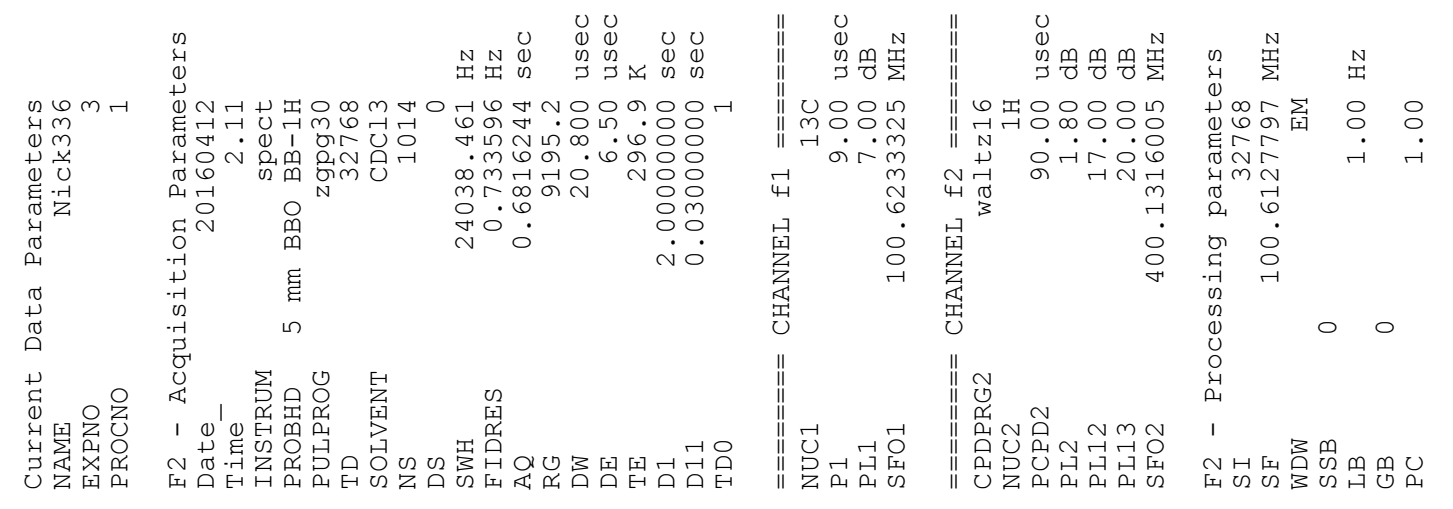

$$
\tau \cdot \varepsilon \tau
$$
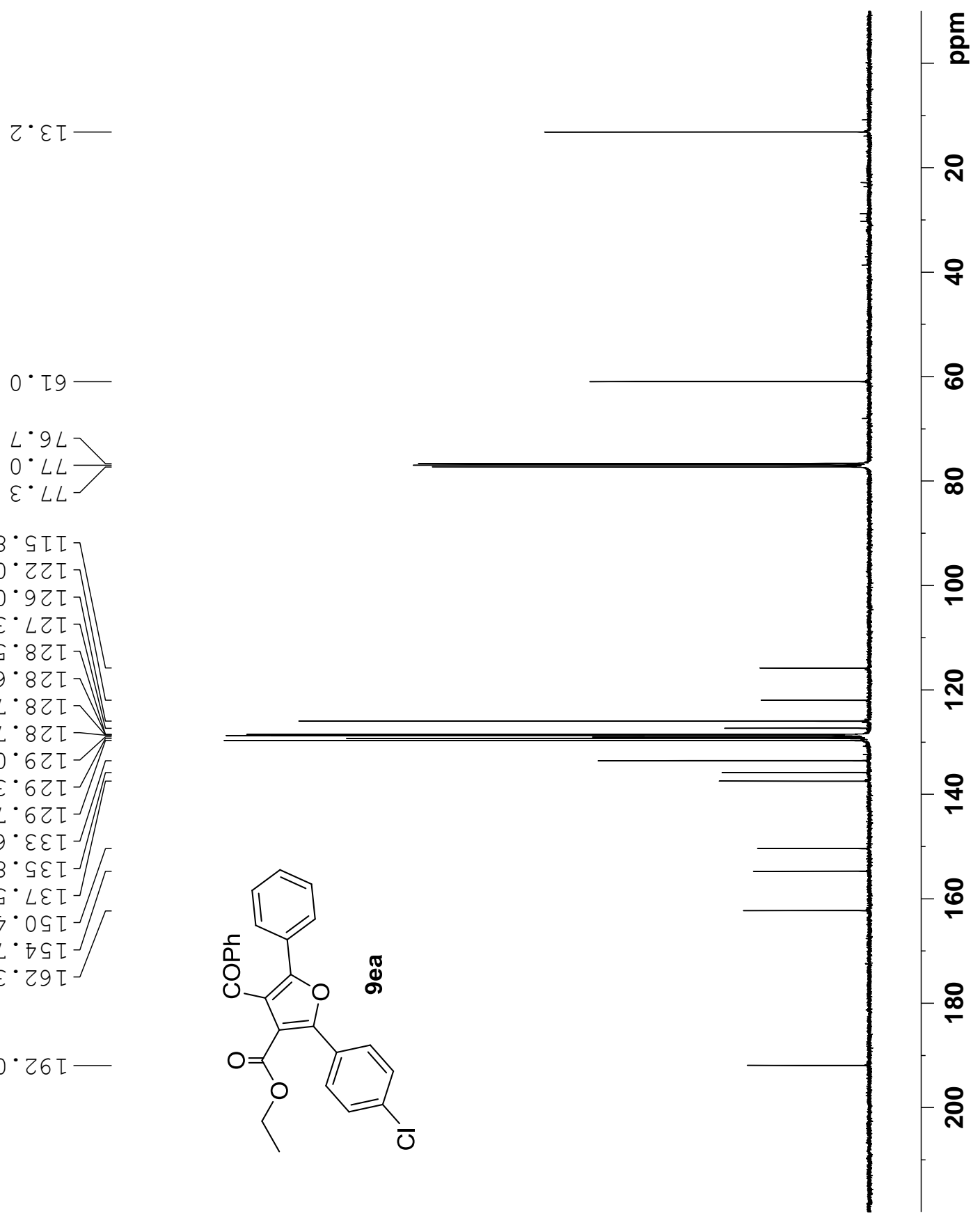


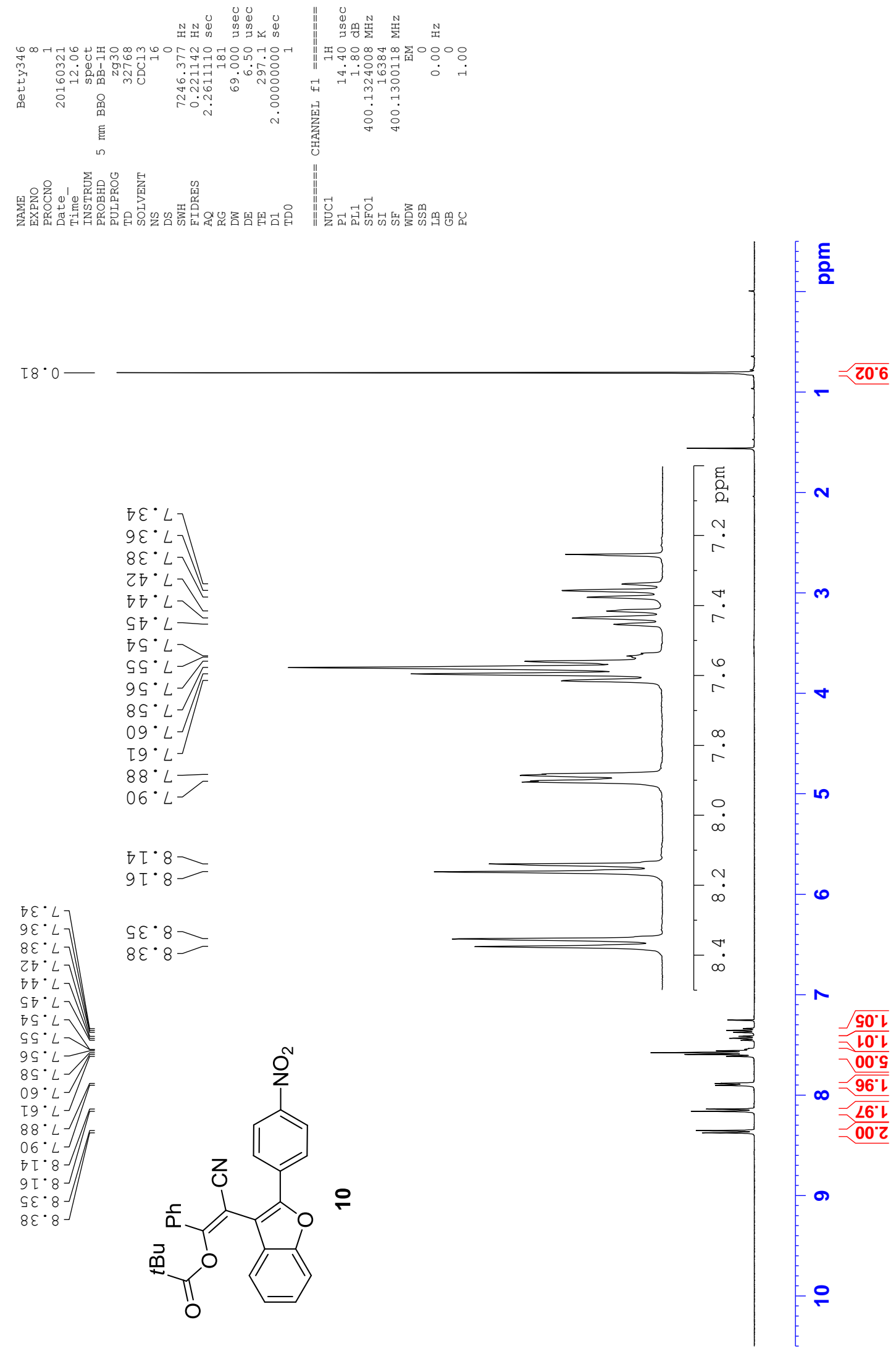




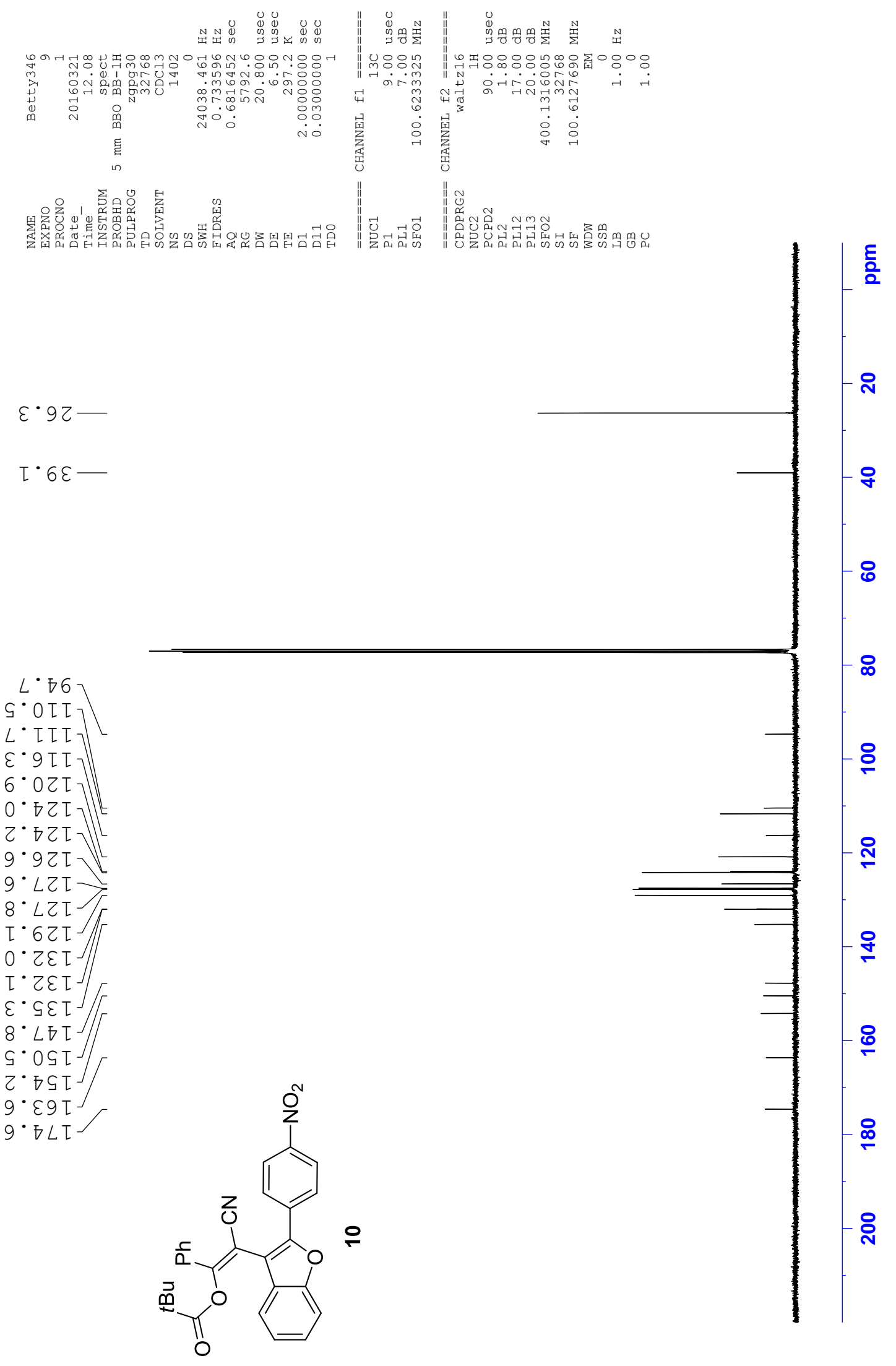



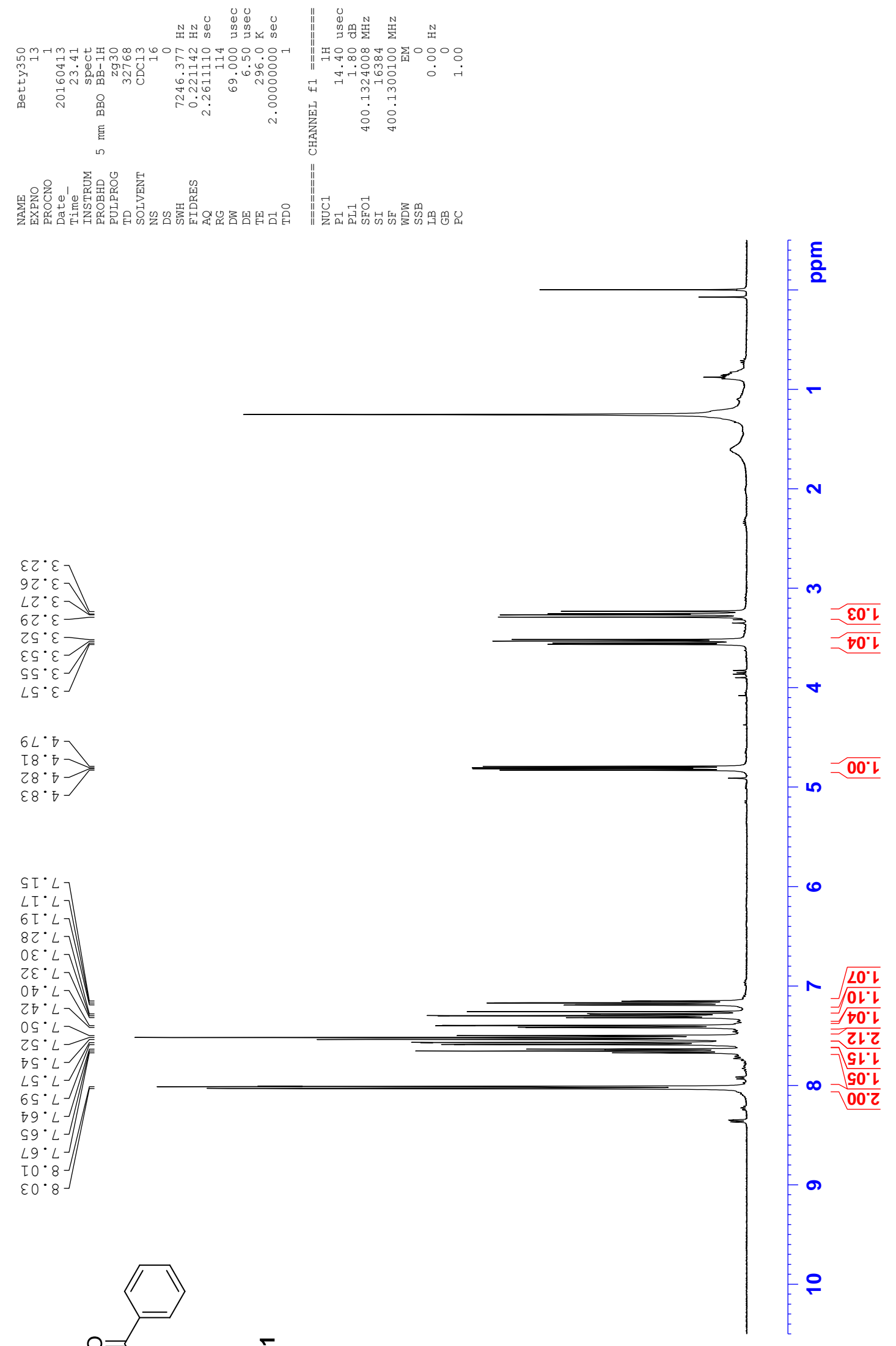<smiles>O=C(c1ccccc1)C([Hg])Cc1ccccc1[18F]</smiles> 

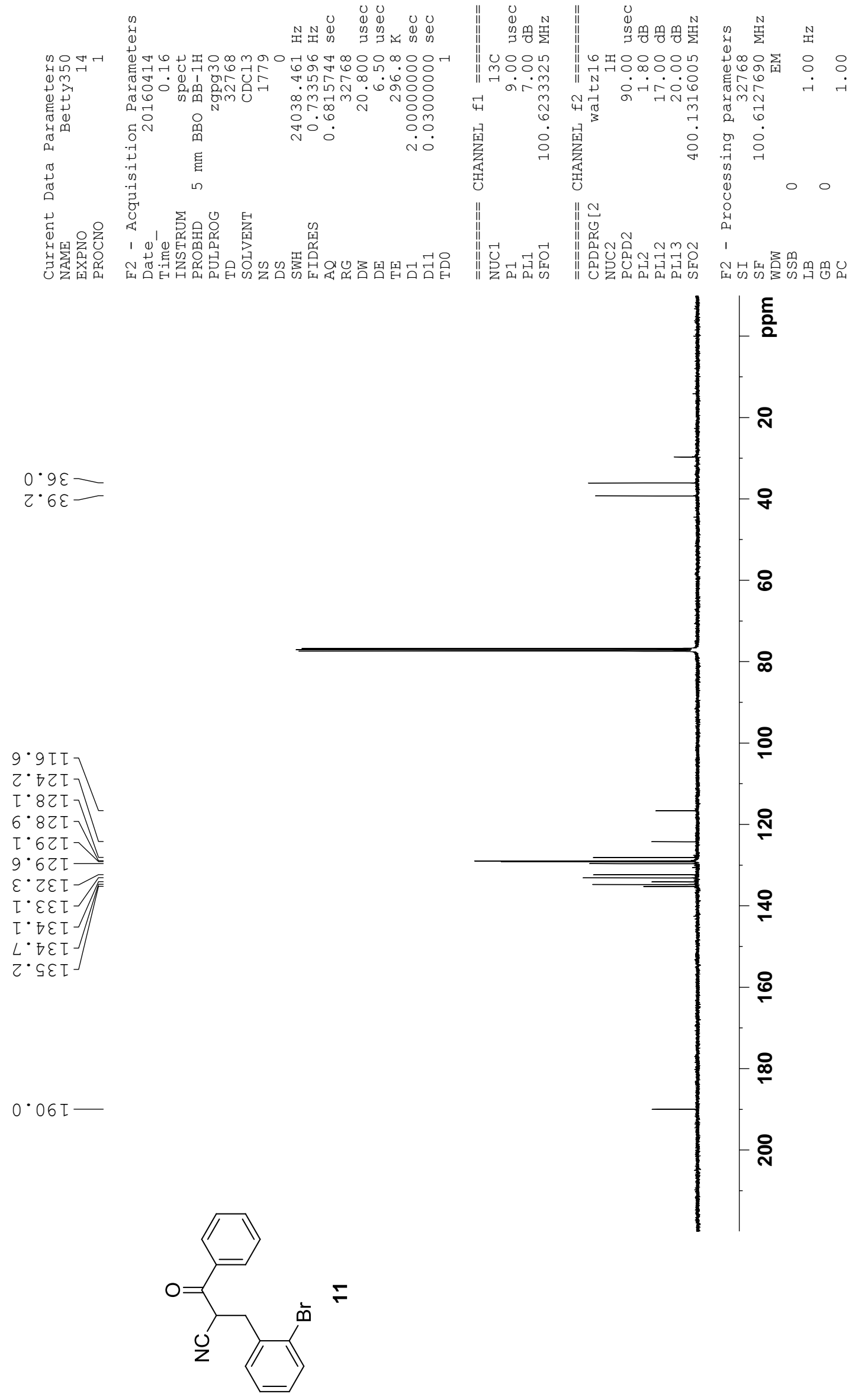